in hepatic fibrosis despite reversal of hyperbilirubinemia with Omegaven ${ }^{\circ}$. Detailed pathologic findings of whole explanted native livers in children who have undergone a combined liver-intestine transplant on Omegaven therapy have not been reported.

Design: Explanted whole native livers from seven patients who have received Omegaven $^{\circ}$ emulsion as parenteral nutrition therapy in the pretransplant period were evaluated. Histological features including degree of inflammation, fibrosis, steatosis, cholestasis and portal bile duct proliferation were semiquantitatively scored according to established criteria.

Results: The pathological features of the seven native liver explants are summarized in the following table.

\begin{tabular}{|l||l||l|l|l|l|l||l|l||}
\hline Features & Pt. 1 & Pt. 2 & Pt. 3 & Pt. 4 & Pt. 5 & Pt. 6 & Pt. 7 \\
\hline Protal inflammation(0-4) & 1 & 1 & 1 & 1 & 1 & 2 & 1 \\
\hline Bile Duct Proliferation(0-3) & 1 & 3 & 1 & 1 & 1 & 3 & 1 \\
\hline Interface Hepatitis(Yes/No) & No & No & No & No & No & Yes & No \\
\hline Steatosis(0-3) & 1 & 1 & 1 & 0 & 1 & 0 & 0 \\
\hline Cholestasis(0-3) & 1 & 3 & 2 & 0 & 0 & 2 & 0 \\
\hline Lobular Hepatitis(Yes/No) & No & No & No & No & No & No & No \\
\hline Clear Cell Change(Yes/No) & Yes & Yes & No & No & No & Yes & No \\
\hline Eibrosis Stage(0-4) & 3 & 3 & 3 & 4 & 4 & 4 & 3 \\
\hline
\end{tabular}

All patients had resolution of hyperbilirubinemia while on Omegaven ${ }^{\circ}$ therapy. Six of the seven patients $(86 \%)$ showed minimal active inflammation. All cases showed bridging fibrosis and 3 of them $(42.9 \%$ ) had established cirrhosis (stage 4 ).

Conclusions: Pediatric intestinal transplant recipients who received Omegaven ${ }^{\odot}$ emulsion therapy showed minimal to mild inflammation, mild cholestasis and yet advanced fibrosis in their explants. The reason for this discordance is unclear as a chronic inflammatory state has generally been considered a precursor to advanced

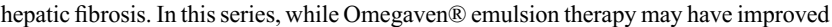
degree of inflammation and cholestasis, progression to severe fibrosis and cirrhosis was not altered.

1936 Clinical Co-Morbidities, Ablative Site Placental Calcification, and Vascular Remodeling in Twin-to-Twin Transfusion Syndrome with and without Selective Fetoscopic Laser Photocoagulation (SFLP)

SE Starnes, P Fitchev, C Thorpe, E Vlastos, S Mehra, M Cornwell, SE Crawford. Saint Louis University School of Medicine, St. Louis, MO.

Background: Twin-to-twin transfusion syndrome (TTTS), imbalanced shunting of blood from one twin to the other via placental vascular anastomoses, complicates approximately 10 to $15 \%$ of monochorionic pregnancies and carries a high risk of fetal mortality. The current treatment of choice for TTTS is selective fetoscopic laser photocoagulation (SFLP) and ablation of the vascular anastomoses. Recurrence of TTTS after SFLP occurs in up to $16 \%$ of cases and is associated with an adverse outcome. Previous studies of placental pathology in TTTS cases have primarily focused on angioarchitecture in placentas not treated by SFLP with scant evaluation of other microscopic findings.

Design: Charts of 9 cases of twin pregnancies affected by TTTS were reviewed; 6 underwent SFLP and 3 had no intervention. All cases were assessed by ex vivo vascular injection studies with gross and microscopic evaluation. Sections were taken at SFLP sites, remote from sites, and of any grossly abnormal regions. Studies including immunohistochemistry were performed to highlight the vasculature (CD31, smooth muscle actin, endothelin) and to confirm calcium deposition.

Results: Of TTTS cases ( $\mathrm{n}=9$ ), mean maternal age was 24 yrs (range 20-32 yrs). For this young age group, there was a high rate of co-morbidities including obesity, diabetes, biliary disease, smoking, and hematopoietic abnormalities. Fetal demise of one twin occurred in $67 \%(2 / 3)$ of the untreated group, but in those with SFLP, the rate was only $33 \%(2 / 6)$. Vascular studies showed residual anastomoses in one case. There was abnormal umbilical cord insertion in $44 \%$ (4/9). Histologic analysis revealed vascular pathology including intravascular calcifications in $67 \%(6 / 9)$, two of which had no SFLP. In 6/7 cases with SFLP treatment, fine concentric calcifications surrounded areas of villous fibrosis underlying SFLP sites and in 3/7, there was a wedge-shaped infarct subjacent to the SFLP site.

Conclusions: This cohort of women with gestations complicated by TTTS was young, with a high number of co-morbidities, especially metabolic (30\%). There was accelerated mineralization of choriovillous vasculature in TTTS placentas regardless of SFLP status, suggesting a new disease association. The wedge-shaped infarcts associated with SFLP sites indicate that superficial chorionic intervention can significantly alter subjacent placental perfusion.

\section{Pulmonary Pathology}

1937 ALK Rearrangement Detection in NSCLC: Comparison between Fluorescent In Situ Hybridization, Immunohistochemistry, and MassARRAY Based Method

G Ali, A Proietti, S Pelliccioni, C Niccoli, C Lupi, E Sensi, R Giannini, N Borrelli, $M$ Menghi, A Chella, A Ribechini, F Cappuzzo, F Melfi, M Lucchi, A Mussi, G Fontanini. Unit of Pathological Anatomy, Azienda Ospedaliera Universitaria Pisana, Pisa, Italy; University of Pisa, Pisa, Italy; Diatech Pharmacogenetics, Pisa, Italy; Unit of Pneumology, Azienda Ospedaliera Universitaria Pisana, Pisa, Italy; Endoscopic Section of Pneumology, Azienda Ospedaliera Universitaria Pisana, Pisa, Italy; Istituto Toscano Tumori, Ospedale Civile, Livorno, Italy; Unit of Thoracic Surgery, Azienda Ospedaliera Universitaria Pisana, Pisa, Italy.

Background: $E M L 4-A L K$ translocation has been described in a subset of patients with non-small cell lung cancer (NSCLC) and has been shown to have oncogenic activity. Fluorescent in situ hybridization (FISH) is used to detect $A L K$-positive NSCLC, but it is expensive, time-consuming and difficult for routine application. For these reasons, we evaluated the potential role of immunohistochemistry (IHC) as a screening tool to identify candidate cases for FISH analysis and for ALK inhibitor therapy in NSCLC. To verify the mRNA expression of EML4-ALK, we used a MassARRAY method in a small subset of patients.

Design: We performed FISH and IHC for $A L K$ and mutational analysis for $E G F R$ and $K$-ras in 523 NSCLC specimens. We conducted IHC analysis with the monoclonal antibody D5F3 and a highly sensitive detection system. We also performed a MassARRAY-based analysis in a small subset of 11 samples to detect EML4-ALK rearrangement

Results: Of the 523 NSCLC specimens, 20 (3.8\%) were positive for $A L K$ rearrangement by FISH analysis. EGFR and Kras mutations were identified in $70(13.4 \%)$ and 124 (23.7\%) out of 523 tumor samples, respectively. $A L K$ rearrangement, EGFR and Kras mutation were mutually exclusive. Out of 523 analyzed tumor samples, $18(3.4 \%)$ were ALK positive by IHC. 18 samples had concordant IHC and FISH results, and 2 ALK FISH-positive cases failed to show ALK protein expression. In these two discrepant cases, MassARRAY confirmed the absence of EML4-ALK expression.

Conclusions: In conclusion, our results show that IHC may be a useful technique for selecting NSCLC cases to undergo $A L K$ FISH analysis.

1938 Necrosis and Nuclear Grade Are Predictors of Overall Survival in Epithelioid Malignant Mesotheliomas (EMM)

$V$ Ananthanarayanan, S McGregor, Q Arif, D Hadi, $M$ Alikhan, $W$ Vigneswaran, $T$ Krausz, AN Husain. University of Chicago, Chicago, IL.

Background: Mesotheliomas with epithelioid histology are considered to have a better prognosis than biphasic or sarcomatoid mesotheliomas. A recently published article demonstrated the prognostic importance of nuclear grading in predicting survival in patients with epithelioid diffuse malignant pleural mesothelioma (Kadota K etal. Mod Path, 2012, 25, 260-71). The current study was undertaken to determine the usefulness of this grading system.

Design: We examined all resected/ debulked cases of pleural epithelioid malignant mesothelioma (EMM) from 2006 to 2010 after appropriate institutional review board approval. H\&E slides were reviewed and nuclear grade was computed combining nuclear pleomorphism and mitotic rates into a semi-quantitative score of grades I-III using Kadota et al's grading system. The presence or absence of any necrosis and the predominant patterns of growth were also evaluated. Overall survival (OS) was used as the primary end point for all data analysis. Data were examined using Pearson's Chi-square test, proportional hazards regression and log rank tests within STATA 11® (StataCorp, Texas).

Results: A total of 33 patients (6:1 male: female ratio) with 27 low grade (12 Grade I and 15 Grade II) and 6 high grade (Grade III) EMMs were analyzed. Grade was significantly associated with necrosis $\left(\mathrm{pX}^{2}=0.032\right)$. A total of 9 deaths were noted among 26 patients with a mean follow up duration of 40 months (range 6-86). Univariate analyses showed that necrosis impacted $\mathrm{OS}\left(\mathrm{p}_{\text {log-rank }}=0.01\right.$, figure $\left.1 \mathrm{~b}\right)$. A predominant solid pattern was marginally associated with $\operatorname{OS}\left(\mathrm{p}_{\text {. }}=0.06\right)$ while age did not impact outcome $(\mathrm{p}=\mathrm{n} . \mathrm{s}$.). Furthermore, nuclear grade as a two-tier $(\mathrm{HR}=8.50,95 \% \mathrm{CI}=1.40$, 51.50 , figure $1 \mathrm{a})$ as well as three-tier system $(\mathrm{HR}=3.77 ; 95 \% \mathrm{CI}=1.17-12.17)$ impacted outcome significantly.

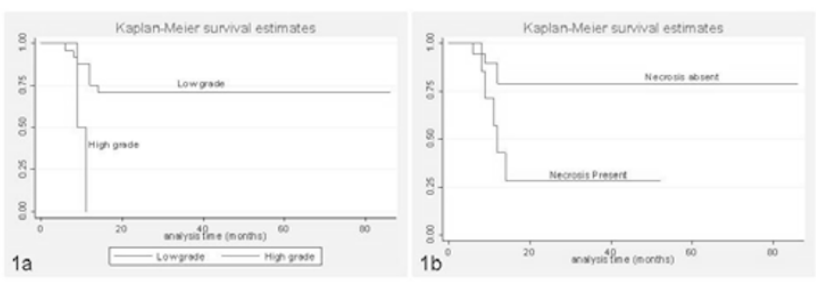

Conclusions: This study performed on a limited sample size emphasizes the importance of examining nuclear grade, patterns of growth and presence or absence of necrosis while evaluating resection specimens of EMM. Multivariate analyses of a larger dataset will clarify the impact of these findings.

1939 Thoracic Epithelioid Vascular Tumors: Prognostic Factors and Usefulness of WWTR1-CAMTA1 Fusion in the Distinction of Epithelioid Hemangioendotheliomas and Angiosarcomas

TA Anderson, WD Travis, CR Antonescu. Memorial Sloan-Kettering Cancer Center, New York, NY.

Background: Epithelioid vascular tumors are a heterogeneous group of tumors that encompass a morphologic spectrum between low and intermediate grade (G1 and G2) epithelioid hemangioendotheliomas (EHE) and high grade (G3) epithelioid angiosarcomas (EA). A WWTR1-CAMTA1 fusion gene has been previously reported in conventional EHEs of various sites; however, only few intrathoracic cases have been studied.

Design: 49 thoracic epithelioid vascular tumors were obtained from the case files at our institution and personal consultations. The clinical course, histologic features and immunohistochemistry (IHC) were reviewed. Fluorescence in-situ hybridization (FISH) analysis for WWTR1 and CAMTA1 gene rearrangements were performed on 30 cases. Results: There were $36 \mathrm{M}$ and 13F with EHE: G1 $(\mathrm{n}=12)$ and $\mathrm{G} 2(\mathrm{n}=24)$ and EA: G3 $(\mathrm{n}=13)$. Presentation was exclusively thoracic $(\mathrm{n}=33)$, multiorgan including lung $(\mathrm{n}=14)$ and metastatic to lung from extrathoracic sites $(n=2)$. The thoracic tumors presented in the pleura $(n=19)$, lung $(n=23)$, mediastinum $(n=5)$, and superior vena cava $(n=1)$. CAMATA1 rearrangements were found in 16/17 (94\%) G2, 5/9 (56\%) G1 and 0/4 
G3 tumors ( $<<0.001)$. WWTR1 rearrangements were found in 16/17 $(94 \%) \mathrm{G} 2,5 / 9$ $(56 \%) \mathrm{G} 1$, and $1 / 4 \mathrm{G} 3(25 \%)(\mathrm{p}<0.005)$. Within EHE, both rearrangements were found significantly more frequent in $\mathrm{G} 2$ than G1 EHE $(\mathrm{p}=0.034)$. IHC was positive for ERG $8 / 10(80 \%)$, CD31 42/45 (93\%) and CD34 31/46 (67\%). Death occurred in 11/12 (92\%) EA compared to $14 / 28(50 \%)$ EHE with followup $(\mathrm{p}=0.015) .4$ year survival was significantly worse for EA $(11 \%)$ compared to EHE $(33 \%, p=0.038)$. For lung cases, 4 year survival was worse for those with pleural thickening $(0 \%)$ compared to those without $(50 \%, \mathrm{p}=0.049)$

Conclusions: Epithelioid vascular tumors of the thoracic cavity can be classified as low to intermediate grade EHE and high grade EA. This is supported by our survival data as well as the presence of WWTR1-CAMTA1 fusion in most conventional EHE cases with a rare finding of WWTR1 in EA. In the presence of necrosis, nuclear pleomorphism and increased mitotic activity in a tumor that lacks vasoformative features, the differential diagnosis between an EA and a malignant EHE remains arbitrary based on morphology alone. Demonstration of WWTR1 or CAMTA1 rearrangements offers an objective criterion that can help establish the diagnosis of EHE and make the distinction from EA. CD31 and ERG appear to be the most useful vascular IHC markers.

1940 Liquid-Chromatography Mass Spectrometry (LC-MS) Analysis of Pulmonary Nodular Light Chain Deposition Disease (NLCDD) - An Entity Associated with Sjogren Syndrome (SS) and Marginal Zone Lymphoma (MZL)

AV Arrossi, M Merzianu, C Farver, C Yuan, SH Wang, MO Nakashima, CV Cotta. Cleveland Clinic, Cleveland, OH; Roswell Park Cancer Institute, Buffalo, NY.

Background: NLCDD is a rare disease, with no systemic dissemination. It is characterized by deposits of amorphous material which on routine examination are identical to amyloid. The very few studies investigating NLCDD used electron microscopy (EM) to differentiate it from amyloid, but the composition of the deposits was examined by immunohistologic techniques, currently considered suboptimal. LC-MS is considered to be the gold standard for analysis of amyloid, but there is no data investigating the composition of NLCDD deposits by LC-MS. Moreover, as most cases were single reports or very small series, information regarding the association of NLCDD with hematolymphoid or autoimmune disorders is limited.

Design: 7 cases of NLCDD were examined by routine histologic techniques and immunohistochemistry. Clinical and laboratory correlations were obtained from electronic medical records. Laser microdissection of formalin-fixed paraffin-embedded tissue from 6 cases was digested with trypsin, followed by LC-MS analysis on a Thermo Scientific Q Exactive mass spectrometer. For peptide identification an IPI human protein database was used. The cases were classified as NLCDD only when the constant components of amyloid-Serum protein P, Apolipoprotein (Apo) AI, Apo AIV and Apo $\mathrm{E}$ were not identified. In one case diagnosis was confirmed by EM. Results: See table.

\begin{tabular}{|l|l||l|l|l||}
\hline Diagnosis & M-protein & Biopsy Site & Congo Red & LC-MS \\
\hline MZL \& SS & IgM $\kappa$ & Jugular LN & Negative & $\kappa$ \\
\hline LCDD only & None & Lung & Negative & N/A \\
\hline MZL \& SS & On polyclonal background & Thymus \& lung & Negative & No light chain \\
\hline MZL & IgM $\kappa$ & Lung & Negative & $\kappa$ \\
\hline MZL & None & Lung & Negative & $\lambda$ \\
\hline LPL/PCN & IgG $\kappa$ and IgM $\kappa$ & Lung & Negative & $\kappa$ \\
\hline SS & None & Lung & Negative & $\kappa$ \\
\hline
\end{tabular}

LPL- lymphoplasmacytic lymphoma; PCN- plasma cell neoplasm

Conclusions: This is the largest study of NLCDD and the only one using LC-MS. 6 (86\%) of cases were associated with MZL/LPL and/or SS. In contrast to amyloidosis, in NLCDD the most often encountered light chains are kappa. With one exception, there was no systemic involvement and renal function was preserved. In cases with lymph node (LN) or thymus involvement lung nodules were described by imaging studies, but no other lesions were detected. Therapy received varied from excision, local radiation to systemic chemotherapy. One patient died, but all others are alive, at intervals varying 1-10 years from diagnosis. NLCDD is a distinct entity from systemic LCDD, but has numerous morphologic and clinical features overlapping nodular pulmonary amyloidosis.

1941 Chromosomal Rearrangements and Copy Number Abnormalities of TP63 Correlate with p63 Protein Expression in Lung Adenocarcinoma M-C Aubry, A Roden, SJMurphy, G Vasmatzis, SH Johnson, RA Knudson, RP Ketterling, AL Feldman. Mayo Clinic, Rochester, MN.

Background: The TP 63 gene encodes a member of the p53 family of transcription factors. Although TP53 is a well-known tumor suppressor gene and its role in tumorigenesis has been extensively studied, the role of p63 in tumorigenesis is controversial. A subset of lung adenocarcinomas (ADs) expresses p63 and shows TP63 amplification, although the significance of this finding remains unclear. Using next-generation sequencing, our group recently identified novel chromosomal rearrangements involving TP63 in peripheral T-cell lymphomas, and demonstrated that these rearrangements were associated with a $\mathrm{p} 63+/ \mathrm{p} 40$ - immunohistochemical profile. Since a subset of lung ADs has this immunoprofile, we undertook the current study to examine the presence of TP63 rearrangements.

Design: Laser capture microdissection of frozen tumor and adjacent normal was whole genome amplified in 37 ADs. Next generation sequencing of mate-pair DNA libraries was used to identify genomic rearrangements of TP63. Confirmatory FISH using a breakapart probe to the TP63 gene region and IHC for p63 and p40 were performed on ADs with TP63 rearrangements identified by mate-pair sequencing. IHC for p63 and $\mathrm{p} 40$ also was performed on 45 additional ADs, and FISH was performed on all ADs with p63 positivity.
Results: TP63 rearrangement was identified in 2 ADs (synchronous lung tumors from 1 patient). The rearrangement resulted in an inversion of $3 \mathrm{q}$ that fused B3GALNT1 to TP63. FISH confirmed the rearrangement in both tumors. IHC staining for $\mathrm{p} 63$ was diffuse ( $>80 \%$ cells + ) and p 40 was negative. Of the 45 additional ADs, $15(33 \%)$ showed p63 expression in $20-60 \%$ of cells; p40 was negative in all cases. No case showed rearrangement of TP63 by FISH. However, extra copies of the intact TP63 locus were seen in all 12 cases, with copy numbers ranging from 3 to 7 . Extra copies of TP63 were seen in the p63+ areas while only 2 copies of TP63 were present in the p63-negative ( 3 of 4 cases scored).

Conclusions: We have identified a novel chromosomal rearrangement involving TP63 in a p63+/p40- lung AD. Breakapart FISH testing can be used to diagnose this finding. IHC for $\mathrm{p} 63$ was not specific for this rearrangement. Additional copies of the intact TP63 locus also were a common finding and correlated with IHC positivity for p63. FISH results suggest that partial positivity for p63 by IHC may reflect clonal heterogeneity within lung $\mathrm{ADs}$.

1942 Expression of p63 and p40 in Small Cell Carcinoma of the Lung R Bhatnagar, R Sharma, PB Illei. Johns Hopkins Medical Institutions, Baltimore, MD. Background: Morphologic distinction between small cell carcinoma and squamous cell carcinoma can be challenging especially on small biopsy material, including fine needle core biopsies, transbronchial biopsies and on cell blocks of cytologic material. Immunohistochemistry using markers of neuroendocrine differentiation (synaptophysin, chromogranin and CD56) are helpful but can be all negative in a subset small cell carcinoma, particularly in biopsy material. Immunohistochemistry for $\mathrm{p} 63$ has been commonly used as a marker of squamous differentiation, however, p63 expression has been described in a subset of lung adenocarcinoma, and in rare cases of small cell lung carcinoma. We have evaluated the role of a novel p40 $(\Delta \mathrm{p} 63)$ monoclonal antibody in distinguishing small cell lung carcinoma from poorly differentiated squamous cell carcinoma.

Design: Immunohistochemistry using mouse monoclonal antibodies for p63 (clone 4A4) and $\mathrm{p} 40$ (clone $\mathrm{BC} 28$ ) were performed on commercially available tissue microarrays of 69 cases of small cell lung carcinoma. The staining was performed on a fully automated immunostainer using polymer based detection. We have also stained 7 cases of poorly differentiated adenocarcinomas of the lung that have previously been focally positive for $\mathrm{p} 40$ using a rabbit polyclonal antibody.

Results: Overall, positive p63 staining was observed in 13 of the $69(19 \%)$ small cell carcinomas ranging from strong diffuse staining to scattered positive cells (table 1.). Moderate to strong tumor staining in more than 5\% of tumor cells was observed in 6 cases $(9 \%)$. In contrast, all 69 cases were negative for $\mathrm{p} 40$ using the monoclonal antibody (clone BC28). The 7 cases of poorly differentiated adenocarcinomas showed the same focal staining (with similar intensity) for both p40 antibodies.

p63 and p40 staining in small cell carcinoma of lung $(n=69)$

\begin{tabular}{|l|l|l||}
\hline \hline & $\mathrm{p} 63$ & $\mathrm{p} 40$ \\
\hline Strong diffuse staining & 1 & 0 \\
\hline Moderate staining in $>50 \%$ of tumor & 3 & 0 \\
\hline Moderate staining in $<50 \%$ of tumor & 2 & 0 \\
\hline Rare strongly positive cells & 7 & 0 \\
\hline
\end{tabular}

Conclusions: P63 positivity can be seen in a subset of small cell carcinoma, while no $\mathrm{p} 40(\Delta \mathrm{p} 63)$ staining was observed in the same cohort of tumors. Immunohistochemistry for p63 alone should not be used as single marker to exclude squamous differentiation in small cell carcinoma of the lung. The monoclonal p40 antibody showed similar staining characteristics to a commonly used polyclonal antibody in a selected rare cohort of poorly differentiated adenocarcinoma.

\section{Detection of FGFR3 Mutations in Lung Squamous Cell} Carcinoma Using a SNaPshot Assay

CC Black, F de Abreu, TL Gallagher, JR Rigas, KH Dragnev, GJ Tsongalis. DartmouthHitchcock Medical Center, Lebanon, NH.

Background: Lung squamous cell carcinoma (SCC) is one of the common human cancers with significant morbidity and mortality rates. There are no approved targeted therapies for this tumor type and thus molecular molecular alterations (amplifications or mutations) of SCC continues to be an area of intense research. The fibroblast growth factor receptor (FGFR) tyrosine kinase family has been shown to be one of the most frequently altered kinases in SCC and mutations in these genes are known to occur in various cancer types. FGFR3 is most commonly mutated in urothelial cancers with lower mutation rates reported in SCC. Tumors harboring FGFR3 mutations may be more sensitive to FGFR and multikinase inhibitors. In this study, we describe a SNaPshot assay for the detection of nine commonly reported mutations in the FGFR3 gene and their prevalence in SCC

Design: Twenty-five archived SCC tissue blocks were selected after review by a pathologist. Ten unstained sections were cut from each block for DNA extraction. FGFR3 mutation analysis was performed using a SNaPshot assay consisting of two panels of mutations. Panel 1 included S373C, G372C, Y375C, K652E/Q and G382R while panel 2 consisted of K652T/M, A393E, S249C and R248C. After PCR amplification, all products were analyzed on an AB3500 for fragment sizing.

Results: DNA extracted from all 25 cases was suitable for analysis. Twenty percent $(5 / 25)$ of the SCC cases showed FGFR3 mutations. Three of the SCCs had the G382R mutation in exon 10, one had the A393E mutation in exon 10 and one had the R248C mutation in exon 7 of the FGFR3 gene. The remaining 20 cases were wildtype for the alleles tested.

Conclusions: FGFR3 mutations may be a common occurrence in SCC and these findings could have clinical implications for future therapeutic options. The SNaPshot assay described here is robust and capable of quickly identifying the most common mutations described in FGFR3. 
1944 Pulmonary Invasive Mucinous Adenocarcinoma - A Clinicopathologic Study with Survival Analysis

JM Boland, JA Wampfler, P Yang, ES Yi. Mayo Clinic, Rochester, MN.

Background: Invasive mucinous adenocarcinoma (IMA) is recognized as a variant of adenocarcinoma (ADCA) in the new international multidisciplinary classification. IMA will replace the term "mucinous bronchioloalveolar carcinoma (BAC)", since they show invasion in most cases and have molecular and clinical characteristics distinct from nonmucinous ADCA. Despite the well-known tendency for multifocal or lobar involvement, clinical outcome of IMA has not been well established compared to non-mucinous adenocarcinoma in situ (formerly non-mucinous BAC) or other invasive ADCAs. Design: Slides from surgically treated lung ADCAs $(n=763)$ with a follow-up for $\geq 5$ years were reviewed by 2 pulmonary pathologists, and comprised 3 non-overlapping cohorts for survival analysis: non-selected cohort of all cases during 2006-7 ("all comers", $n=222$ ), never smokers $(n=266$ ), and former or current smokers (search enriched for "BAC features" in original diagnosis, $n=275$ ). The diagnosis of IMA was agreed by both pathologists. When multiple tumors were present, patients with at least one IMA were included for analysis.

Results: Sixty of 763 patients $(8 \%)$ were found to have IMA. Fifteen of $60(25 \%)$ cases had multifocal IMA, 7 of 60 (12\%) had synchronous or metachronous non-mucinous ADCA, and 1 patient $(2 \%)$ had a synchronous squamous cell carcinoma. Twenty of 60 cases $(33 \%)$ had diffuse lobar involvement or mass-like consolidation $>5 \mathrm{~cm}$, while the remaining 40 cases formed localized masses $<5 \mathrm{~cm}$. When considering cases by cohort, patients with IMA constituted 10 of 222 all comers (4.5\%), 8 of 266 never smokers (3\%), and 42 of 275 BAC enriched smokers $(15 \%)$. In the all comers cohort, IMA was more likely to occur in men ( $80 \%$ of IMA vs. $43 \%$ of non-mucinous ADCA, $\mathrm{p}=0.02$ ), but no differences were observed in regards to gender in the other two cohorts. No difference in stage was found in any cohort. Preliminary analysis shows patients with IMA in the BAC enriched smokers cohort experienced worse progression free (median 3.4 vs. 7 years, $p=0.0095$ ) and overall survival (median 4.4 vs. 9.1 years, $p=0.004$ ) when compared to patients with non-mucinous ADCA. No difference in survival was seen in the other two cohorts.

Conclusions: IMA represent a minority of pulmonary ADCAs (around 4-5\% of nonselected cases) which are frequently large/lobar and multifocal. We suspect that the worse survival of IMA seen in the BAC enriched smokers cohort indicates that IMA have inferior prognosis to non-mucinous ADCAs with high degree of lepidic growth, and supports considering these as an invasive adenocarcinomas with intermediate prognosis.

\section{Frequency of ROS1Rearrangment in Pulmonary Adenocarcinomas} with Signet Ring Cell Features

JM Boland, JS Jang, JA Wampfler, X Wang, MR Erickson-Johnson, AM Oliveira, $P$ Yang, J Jen, ES Yi. Mayo Clinic, Rochester, MN.

Background: Chromosomal rearrangements involving the ROS1 receptor tyrosine kinase gene have recently been described as a rare $(<3 \%)$ driver mutation in pulmonary adenocarcinomas. ROS 1 rearranged tumors $(R O S 1+)$ have shown sensitivity to crizotinib. Patients with $R O S 1+$ tend to be younger and more likely to be never-smokers, similar to those with $A L K$ rearranged tumors $(A L K+)$. While lung adenocarcinomas with signet ring cell features have been associated with $A L K+$, the incidence of $R O S 1+$ in this subgroup of tumors is not well known.

Design: Surgically treated lung adenocarcinomas $(n=764)$ at a single institution were reviewed by 2 pulmonary pathologists. Tumors with signet ring cell features had $>10 \%$ signet ring cells agreed by both pathologists and were TTF1+. ROS1 FISH was performed using 3'ROS1 DNA labeled with SpectrumGreen dUTP (Abbott Molecular/ Vysis Products) and 5'ROS1 DNA labeled in SpectrumOrange dUTP (Abbott Molecular/ Vysis Products) combined as one probe set. The dual-color, single-fusion probe set was applied to individual slides using paraffin FISH protocol.

Results: Fifty-two of 764 cases (7\%) had signet ring cell features, and 47 of these 52 cases had sufficient tissue for FISH testing. Three of 47 signet ring cell cases $(6 \%)$ were $R O S 1+$. All three patients were never smokers, and were negative for other driver mutations (including $A L K, K R A S, E G F R$, and $B R A F$ ). This compares to $13 / 47$ (28\%) of the signet ring cell cases that were $A L K+$. ROSI+ tumors comprised 3/34 (9\%) of signet ring cell tumors that were $A L K$-; $3 / 21$ (14\%) signet ring cell tumors in never smokers; $3 / 13(23 \%)$ signet ring cell tumors that were $A L K$ - in never smokers; $3 / 11$ $(27 \%)$ signet ring cell tumors that were "pan negative" ( $A L K$-, negative for $E G F R$ and $K R A S$ mutations); and $3 / 5(60 \%)$ pan negative signet ring cell tumors in never smokers. Conclusions: The incidence of ROSI+ among lung adenocarcinomas with signet ring cell features was over two-fold higher than the reported incidence in lung adenocarcinomas, though still low at $6 \%$, compared to $28 \%$ of SRC + cases that were $A L K+$. As expected, further enrichment of $R O S 1+$ cases occurred when considering never-smokers and tumors negative for other driver mutations.

\section{Thoracic Peripheral Nerve Sheath Tumors - A Clinicopathological} Study of 72 Cases

JM Boland, TV Colby, AL Folpe. Mayo Clinic, Rochester, MN; Mayo Clinic, Scottsdale, AZ.

Background: Peripheral nerve sheath tumors (PNSTs) most often occur in the soft tissue or paraspinal region. While PNSTs are relatively common in the posterior mediastinum, they have only rarely been reported in the lung and other thoracic sites, and many of these reports predate the current classification system. Our goal is to more fully characterize the spectrum of PNSTs that occur in the thorax with clinicopathological analysis. Design: Cases were identified by searching the pathology database (surgical and consult cases) for PNSTs occurring in lung, pleura, and mediastinum. Diagnoses were confirmed by slide review and immunohistochemistry as needed. Follow-up was obtained from medical records and referring physicians.
Results: Study cases include 18 benign pleuropulmonary PNSTs, 49 benign mediastinal PNSTs, and 5 malignant PNSTs. The benign pleuropulmonary tumors were composed of 13 schwannomas, 3 neurofibromas, 1 perineurioma and 1 ganglioneuroma. They occurred in 8 men and 10 women (average 54 years, range 25-81). Five of these lesions were endobronchial $(0.3-1.7 \mathrm{~cm}$; 1 schwannoma, 2 neurofibromas, 1 perineurioma, 1 ganglioneuroma), while the remaining schwannomas and 1 neurofibroma formed masses $(1-9.5 \mathrm{~cm})$ that were almost always pleural based. The benign mediastinal PNSTs were all schwannomas (average $5.5 \mathrm{~cm}$, range 1.8-11.6 cm): 46 posterior, 2 middle, and 1 anterior. Mediastinal schwannomas showed a female predominance (34 women, 15 men; average 51 years, range 15-77). Follow-up was available on 48 patients with benign PNSTs (average 43 months, range 1-163), with no recurrence or metastasis. Of the 5 thoracic malignant PNSTs, 4 were pleuropulmonary (typically pleural based) and 1 occurred in the upper anterior mediastinum, with a size range of 5.5-10.4 cm. They occurred in 3 women and 2 men, aged 27-84 years. Two of the 5 patients had a history of neurofibromatosis type 1 (NF-1, aged 27 and 45). Follow-up was available on 2 patients, and both had died of disease ( 5 and 10 months following diagnosis) Conclusions: Although rare, a wide range of PNSTs occur in the lung. While neurofibroma, perineurioma and ganglioneuroma were observed as endobronchial lesions, the vast majority of pleuropulmonary schwannomas were pleural based masses. Mediastinal PNSTs are dominated by posterior mediastinal schwannomas, although schwannomas can rarely occur in the other mediastinal compartments as well. Malignant PNSTs are very rare in the thorax, where they show aggressive behavior. The diagnosis of malignant PNST should prompt evaluation for NF-1, especially in young patients.

1947 Histologic Evolution of Lung Adenocarcinomas with Acquired Resistance to Epidermal Growth Factor Receptor (EGFR) Inhibitors

G Cai, SN Gettinger, Z Walther, AK Wurtz, V Pirazzoli, Y Bao, RJ Homer, K Politi. Yale University School of Medicine, New Haven, CT.

Background: Lung adenocarcinomas harboring epidermal growth factor receptor (EGFR) mutations can be effectively treated with small molecule EGFR tyrosine kinase inhibitors (TKI). However, despite initial responses, most mutated lung cancers develop resistance EGFR TKI, in 40-50\% of such cases as a result of a secondary molecular alteration in EGFR (the T790M mutation). In addition, histologic transformation has been seen in some tumors with acquired resistance to EGFR TKI. Recognition of secondary molecular and histologic alterations may have therapeutic implications. In this study, we evaluated morphologic features in the mutated EGFR lung adenocarcinoma specimens at the time of acquired resistance to EGFR TKI and compared this to the pre-EGFR TKI specimens.

Design: From May 2010 to August 2013, 41 patients with mutated EGFR lung adenocarcinoma and acquired resistance to EGFR TKI were re-biopsied at our institution. Pre- and post-EGFR TKI specimens were available for histomorphologic comparison. In selective cases, immunohistochemical studies were performed to confirm morphologic evaluation.

Results: Of 41 patients, 8 individuals (20\%) showed acquired resistance to EGFR TKI with morphologic evolution, 4 to 21 months (average 12 months) after initiation of EGFR TKI, transforming from well or moderately differentiated adenocarcinoma to either small cell carcinoma (4 cases) or carcinoma with sarcomatoid features (4 cases). Small cell carcinoma transformation was characterized by small to intermediate sized tumor cells with high nuclear to cytoplasmic ratios and speckled chromatin and further supported by immuno-reactivity with chromogranin, synaptophysin and CD56. The carcinomas with sarcomatoid features showed pleomorphic large tumor cells with abundant cytoplasm and frequent multinucleated giant tumor cells, and had focal vimentin expression and aberrant cytoplasmic E-cadherin expression. All of the tumors with morphologic transformation had identical EGFR mutations as identified in the pre-EGFR TKI specimens but were negative for secondary T790M mutation. Conclusions: Morphologic transformation of lung adenocarcinomas can be seen in a subset of patients who develop resistance to EGFR TKI. Histologic evolution includes small cell carcinoma or sarcomatoid transformation, the underlying molecular mechanisms of which are yet to be determined.

1948 Detection of Human Papillomavirus in Non-Small Cell Carcinoma of the Lung: A Study of 199 Cases

SY Chang, M Keeney, J Donovan, J Garcia, M-C Aubry. Sunnybrook Health Sciences Centre, Toronto, Canada; Mayo Clinic, Rochester, MN.

Background: High-risk human papillomavirus (hrHPV) is an etiologic agent in carcinomas arising in sites such as oropharynx, cervix (squamous cell carcinoma; $\mathrm{SqCC}$ ), endocervix (adenocarcinoma; $\mathrm{ADC}$ ), and a proven prognostic factor in the oropharyngeal SqCC. Many studies have detected HPV in NSCLC (ranging widely from 0 to $78 \%$ ) with high rate also seen in ADC occurring mostly in East Asian population (e.g. $49.4 \%$ in a Taiwanese study). Common methods used to detect HPV include: polymerase chain reaction, Hybrid Capture and in-situ hybridization (ISH) for HPV DNA, and immunhistochemistry (IHC) for p16. Recent studies advocate for the detection of mRNA transcripts of E6/E7 as a more reliable evidence of transcriptionally active HPV in tumor cells. The clinical significance of finding HPV remains unclear in NSCLC, especially in light of the various methods used. This study sought to determine the rate of hrHPV infection in NSCLC and its clinical significance at our institution. Design: A total of 199 resected primary lung ADC $(n=100)$ and SqCC $(n=99)$ were retrieved to construct tissue microarray blocks (three $1 \mathrm{~mm}$ cores harvested per case). High-risk HPV DNA ISH and IHC for p16 (INFORM HPV III Family 16 probe and CINtec mouse monoclonal antibody clone E6H4, respectively, Ventana Medical Systems, Tuscan, AZ) were performed. Cases of oropharyngeal SqCC with known hrHPV infection were used as positive controls. P16 expression was scored 
as positive if $\geq 70 \%$ of tumor cells showed diffuse nuclear and cytoplasmic staining Punctate nuclear hybridization signals by DNA ISH in the malignant cells defined a HPV-positive carcinoma.

Results: Of the 199 cases (111 men, 88 women, median age 71 years, range 33-87 years), p16 was positive in 19 ADC and 10 SqCC, but hrHPV DNA ISH was negative in all cases. There is no correlation between positive p16 expression and the following parameters: age, gender, smoking status, tumor size, histologic type, grade and stage. Conclusions: Our study did not detect HPV infection by hrHPV DNA ISH in any of the primary NSCLC despite positive p16 expression in a portion of both ADC and SqCC. P16 should therefore not be used as a surrogate marker for HPV infection in NSCLC.

1949 Frequency of TERT Promoter Mutations in Squamous Cell Carcinomas of Different Origins

$K$ Cheng, J Zhuge, PMR Ravella, $R$ Lafaro, $M$ Zhong. Westchester Medical Center/ New York Medical College, Valhalla, NY.

Background: Telomerase reverse transcriptase (TERT) is up-regulated in a variety of human neoplasms and contributes to carcinogenesis. TERT promoter mutations were recently discovered in melanoma by next generation sequencing. Studies have indicated that, these mutations are highly prevalent in melanoma, glioblastoma, and bladder cancers, and low frequency in non small cell lung cancer (NSCLC) cell lines. However, these findings have not yet been illustrated TERT promoter mutation status in squamous cell carcinoma (SCC) of the lung as well as other primary SCC. Our aim was to investigate whether the aforementioned TERT promoter mutations were present in SCC of different origins.

Design: Total 53 cases were selected from the archives of our pathology department. These include 24 lung SCC, 12 head and neck SCC, 9 cervical SCC and 8 skin SCC. All Hematoxylin and Eosin (H\&E) stained slides were reviewed to confirm the diagnosis and make sure at least $>20 \%$ tumor content. In addition, several cases of non neoplastic tissue from the matched SCC patient were also studied. Genomic DNA was isolated from FFPE tissue and PCR was used to amplify TERT promoter region which included the $\mathrm{C} 228 \mathrm{~T}$ and $\mathrm{C} 250 \mathrm{~T}$ mutations. The TERT promoter mutations are determined by bi-directional Sanger sequencing.

Results: The TERT promoter mutations were absent in all of the lung SCC and cervical SCC. In head and neck SCC, only two out of twelve $(2 / 12 ; 17 \%)$ cases had the C228T mutation. For skin SCC, four out of eight $(4 / 8 ; 50 \%)$ cases had the mutations including 3 cases of $\mathrm{C} 228 \mathrm{~T}$ and 1 case of $\mathrm{C} 250 \mathrm{~T}$. No mutations were detected in the matched non-neoplastic tissue.

Conclusions: This is the first study to compare TERT promoter mutations in SCC of different origins. In our study, the TERT promoter mutations were not present in any of the lung and cervical SCC. The frequency of TERT promoter mutation is $17 \%$ and $50 \%$ in head\& neck SCC and skin SCC, respectively. The findings further illustrate molecular differences among SCC from different origins despite their shared morphologic and immunophenotypic features. The TERT promoter mutations could be of utility as a molecular marker to distinguish SCC with different origins.

\section{PIK3CA/AKT Pathway Alterations in Squamous Cell Carcinoma of} the Lung

AWS Chi, SE Johnstone, CI Wang, E Mark, M Mino-Kenudson. Massachusetts General Hospital, Boston, MA

Background: Lung cancer is the leading cause of cancer-related death in the United States. The identification of recurrent genetic alterations involving EGFR, ALK, and $K R A S$ has revolutionized the clinical management of patients with lung adenocarcinoma. The molecular mechanisms underlying the tumorigenesis of lung squamous cell carcinoma (SqCC), however, are less understood. Alterations of the PIK3CA/AKT pathway have been recently reported in up to $47 \%$ of lung SqCC. Given the ongoing development of protein kinase inhibitors as potential therapeutic agents, we evaluated alterations in the PIK3CA/AKT pathway in lung SqCC.

Design: Tissue microarrays were constructed from 145 surgically resected lung SqCC specimens ( 2 cores of each case; $61 \%$ in stage 1, 26\% in stage 2, $12 \%$ in stage 3 , and $1 \%$ in stage 4 ). The preservation or loss of PTEN expression was determined by immunohistochemistry. 80 cases were further analyzed by a clinically validated multiplexed PCR based assay to detect specific single nucleotide mutations in PIK3CA (8 variants) and $A K T 1$ (1 variant). Correlations between genetic mutations, protein expression, and clinicopathological characteristics were assessed by log-rank and Fisher's exact tests.

Results: PIK3CA mutations were identified in $7(9 \%)$ of the 80 specimens. No cases harbored the tested AKT1 mutation. Loss of PTEN expression was found in $25 \%$ of the cases and one case had concurrent PIK3CA mutation and PTEN loss. In total, 35\% of cases analyzed with both multiplexed PCR and PTEN immunohistochemistry harbored PIK3CA/AKT pathway alterations. These alterations, however, were not associated with specific clinicopathological features, including survival.

Conclusions: A significant fraction (1/3) of lung squamous cell carcinomas harbor alterations in the PIK3CA/AKT pathway Testing of these biomarkers in SqCC may become useful in the future as multiple inhibitors of this pathway are being examined in clinical trials.

1951 Evaluation of BCL2 and BIM Expression and KRAS Mutations in Lung Adenocarcinoma

$A W$ Chi, SE Johnstone, $R$ Heist, E Mark, M Mino-Kenudson. Massachusetts General Hospital, Boston, MA.

Background: Although activating KRAS mutations are the most common driver mutations in lung adenocarcinoma of the western population, how KRAS mutations drive tumorigenesis is not completely understood. Several preclinical studies have shown that KRAS mutations suppress a pro-apoptotic protein, BCL2 interacting mediator of cell death (BIM), and subsequently facilitate proliferation. However, little is known about the expression of BIM and other pro-apoptotic and anti-apoptotic proteins in association with clinicopathological characteristics in lung adenocarcinoma. Here, we analyzed the expression of BIM and BCL2, an anti-apoptotic factor, in our molecularly annotated lung adenocarcinoma cohort.

Design: Eight tissue microarrays are constructed from 158 surgically resected lung adenocarcinoma specimens ( $3 \%$ stage $0,72 \%$ stage $1,16 \%$ stage 2 , and $9 \%$ stage 3 ). All specimens were submitted for multiplex PCR analysis to detect specific single nucleotide mutations in multiple genes, including $K R A S$. The expression of BIM and BCL2 was analyzed using H-score by immunohistochemistry. Appropriate cut-off for positive expression was determined. Correlation between protein expression and genetic mutations, clinicopathological characteristics and survival was assessed by Fisher's exact and log-rank tests.

Results: Positive expression of BIM was seen in 53\% and 59\% of KRAS mutants, $33 \%$ and $29 \%$ of $E G F R$ mutants and $46 \%$ and $53 \%$ of tumors without $K R A S$ or $E G F R$ mutations, in the entire and stage 1 cohorts, respectively. BIM expression was more prevalent in the KRAS mutants, especially in the early stage tumors, compared to the $E G F R$ mutants $(p=0.017)$. The expression of BCL2 was limited in our cohort $(19 \%)$ and there was no correlation between BCL2 expression and molecular alterations. Interestingly, there was a positive association between BCL2 and BIM expression in epithelial cells of lung adenocarcinomas $(p=0.036)$. However, there was no significant difference in the clinicopathological characteristics or survival between BIM+ vs. BIM-, BCL2+ vs. BCL2-, or BIM+BCL2- vs. BIM-BCL2+ tumors.

Conclusions: Our study shows counterintuitive correlation between BIM expression and KRAS mutations in resected lung adenocarcinomas, highlighting the complex interactions between pro- and anti-apoptotic molecules. It further suggests possible involvement of other pathways in the KRAS-BIM axis. The absence of correlation between BIM and BCL2 expression with prognosis warrants the need for further investigation of these and other pro- and anti-apoptotic proteins in tumor biology.

1952 The Prevalence and Implication of Adenocarcinoma Component in Lung Cancer with Morphological Diagnosis of Squamous Cell Carcinoma A Chi, S Johnstone, M Nishino, E Mark, M Mino-Kenudson. Massachusetts General Hospital, Boston, MA; Beth Israel Deaconess Medical Center, Boston, MA.

Background: Molecular alterations commonly associated with lung adenocarcinoma (ACA) are also seen in adenosquamous carcinoma ( $\mathrm{ASqC}$ ). Currently, the diagnosis of lung $\mathrm{ASqC}$ requires the presence of both squamous cell carcinoma $(\mathrm{SqCC})$ and $\mathrm{ACA}$ components on histologic examination with each comprising at least $10 \%$ of the tumor. However, the significance of a minor ACA component $(<10 \%)$ in otherwise classic $\mathrm{SqCC}$ in association with molecular alterations is not well understood. In addition, the prevalence of lung cancers that are morphologically diagnosed as SqCC but harbor an ACA immunophenotype has not been well documented.

Design: A prospective database of resected lung cancers was queried to identify patients with $\mathrm{SqCC}$ who underwent primary resection without neoadjuvant therapy since 2005 . Of those, cases with the pathology diagnosis made solely by morphology (without ancillary tests), and available tissue blocks ( $\mathrm{n}=154)$ formed our study cohort. Each case was evaluated for the presence of any glandular differentiation. Mucicarmine stain was used to confirm the presence of mucin in some cases. Analysis of TTF-1, Napsin A, CK5/6, and p40 expression was performed on tissue microarrays. As part of clinical care at our institution, SNaPshot (a multiplex PCR-based platform for common driver mutations) was also performed in 84 cases.

Results: The 4-marker panel immunohistochemical analysis supports the diagnosis of ACA (with a solid pattern) in 10 tumors $(6.5 \%)$. Of these, the retrospective review revealed the presence of mucin in 3 . The review also identified 12 additional cases $(7.8 \%)$ with a minor ACA component $(<10 \%)$. SNaPshot assay was performed in 14 of the 22 cases, and showed KRAS mutations in one of solid ACAs $(1 / 5,20 \%)$ and one of SqCCs with a minor ACA component $(1 / 9,11 \%)$. Interestingly, another case of the latter group harbored an $\mathrm{IDHI}$ mutation.

Conclusions: Misinterpretation of ACA with a solid component as $\mathrm{SqCC}$ and the presence of a minor ACA component in otherwise classic SqCC (possible ASqC) are not infrequently encountered in lung cancers with morphological diagnosis of SqCC. Given the clinical importance of the distinction, greater emphasis should be placed during morphological evaluation and immunohistochemistry should be applied in any questionable cases to improve the sensitivity for detecting an ACA component.

1953 Comparison of Direct Sequencing and Peptide Nucleic Acid Clamping of EGFR Gene in Patients with Non-Small Cell Lung Cancer Y-D Choi, C-S Park, J-H Nam, S-S Kim, Y Kim, J-H Kim. Chonnam National University Medical School, Gwangju, Republic of Korea.

Background: Direct sequencing is considered as a gold standard for the detection of epidermal growth factor receptor (EGFR) gene mutation in non-small cell lung cancer (NSCLC), but low sensitivity is a problem. The aim of this study is to prove higher detection rate of EGFR mutation with peptide nucleic acid (PNA) clamping compared with sequencing.

Design: This is single arm, open label, prospective, observational study for patients with stage IIIB or IV NSCLC. From January 2013, tumor DNA samples from 117 patients were obtained from paraffin block or cytology specimen. Both sequencing and PNA clamping for EGFR gene in exon 18, 19, 20 and 21 were performed.

Results: Of 117 paired test sets, 21 mutations of EGFR gene were detected by sequencing $(17.9 \%)$ and 41 mutations were detected by PNA clamping $(35.0 \%$, $\mathrm{p}<0.001)$. The concordance rate between two methods was $78.1 \%(\kappa=0.552, \mathrm{p}<0.001)$. Of 37 patients with EGFR mutation by PNA clamping, there were 17 partial responses (PR), 3 stable diseases (SD), 2 progressive diseases (PD), and 15 cases were not 
evaluable (NE) after treatment with EGFR-tyrosine kinase inhibitor (TKI). And there were 7 PR, 2 SD, 1 PD and $10 \mathrm{NE}$ among 20 mutation positive patients by direct sequencing method. The response prediction rate to EGFR TKI was $77.3 \%$ by PNA clamping and $70.0 \%$ by sequencing $(\mathrm{p}=0.583)$.

Conclusions: These results showed higher detection rate of EGFR mutation by PNA clamping compared with direct sequencing and moderate concordance between two methods. PNA clamping could be more useful screening method for detection of EGFR mutations

1954 Non-Small Cell Lung Carcinoma Brain Metastases with Squamous Cell Carcinoma or Small Cell Carcinoma Histology

ML Cowan, WH Westra, PB Illei. Johns Hopkins Medical Institutions, Baltimore, MD. Background: Tissue diagnosis of stage IV lung carcinoma is frequently made on biopsies of metastases including solitary brain metastases that are often excised for both diagnostic and therapeutic purposes. The majority of brain metastases have adenocarcinoma (ACA) histology, while a minority can have adenosquamous carcinoma (ADSQ), squamous cell carcinoma (SQC) or small cell carcinoma (SCC) histology, Not infrequently the metastatic tumor remains the only tumor sample available for histologic/molecular analysis. For these minority brain metastases that show ADSQ, SQC or SCC histology, it is not known whether this morphology reliably reflects the histologic subtype of the primary lung cancer.

Design: The archives of our department were searched for patients with brain metastases of SCC, SQC and ADSQ of the lung (1980 to August 2013) who also had their primary tumors sampled. We then compared the reported histologic types of the primary and metastatic tumors and analyzed the lung cancer mutation results if available.

Results: Of 106 patients with metastatic small cell carcinoma in the brain, 11(10.4\%) had tissue sampling of the lung primary. Of these, 3 ( 3 of $11-34 \%$ ) showed different morphology in the lung (1 squamous cell carcinoma, 1 adenocarcinoma, and 1 combined adenocarcinoma-small cell carcinoma). Mutation analysis of the latter tumor demonstrated the same KRAS mutation (G12A) in both ACA and SCC component of the lung primary and in the brain metastasis. Of 89 patients with metastatic squamous cell carcinoma in the brain, $9(11.2 \%)$ had lung tissue sampling, and all 9 were SQC $(100 \%)$. Interestingly, we also identified 5 metastatic ADSQ; 3 with ADSQ primary, 1 with large cell undifferentiated carcinoma primary and 1 with an EBUS mediastinal lymph node biopsy showing SQC. One brain metastasis showed mixed SQC-SCC histology and had a primary mediastinal SCC primary. One of the metastatic ADSQ had an exon 19 (E746 A750del5) EGFR while the mutation status of the others is not known. Conclusions: These findings suggest that at least a small subset of patients with non-small cell lung carcinoma present with SCC brain metastasis. The relatively low frequency of these cases raise the question whether mutation studies should be performed on all brain metastasis of suspected lung tumors where the primary tumor is not or sub optimally sampled in order to ensure appropriate therapy. Mutation studies of all these tumors could provide important data to better understand this process.

1955 PD-L1 Expression in Malignant Mesothelioma: An Immunohistochemical Analysis of $\mathbf{3 3}$ Cases

ML Cowan, PM Forde, JM Taube, J Brahmer, PB Illei. Johns Hopkins Hospital, Baltimore, MD.

Background: Malignant mesothelioma is an aggressive neoplasm with 43,000 deaths worldwide and no effective systemic therapy. Since most mesotheliomas are associated with asbestos exposure and chronic inflammation they are potentially "immunogenic tumors". Novel immune checkpoint inhibition of programmed death - 1 (PD1) targeted therapy has shown promise in other solid tumors including non-small-cell lung cancer (NSCLC) and malignant melanoma. Mesothelioma mouse model data suggest high expression of programmed death- ligand 1 (PD-L1), the lead candidate biomarker of anti-PD-1 therapy but there is no data available regarding expression levels in primary human mesotheliomas. Here we report the rate of PD-L1 expression in malignant mesothelioma and in associated tumor infiltrating lymphocytes.

Design: Immunohistochemistry for PD-L1 (murine anti-human PDL1 monoclonal antibody 5H1) and $\mathrm{CD} 3$ (mouse monoclonal antibody, clone PS1) was performed on standard formalin fixed paraffin embedded tissue sections of large biopsy or resection specimens of 25 of pleural (17 epithelioid, 7 biphasic \& 1 sarcomatoid; median age: 65 ) and 8 peritoneal (all epithelioid, 2 in tunica vaginalis, median age: 72.5 ) mesotheliomas. Based on earlier published studies tumor cells or tumor infiltrating lymphocytes were considered positive if at least 5\% exhibited membranous staining. The CD3+ tumor infiltrating lymphocytes (TIL) were estimated as percent of cells within the tumor and the presence and location of lymphoid aggregates as well as percent of PD-L1 positive TIL was also evaluated.

Results: The tumor cells were PD-L1 negative in 10 cases while positive in 23 cases ( 1 had $5 \%$ staining, 8 had $6-49 \%$ staining and $14 \mathrm{had} \geq 50 \%$ staining). The majority of tumors exhibited geographic (primarily peripheral zonal) staining but diffuse staining was also noted. PD-L1 positivity was noted in $50 \%$ of peritoneal and $76 \%$ of pleural mesotheliomas. The majority ( $83 \%$ ) of PD-L1 positive tumors also had PD-L1 positive TIL (range of PD-L1 positive TIL: 5-80\%) and lymphoid aggregates (74\%). TIL were seen less often (40\%) in PD-L1 negative tumors (range of PD-L1 positive TIL: 10-60\%). Conclusions: The majority of malignant mesotheliomas are PD-L1 positive by immunohistochemistry and contain PD-L1 positive TIL. Pleural mesotheliomas are more often positive than peritoneal mesotheliomas ( $76 \%$ vs. $50 \%$ ). These findings suggest that malignant mesothelioma is potential candidate for anti-PD-1 therapy.
1956 Evaluation of Small Cell Undifferentiated Lung Cancer by Next Generation Sequencing Reveals Frequent Consistent Genomic Alterations OR Elkadi, A Tarasen, LX Foulke, CE Sheehan, K Wang, GA Otto, G Palmer, R Yelensky, D Lipson, J Chmielecki, SM Ali, D Morosini, VA Miller, PJ Stephens, JS Ross. Albany Medical College, Albany, NY; Foundation Medicine Inc, Cambridge, MA.

Background: Although the response rate to standard chemotherapy for small cell undifferentiated lung carcinoma (SCLC) is high, the clinical outcome is poor with a 5 year overall survival rate of only $5 \%$. As opposed to other types of primary lung cancer, most notably lung adenocarcinoma, well defined genomic alterations and opportunities for targeted therapy for SCLC have not, to date been identified. We hypothesized that comprehensive genomic profiling of clinical SCLC samples by NGS could identify genomic-derived drug targets of therapy for patients diagnosed with this aggressive malignancy in a single diagnostic test.

Design: Hybridization capture of 3,769 exons from 236 cancer-related genes and 47 introns of 19 genes commonly rearranged in cancer (current version of the test) was applied to $\geq 50 \mathrm{ng}$ of DNA extracted from 49 SCLC FFPE tumor specimens and sequenced to high, uniform coverage. Genomic alterations (base substitutions, small indels, rearrangements, copy number alterations) were determined and then reported for these patient samples. Actionable GA were defined as those identifying anti-cancer drugs on the market or in registered clinical trials (CT).

Results: There were 31 female and 18 male SCLC patients with a median age 57.5 years (range 32-83 years). All tumors were high grade, and 21 tumors were stage IV, 24 stage III, 2 stage II and 2 stage 1 at time of sequencing. All $49(100 \%)$ of SCLC had GA on NGS with a total of 149 GA were identified for an average of 3.04 GA per tumor with $100 \%$ of SCLC cases harboring at least one alteration. The most common non-actionable genomic alterations were alterations in TP53 $(71 \%), R B 1 \mathrm{i}(67 \%)$ and MLL2 (16\%). Twenty one (43\%) of cases harbored at least 1 actionable GA including mutation, amplification or homozygous deletion in EGFR (6\%), PIK3CA (6\%), PTEN (6\%), RICTOR (6\%), TSCl (6\%) and NOTCHI (4\%), BRAF (2\%), BRCA2 ( $2 \%)$, CCNDI (2\%), CCND3 (2\%), CCNE1 (2\%), CDKN2A (2\%), CDK4 (2\%), FGFR1 (2\%), KRAS $(2 \%), N F 1(2 \%)$ and $T S C 2(2 \%)$

Conclusions: More than third of the patients harbored at least one actionable GA. The long tail of altered genes and multiple mechanisms of alteration necessitate broad diagnostic assays from limited biopsy material to maximize targeted therapeutic options in an individual patient. Given the limited treatment options and poor prognosis of patients with SCLC, comprehensive NGS-based genomic profiling has the potential to identify new treatment paradigms and meet an unmet clinical need for this disease.

1957 SOX2 Expression in EGFR and KRAS Mutated Lung Adenocarcinomas

NFatima, K Fisher, MR Rossi, L Zhang, T Owanikoko, SRamalingam, F Khuri, A Gal, $C$ Cohen, G Sica. Emory University Hospital, Atlanta, GA.

Background: SOX2, is a transcription factor associated with maintaining the pluripotency and self renewal capacity of embryonic stem cells. It is preferentially amplified in lung adenocarcinomas from never smokers, may be an adverse prognostic marker. SOX2 is also a key downstream target of EGFR signal, but can be down regulated in a murine model of KRAS induced lung adenocarcinoma. Herein we investigated SOX2 expression in a set of lung adenocarcinomas with and without mutations in a set of key oncogenes.

Design: Based on previous reports, nuclear staining for SOX2 (goat polyclonal, R\&D systems) was scored as positive or negative using a 5\% cutoff. Tumors were assessed for point mutations by multiplexed PCR, single primer extension, and capillary electrophoresis. PCR amplification and capillary electrophoresis based size assessment were used to determine the presence of insertions and deletions in EGFR and HER2. Results: Of the 29 cases analyzed, $87.5 \%(14 ; n=16)$ of the cases with mutations and $92.3 \%(12 ; n=13)$ without mutations were SOX2 positive. Of the SOX2 positive cases with mutations, $100 \%$ of the EGFR (6), BRAF (2) and Her2 (1) mutated cases were SOX2 positive while $71.4 \%(5, n=7)$ of the KRAS cases were positive.

Conclusions: SOX2 is expressed in lung adenocarcinomas with EGFR, BRAF and Her2 mutations, but may not be expressed in KRAS mutated adenocarcinomas, raising the possibility that not all KRAS mutated tumors utilize SOX2 mediated cancer cell renewal.

1958 Molecular Detection of Clinically Actionable Lung Cancer Mutations: Multiplexed Single Gene Assays Versus Next-Generation Sequencing Following Target-Enrichment

KE Fisher, J Wang, RN Pillai, TK Owonikoko, SR Ramalingam, FR Khuri, AA Gal, KP Mann, CE Hill, GL Sica, MR Rossi, L Zhang. Emory University, Atlanta, GA.

Background: Accurate characterization of clinically actionable mutations in lung carcinomas is essential for optimal targeted molecular therapy and patient enrollment into novel clinical trials. For non-small cell lung cancers (NSCLC) at our institution, we test for $E G F R$ and $H E R 2$ insertions and deletions (EGFR/HER2 Indels) and select point mutations in AKT1, BRAF, EGFR, IDH1, IDH2, KRAS, MEK1, NRAS, PIK3CA, and PTEN (SNaPshot). We compared the performance of SNaPshot plus EGFR/ HER2 Indels to the Illumina ${ }^{\circledR}$ TruSight ${ }^{\mathrm{TM}}$ Tumor Panel, a target enrichment kit for the MiSeq instrument that covers 26 genes (175 amplicons) consistent with CAP and NCCN guidelines.

Design: We tested 19 formalin-fixed paraffin embedded (FFPE) NSCLC specimens. DNA was extracted with a Qiagen FFPE extraction kit after tumor quantification and macrodissection. SNaPshot was performed using multiplexed PCR, single primer extension, and capillary electrophoresis (CE). EGFR/HER2 Indels were determined by PCR amplification and size assessment by $C E$. Tumor DNA next-generation sequencing (NGS) amplifiability (QC) was assessed by quantitative PCR. NGS libraries were 
prepared with the Illumina ${ }^{\circledR}$ TruSight ${ }^{\mathrm{TM}}$ Tumor kit and run on a MiSeq. Sequence alignment, variant calls and data interpretation were performed using on-board MiSeq software, Illumina ${ }^{\circledR}$ VariantStudio ${ }^{\mathrm{TM}}$ and IGV 2.3 .

Results: SNaPshot and $E G F R / H E R 2$ Indels testing were performed on all 19 cases. We detected 7 cases with point mutations ( $5 K R A S, 1 E G F R$, and $1 B R A F)$ and two cases with HER2 insertions. Sixteen cases met NGS QC and were prepped with the TruSight Tumor kit. NGS of the TruSight libraries confirmed all 7 SNaPshot point mutations and detected additional mutations affecting TP53 and STK11 genes in two cases that SNaPshot detected a single KRAS point mutation. Furthermore, NGS of the TruSight libraries identified mutations in 7 cases that were negative by SNaPshot and $E G F R /$ HER2 Indels: TP53 alone (3), KRAS alone (1), KRAS plus TP53 (3), and CTNNB1 plus $A K T 1$ (1 fetal adenocarcinoma case). NGS QC failed for both HER2 insertion cases. Two cases had no mutations by either method.

Conclusions: The TruSight ${ }^{\mathrm{TM}}$ Tumor kit identified potentially clinically actionable mutations that were not detected by SNaPshot in almost half of the tested specimens. The TruSight ${ }^{\mathrm{TM}}$ Tumor kit offers an attractive alternative to our current testing methodologies, especially for cases that meet the stringent DNA QC standards required for NGS.

1959 Lung Adenocarcinoma Subtype (Terminal Respiratory Unit (TRU) vs. Non-TRU ) and Association with KRAS, EGFR, BRAF and HER2 Gene Mutations

KE Fisher, N Fatima, RR Micahael, Z Linsheng, K Sungjin, C Zhengjia, OK Taofeek, RS Suresh, K Fadlo, GA Anthony, SL Gabriel. Emory University School of Medicine, Atlanta, GA.

Background: TRU lung adenocarcinomas that recapitulate the lung alveolar and non ciliated bronchiolar epithelium are associated with activating mutations in the tyrosine kinase domain of the EGFR gene. However, not all TRU adenocarcinomas harbor mutations in the EGFR gene and the genomic profile of non-TRU adenocarcinomas have not been well defined. We investigated differences in the genomic profile of TRU and non-TRU lung adenocarcinomas and assessed for associations between these two morphologic subtypes and genomic characteristics.

Design: We classified lung adenocarcinomas using the previously published morphologic criteria for TRU and non-TRU. Cases that were not classifiable into either group were coded as mixed. Point mutations were assessed by multiplexed PCR, single primer extension, and capillary electrophoresis. Insertions and deletions in EGFR and HER 2 were determined by PCR amplification and size assessment after capillary electrophoresis. Univariate association between the mutation status and TRU and non TRU histology was examined with Fisher's exact test using SAS 9.3 (SAS Institute, Inc., Cary, North Carolina) with a significant level of 0.05 .

Results: There were 41 cases of lung adenocarcinoma and 1 case of adenosquamous carcinoma included in this analysis. Sixteen cases were classified as TRU, 22 as nonTRU and 4 as mixed. EGFR mutation was detected in 6 of 42 informative cases (14.2\%) while KRAS mutation was present in 7 of 29 informative cases $(24.1 \%) .10 .3 \%(n=3)$ of 29 cases had activating mutations in other oncogenes, including a deletion in Her2 and point mutations in BRAF. KRAS mutation was more frequent in non-TRU vs TRU adenocarcinomas $(42.9 \%$ vs. $7.7 \%$; $\mathrm{p}=0.077)$ while non KRAS mutations, including EGFR, were more likely to occur in TRU adenocarcinomas $(53.9 \%$ vs. $7.1 \% ; \mathrm{p}=0.013)$. Conclusions: Non-TRU adenocarcinomas are more often associated with KRAS mutation, while TRU adenocarcinomas are more often associated with non KRAS mutations, including EGFR, Her2 and BRAF. The correlation of morphologic subtypes of lung adenocarcinoma with distinct driver genomic alterations suggests a fundamental difference in the morphologic and genomic evolution of these histologically divergent tumor subtypes.

1960 Organizing Pneumonia and Smooth Muscle Proliferation Is a Frequent Histopathologic Finding in Grossly Normal Areas of Lungs Explanted for Usual Interstitial Pneumonia

DJ Fix, J Collins, AP Burke. University of Maryland Medical Center, Baltimore, MD. Background: The histologic findings of uninvolved lung parenchyma in patients with UIP requiring lung trasnplantation has not been well studied. Our goal is further describe these findings based on histologic patterns and immunohistochemical staining. Design: We prospectively studied 19 single lung transplants for UIP, and sampled 4 (in most cases) grossly normal areas from all lobes. There were 18 men and 1 women, 14 right and 5 left lung transplants. Sagittal sections were performed after inflating with formalin fixative, and gross photographs taken in each case. Histologic features of $\mathrm{OP}$ were described by location as interstitial, paraseptal, or peribronchiolar. Immunohistochemical staining for anti-smooth muscle actin antibody (SMA) and antidesmin antibody (DES) was performed on all sections, and patterns of staining regarded as 'above normal levels', as well as categorized by basic histologic patterns defined as: Interstitial and Nonspecific interstitial pneumonitis-like (NSIP-like) (positive for SMA, with rare focal areas of desmin positivity), areas of OP (positive for SMA with variable DES staining), and nodular proliferations with smooth muscle hyperplasia (positive for both SMA and DES).

Results: Of the 73 sections examined, OP-like areas were present in 57 (78\%), with all lungs containing at least one focus. There were 230 foci of OP in total, with 136 interstitial (59\%), 73 paraseptal $(32 \%)$, and 21 peribronchiolar $(9 \%)$. There were numerous foci of chronic interstitial pneumonitis with areas of inflammation and fibrosis and abundance of SMA positive cells suggestive of a NSIP-like pattern, identified in $17 / 19(89 \%)$ of cases comprising $5-100 \%$ of tissue present. Immunohistochemical staining patterns were observed as follows: Interstitial/NSIP-like in 15/19 (79\%), OP-like areas in $11 / 19(58 \%)$, nodular proliferations with smooth muscle hyperplasia in $6 / 19(32 \%)$ of cases.
Conclusions: Normal areas in UIP lungs frequently exhibited areas of OP, NSIP-like changes, as well as nodular proliferations of smooth muscle hyperplasia in a lesser but significant proportion. Underlying these changes are increases in SMA and DES in reproducible patterns.

\section{Identification of Novel Somatic Variants in Thymic Epithelial} Tumors by Next-Generation Sequencing

J Fujimoto, J Zhang, CR Pickering, H Kadara, C-G Liu, JA Drummond, DM Muzny, $H$ Doddapaneni, A Weissferdt, DA Wheeler, JN Myers, J Wang, II Wistuba, N Kalhor, CA Moran. The University of Texas MD Anderson Cancer Center, Houston, TX; Baylor College of Medicine, Houston, TX

Background: Thymic epithelial malignancies mainly comprise thymomas and thymic carcinomas and represent about $1 \%$ of all epithelial tumors. Our knowledge on the molecular makeup and pathogenesis of thymic tumors is substantially lacking. In this study, we sought to characterize exonic mutations in a limited set of thymic tumors by next-generation sequencing (NGS)

Design: We performed whole-exome sequencing of 5 frozen paired thymic tumors ( 3 thymomas and 2 thymic carcinomas) and adjacent normal tissues using the Illumina HiSeq 2000 platform. Exon capture was performed using the Nimblegen exon capture array. Variants that were detected in normal tissue, silent and reported in dbGAP and 1000 genomes were filtered out to identify somatic exonic non-synonymous alterations. Top calls were then selected based on involvement in known cancer-related pathways. Identified top somatic variants were analyzed and confirmed in 22 formalin-fixed paraffin embedded (FFPE) epithelial rich thymic tumors 14 thymomas and 8 thymic carcinomas) by Ion Torrent sequencing using the PGM platform on Ion 318 chips and the AmpliSeq comprehensive cancer panel. Select variants were then visualized by the Integrative Genomics Viewer (IGV) software.

Results: We identified, using whole-exome sequencing, candidate somatic alterations in 59 genes comprised of 1 frame shift insertion, 8 deletions as well as 50 single nucleotide variants (SNVs). The highest number of somatic alterations per tumor was 27 (in a thymic carcinoma). Top variants included a missense mutations in harvey rat sarcoma viral oncogene homolog (HRAS), carbonic anhydrase 6 (CA6), guanine nucleotide binding protein alpha 11 (GNAII), jumonji AT rich interactive domain 2 (JARID2) and a deletion in the multiple endocrine neoplasia 1 (MEN1). Additionally, we identified using a more conserved base calling approach SNVs in ataxia telangiectasia mutated $1(A T M)$ and neurofibromin $1(N F 1)$ tumor suppressors. Notably, Ion Torrent sequencing confirmed exonic somatic mutations in the HRAS oncogene ( 2 thymomas) and $A T M$ tumor suppressor ( 1 thymoma) and identified an additional somatic alteration in NRAS in a thymoma.

Conclusions: Our whole-exome and targeted NGS analyses identified previously uncharacterized somatic alterations in a limited set of thymic tumors and point to potential activation of RAS oncogenes in the pathogenesis of this rare epithelial malignancy. Current efforts are underway to analyze a larger set of thymic tumors by NGS

\section{FGFR1 Gene Amplification in Squamous Cell Bronchopulmonary} Carcinoma

F Galateau Salle, N Le Stang, M Grimaux, VLeon, K Kampbell, Y Oulkhouir, S Lecot Cotigny, E Bergot, G Zalcman. UMR1086 INSERM, Caen, Noormandy, France; UMR1086, Caen, Noormandy, France.

Background: Fibroblast growth factor receptor 1 (FGFR1) gene amplification has been recently reported in 10 to $20 \%$ of primary squamous lung cancer(SCC). To date, it is the main actionable molecular target occurring in lung SCC. Whether FGFR1 amplification could help to select patients for specific FGRF tyrosine kinase inhibitors remains unknown. The aims of this study is to assess the actual frequency of FGFR1 amplification in a series of SCC consecutive patients, from a University Hospital with clinical, demographic characteristics and smoking exposure correlations.

Design: 82 SCC were identified from the charts of the thoracic oncology Department of Caen University Hospital. For all patients medical records, demographic characteristics, smoking history, treatment details were collected from march 2005 to February 2013. All cases were tested for FGFR1 FISH analysis using the FISH Zytolight SPE FGFR1/ CEN8 DUAL color probe on paraffin embedded tumor tissue. Amplification was positive when the ratio was $>2.2$. The variables were compared with bilateral exact Fisher and with Mann- Whitney tests.

Results: Among 82 SCC patients, $85 \%$ were male, $15 \%$ female, median age 63 years. Current smokers were $45 \%$, former smoker $54 \%$, never-smoker $1 \%$, with an average of 40 Pack/years. WHO PS $0 / 1 / 2$ patients were respectively $31 \%$, $45 \%$, and $24 \%$. Stage IV patients were $63 \%$. FGFR1 FISH amplication was observed in $13.5 \%$ of cases. There was no significant statistical difference between sex, age, smoking history and metastatic versus limited stage. While patients with SCC are current or former smokers there was a slight trend of FGFR1 amplification in light smokers/ex smokers. In stage IV patients, PFS did not significantly differ according to FGFR1 status possibly reflecting a lack of power.

Conclusions: Our study shows $13.5 \%$ of FGFR1 amplification in primary SCC lung cancer and should be systematically tested for their putative predictive value when FGFR TKIs will be available. PFS/OS data will be up-dated and presented at the meeting. 
1963 ALK and MET Are Synergistic Co-Activators of Downstream Signals by Amplification in Pulmonary Sarcomatoid Carcinoma: An Achilles Heel for Targeted Therapy?

P Gasparini, G Sozzi, V Ciravolo, S Pupa, E Tamborini, R Caserini, U Pastorino, G Pelosi. Fondazione IRCCS “Istituto Nazionale dei Tumori”, Milano, Italy.

Background: Genetic alterations suitable for targeted therapy are poorly known issues in pulmonary sarcomatoid carcinoma (PSC), an uncommon and life-threatening family of non-small cell lung cancer (NSCLC).

Design: Eighty-six cases of PSC, all formalin-fixed and paraffin-embedded, were assessed for $A L K$ and $M E T$ gene status by FISH and relevant protein expression by immunohistochemistry (IHC) also taking advantage of phosphorylated antibodies for MET. Moreover, $A L K$ and $M E T$ mRNA evaluation by real-time PCR (qPCR) and biochemistry assay by Western Blotting (WB) for downstream activation pathways involving p-MAPK, p-AKT, p-SRC and p-FAK were also carried out.

Results: $A L K$ amplification was found in 15/86 (17\%) and $M E T$ amplification in 21/86 (24\%) PSC, with ALK-amplified tumors also showing MET amplification. A few PSC, however, showed $M E T$ amplification without any $A L K$ gene alteration. Fifty metastatic lung adenocarcinomas used as controls showed ALK or MET amplification in 1 and 1 cases, respectively $(\mathrm{p}=0.015)$. ALK protein expression was always lacking in PSC, while $\mathrm{p}-M E T$ was limited to the relevant amplified tumors. Increased levels of ALK and MET mRNA were instead detected in tumors, especially amplified cases (paired normal lung tissue was always negative). WB assays showed complete activation of downstream signal pathways up to p-SRC and p-FAK recruitment in $A L K / M E T$ co-amplified tumors, whereas isolated $M E T$ amplification or coexisting increased gene copy gain of $A L K /$ $M E T$ was limited to activate $\mathrm{p}-\mathrm{MET}$, $\mathrm{p}-\mathrm{MAPK}$ and $\mathrm{p}-\mathrm{AKT}$.

Conclusions: $A L K$ and $M E T$ genes acted as synergistic, non-random co-activators of downstream signal when co-amplified in a subset of PSC patients, so indicating an oncogene addiction mechanism. These alterations could have a potential for targeted therapy by specific inhibitors.

1964 Type B Thymomas - Relevance of Current Subclassification

$Y$ Gokmen-Polar, W Lu, K Kesler, PJ Loehrer, S Badve. Indiana University School of Medicine, Indianapolis, IN.

Background: Thymomas are rare tumors arising from the epithelial cells of the thymus gland. The current WHO classification categorizes these tumors in to 2 main types, type $\mathrm{A}$ (or Spindle) and type B (or polygonal) based on the nature of the epithelial cells. The latter is further subcategorized into B1, B2, and B3 based on the cellularity and atypia of the epithelial component. The goal of the current study was to analyze the importance of the subclassification of type B thymomas in relation to behavior of the tumors.

Design: Eighty-seven cases of type B thymomas that were seen in our tertiary referral center (in-house and referral cases) for thymic carcinomas and were reexamined by a single pathologist. Clinical data was obtained from our database and correlations were performed with stage of presentation, likelihood of residual disease, distant metastasesfree survival, and likelihood of being free of disease at the end of the followup period. Chi-square test was performed to analyze the statistical significance.

Results: Histological classification showed that the series consisted of 18 B1, 31 B2, and 38 B 3 thymomas. The followup period ranged from 1 month to 21 years (median, 5.2 years). There were no significant differences in the age at diagnosis, presence of autoimmune disease, stage at presentation (stage I/II vs stage III/IV), or likelihood of residual disease after surgery. There were also no significant differences in the likelihood of development of metastases (at 3 or 5 yrs) or being alive without disease for B1, B2, and $\mathrm{B} 3$ tumors. The survival results were similar when the analysis was performed using 2 categories (B1+B2 vs B3 or B1 vs B2+B3) instead of 3 categories.

Conclusions: The data suggest that there are no significant differences in the biological characteristics of subtypes of thymoma type B. In view of the unexpected results, we are expanding the study to all the cases of type B thymomas ( 350$)$ at our institution. Provided the results are confirmed, they challenge the current paradigm and will necessitate a major reconsideration of the classification of these tumors.

\section{BAP1 Sequencing and Immunohistochemistry in a Series of 159} Mesothelioma

M Grimaux, N Le Stang, CNR Mesopath, D Luce, P Hainaut, F Galateau-Salle. CHU CAEN, Caen, Noormandy, France; Institut de Veille Sanitaire, Saint Maurice, Ile de France, France.

Background: Malignant Mesothelioma (MM) is a tumor with dismal prognosis, often associated with exposure to asbestos. MM cases have also been reported in familial clusters and in patients previously treated for cancers such as breast cancer, renal cell carcinoma and melanoma. BAP1 a tumor suppressor gene located on chromosome 3.p21 shows mutations/insertions or $\mathrm{LOH}$ in numerous cancers. We have analyzed the correlation between BAP-1 genetic alterations and immunohistochemistry (IHC) in a series of pleural MM (PMMs) associated with and without history of other cancers. Design: A total of 159 strictly ascertained PPMs were selected from the MESOPATH (French mesothelioma study panel). This series included 83 PMM cases with previous history of cancer (PMM-cancer-group: 67 breast cancer, 7 renal cell carcinoma and 8 melanoma 1 with breast and melanoma) and 76 PMM cases without previous history of cancer (PMM-no cancer group). BAP-1 nuclear staining (BAP1-NS) was detected using anti BAP1 (clone C-4, from Santa Cruz). BAP-1 was scored as positive or negative. Genetic alterations were analyzed in a subset of 33 PMM cases (24 MMS with cancer and 9 without previous cancer). DNA from PMMs was amplified by PC (exon 1 to exon 17) and amplified products were analyzed by direct sequencing. Survival was evaluated with Kaplan Meier analysis and log rank tests.

Results: A total of 110/ 159 cases were negative for BAP-NS with a tendency for a lower proportion of negative in the PMM-cancer versus PMM-no cancer group ( $60 \%$ vs
$79 \%, \mathrm{p}=0.001$ ). BAP1 gene alterations were detected in 10/20 BAP1-NS negative cases, and in 0/10 BAP1-NS positive cases, no difference between PMM-cancer and PMMno cancer groups. Gene alterations included 7 mutations and 3 deletions/insertions. Negative BAP1-NS was associated with a trend to a worse prognosis (median overall survival 14 months in BAP1-NS negative versus 19 months in BAP1-NS positive cases). Conclusions: Loss of BAP-1-NS might predict worse prognosis of PMM. About $50 \%$ of BAP1-NS negative harbor BAP-1 gene alteration. Thus, BAP-1 IHC is not predictive of mutation status, although no false positive (i.e BAP-1-NS positive with BAP-1 mutation) is observed. Patients with previous history of cancer tend to be more frequently negative for BAP-1-NS.

1966 Clinicopathologic Features of Granulomatous-Lymphocytic Interstitial Lung Disease (GLILD) vs. Sarcoidosis

F Hamadeh, D Culver, C Farver. Cleveland Clinic, Cleveland, $\mathrm{OH}$.

Background: Patients referred to our Sarcoidosis Center receive an alternate diagnosis in $17 \%$ of the cases after the pathology is reviewed. One such alternative diagnosis is granulomatous-lymphocytic interstitial lung disease (GLILD). This is an interstitial lung disease (ILD) that has been described in a subset of patients with common variable immunodeficiency (CVID) and has pathologic features that can overlap significantly with those of sarcoidosis. We conducted a histopathologic comparison between a group of patients with documented GLILD arising in the setting of CVID and a matched group of patients with sarcoidosis.

Design: Cases histologically and clinically suspicious for GLILD were reviewed from the authors' case files. 8 cases consistent with GLILD and 8 cases of pulmonary sarcoidosis were identified and $\mathrm{H} \& \mathrm{E}$ slides from pulmonary specimens were reviewed. Tissue stains for microorganisms (Grocott methenamine silver -GMS- for fungus and Ziehl-Neelsen acid fast -ZN/AFB- for mycobacteria), immunohistochemical stains (CD3, CD4, CD8, CD20 and HHV-8) as well as in situ hybridization for EBV were performed. Whole slide imaging (WSI, Aperio AT scanner) and a Positive Pixel Count Aperio Algorithm were used to estimate the percentage of tissue $\mathrm{B}$ and $\mathrm{T}$ lymphocyte and CD4 and CD8 T lymphocytes in GLILD and sarcoidosis.

Results: Pulmonary tissue sections from GLILD cases showed more prominent interstitial inflammation with increased areas of organizing pneumonia when compared to the pulmonary sarcoidosis cases. In addition, the granulomas in both GLILD and sarcoidosis cases were non-necrotizing. However, the GLILD granulomas were not in a lymphatic distribution as seen in sarcoidosis, and were more loosely formed. Special stains for microorganisms (GMS and ZN-AFB), immunohistochemistry for HHV-8 and in situ hybridization for EBV were negative in both groups. The difference in tissue CD3:CD20 lymphocyte ratio and the CD4:CD8 ratio between the two groups was not statistically significant

Conclusions: The histopathologic features that distinguish GLILD from sarcoidosis include a brisk interstitial inflammatory infiltrate with areas of organizing pneumonia and more loosely formed non-necrotizing granulomas. Immunohistochemical stains for $\mathrm{B}$ and $\mathrm{T}$ lymphocytes and their subsets reveal no significant difference in the ratio of these lymphocytes in GLILD and sarcoidosis.

1967 p16 FISH in Malignant Pleural Mesothelioma: Both Homozygous and Heterozygous Deletions Have Diagnostic Utility

$M$ Hamasaki, S Matsumoto, T Kamei, K Hiroshima, K Kawahara, T Tsujimura, A Iwasaki, K Nabeshima. Fukuoka University Hospital, Fukuoka, Japan; Yamaguchi Grand Medical Center, Hofu, Japan; Tokyo Women's Medical University, Yachiyo Medical Center, Yachiyo, Japan; Osaka Prefectural Medical Center for Respiratory and Allergic Disease, Habikino, Japan; Hyogo College of Medicine, Hyogo, Japan. Background: Homozygous deletion (homo-d) of 9p21 assessed by fluorescence in situ hybridization (FISH) is useful for differentiating malignant pleural mesothelioma (MPM) from reactive mesothelial hyperplasia (RMH). Both heterozygous deletion (hetero-d) and homo-d are observed in every tissue examined; however, few reports describe cutoff values for the two deletions. Moreover, the role of hetero-d in MPM diagnosis is unclear. Here, we assessed homo-d and hetero-d cutoff values and investigated the roles of these deletions in MPM diagnosis.

Design: We performed 9p21 FISH using 190-kb Vysis or 110-kb GSP probes in 83MPM and 47 RMH samples. Cutoff values were evaluated for homo-d and hetero-d based on 9p21 FISH results for RMH. p16 gene copy number was analyzed by real-time PCR in $18 \mathrm{MPM}$ and $17 \mathrm{RMH}$ for which DNA was available. Overall survival was evaluated using Kaplan-Meier survival analysis and logrank test.

Results: Cutoff values were set at $10 \%$ and $47 \%$ for homo-d and hetero-d, respectively. In MPM, 74/83 (89\%) were homo-d positive, and 18/83 (22\%) were hetero-d positive. All 18 hetero-d-positive MPM were also positive for homo-d. No RMH was homo/ hetero-d positive. In 7 of the 18 hetero-d positive MPM, 9p21FISH with the GSP probe showed more homo-d and less hetero-d compared with the longer Vysis probe. Real-time PCR showed two allele p16 loss in 14 homo-d-dominant (by FISH) MPM. Among four hetero-d-dominant MPM samples, PCR revealed that half had one deleted p16 allele and half had two deleted alleles. No promoter methylation was found in the two cases with single allele deletion. Follow-up data was available in 25 MPM cases. Overall survival was significantly shorter in homo-d-positive MPM $(n=21)$ than homod-negative MPM $(n=4, p=0.01)$. Hetero-d-dominant MPM showed similar survival to homo-d dominant MPM.

Conclusions: 9p21 FISH is very useful for diagnosing MPM using cutoff values. We found that the presence of homo-d was specific to MPM and correlated with poor prognosis. Additionally, it is useful to perform PCR in hetero-d-dominant MPM to confirm homo-d of the p16 gene. There may be a p16-independent tumorgenesis pathway in these MPM cases. 
1968 Malignant Mesothelioma: Immunohistochemical Study of Mismatch Repair Proteins (MLH1, PMS2, MSH2, MSH6) with Clinicopathologic Correlation

D Hamatake, D Chen, D Shimizu, P Tauchi-Nishi. Fukuoka University, Fukuoka, Japan; University of Hawaii/The Queens Medical Center, Honolulu, HI.

Background: Albeit a rare tumor, malignant mesothelioma (MM) is increasing in incidence worldwide. Asbestos exposure imparts a 1000-fold elevated predilection. Other risk factors include exposure to the SV40 virus, as well as genetic abnormalities such as the BAP-1 mutation and homozygous 9p21 deletion. Recently, a 56 year old patient with peritoneal mesothelioma and Lynch syndrome was reported in the literature. Additionally, the patient had right-sided colon adenocarcinoma and multiple skin and nasal sebaceous carcinomas. His family history was positive for $1^{\text {st }}$ degree relatives with colon cancer. His mesothelioma exhibited loss of mismatch repair (MMR) proteins MLH1 and PMS2 by immunohistochemical (IHC) staining. Mutational analysis revealed a deleterious germ-line mutation in MLH1. In this study, our intent was to determine the prevalence of MMR protein loss in our mesothelioma patient population. We also aspired to correlate these results with relevant clinicopathologic features.

Design: From January 1991 to April 2013, we identified 37 patients with malignant mesothelioma in our database. Their average age was 67 (range 45-88). There was a predominance of males $(33,89 \%)$. Thirty-two $(86 \%)$ of these tumors originated in the pleura, whereas $5(14 \%)$ were peritoneal. Twenty-six $(70 \%)$ were of epithelioid histology, and $10(27 \%)$ sarcomatoid. Their slides were reviewed, and tissue microarray blocks were constructed. IHC staining with antibodies against MLH1, PMS2, MSH2, and MSH6 was performed, and then confirmed on pertinent whole slide sections. Results: All 37 of our mesothelioma cases retained MSH2 and MSH6 staining. Two (5.4\%) of the cases demonstrated loss of MLH1 and PMS2 expression (Table 1). Both patients were 64 year old males with pleural epithelioid mesotheliomas. They had no additional malignancies and no family histories of cancer.

MMR Loss in Mesothelioma Patients

\begin{tabular}{|c|c|c|c|c|c|c|c|}
\hline Age & Sex & Ethnicity & Occupation & $\begin{array}{l}\text { Tumor } \\
\text { Location }\end{array}$ & $\begin{array}{l}\begin{array}{l}\text { Histologic } \\
\text { Type }\end{array} \\
\text { y. }\end{array}$ & \begin{tabular}{|l} 
MMR \\
Loss
\end{tabular} & $\begin{array}{l}\text { Family Hx or } \\
\text { Additional Patient } \\
\text { Cancer }\end{array}$ \\
\hline 64 & M & Caucasian & pilot, teacher & pleura & epithelioid & $\begin{array}{l}\text { MLH1, } \\
\text { PMS2 }\end{array}$ & none \\
\hline 64 & M & Chinese & $\begin{array}{l}\text { naval shipyard } \\
\text { worker }\end{array}$ & pleura & ithelioid & $\begin{array}{l}\text { MLH1, } \\
\text { PMS2 }\end{array}$ & none \\
\hline
\end{tabular}

Conclusions: To our knowledge, this is the 1st such study of MMR protein loss in malignant mesotheliomas by IHC staining. Absence of MLH1 and PMS2 expression was observed in only $5.4 \%$ of our patients. No correlations were observed between MMR protein loss and clinicopathologic features.

1969 Quantification of ALK from Formalin-Fixed Paraffin-Embedded Non-Small Cell Lung Cancer Tissue by Mass Spectrometry

CP Hartley, W-L Liao, J Burrows, T Hembrough, LJ Tafe. Geisel School of Medicine at Dartmouth and Dartmouth-Hitchcock Medical Center, Lebanon, NH; OncoPlex Diagnostics, Rockville, MD.

Background: $A L K$ rearrangement leads to constitutive activation of the ALK kinase domain and is seen in $2-5 \%$ of NSCLC. Crizotinib has significant anti-tumor activity in $A L K$ rearranged (AR) NSCLC and the FDA approved break-apart FISH test is commonly used to determine treatment eligibility. However, $A L K$ FISH is laborious, expensive, and challenging to interpret. Although ALK is not normally present in lung, in AR tumors the ALK kinase domain is detectable and may be used to identify these tumors. The "LungAdeno-plex" Liquid-Tissue-selected reaction monitoring (LT-SRM) MS test is a mass spectrometry proteomic assay developed to generate quantitative data in a single run, for multiple proteins biomarkers, such as TTF1, K7, p63, K5, EGFR, MET, KRAS and ROS1 in NSCLC. In the current study, we sought to develop a quantitative ALK assay within the LungAdeno-plex MS test and correlate the findings with $A L K$ FISH results.

Design: We used trypsin digestion mapping of recombinant ALK (rALK) to identify unique peptides for MS assay development. Stable isotope-labeled peptides were synthesized as internal standards, and standard curves were generated in pyrococcus complex matrix to determine LOD, LLOQ, accuracy, precision and linearity of the assays. The assay was pre-clinically validated in an $E M L 4-A L K$ rearrangement positive NSCLC cell line-H3122. The assay was run on 11 archived FFPE sections from 10 cases ( 8 adenocarcinomas and 2 adenosquamous carcinomas) where corresponding $A L K$ FISH status was known.

Results: Peptides SNQEVLEFVTSGGR (SNQ) and DPEGVPPLLVSQQAK (DPE) of the ALK kinase domain were found optimal for ALK quantification. The LLOD were 150 amol and 100 amol, respectively, with CV $<20 \%$. ALK positive cells (H3122) expressed 396amol ALK/ug cell protein. ALK peptides were not detected in AR negative control NSCLC tissues or in one known AR case; the remaining 6 AR NSCLC clinical samples expressed levels of ALK from 118 to $437 \mathrm{amol} / \mathrm{ug}$ based on DEP peptide, and not detectable to 354amol/ug based on SNQ. The data show that the protein concentrations in AR NSCLC were relatively low and that when the protein level approached 100amol/ ug, only DEP peptide was detected.

Conclusions: The LungAdeno-plex ALK assay was able to detect ALK protein in $86 \%$ of the AR samples tested. One application is screening for the ALK protein in NSCLC; positive cases would be reflexed to FISH for confirmation. Additional studies are needed to validate the clinical performance of this assay.
1970 Detection of High-Risk Human Papilloma Virus Genotypes and P16 Up-Regulation in Small Cell Lung Cancer

CP Hartley, HB Steinmetz, VA Memoli, LJ Tafe. Dartmouth-Hitchcock Medical Center, Lebanon, NH.

Background: High-risk subtypes of the human papillomavirus (HR-HPV) are known to drive the pathogenesis of cervical, anogenital and oropharyngeal squamous cell carcinomas. Recent reports have shown that HPV is also associated with small cell neuroendocrine carcinomas of the cervix and nasopharynx. Little is known about HPV as a driver of neuroendocrine tumors at other sites, in particular, small cell lung cancer (SCLC). Almost all research on the association of HPV and primary lung cancers has focused on non-small cell lung carcinoma (NSCLC). The few studies that have included a small number of SCLC in evaluation of HPV report a wide range of positivity (0$100 \%$ ) by PCR and ISH. The aim of this study is to evaluate SCLC for the presence of HR-HPV genotypes and p16 expression to further elucidate the role of HPV in SCLC. Design: Archived formalin-fixed, paraffin-embedded (FFPE) surgical resection specimens from 14 primary small cell lung cancers were identified from 2005-2013. The specimens included 8 lobectomies and 6 wedge resections. Small cell neuroendocrine phenotype was confirmed by review of morphology and prior IHC staining. IHC for p16 (INK4a) expression was performed in all cases. DNA was extracted from FFPE and run on the Roche Linear Array HPV Genotyping test, a PCR based line-blot assay that is currently run in our clinical laboratory. This test detects 37 high and low risk human papillomavirus genotypes. Pathologic tumor stage was collected from review of the surgical pathology reports.

Results: Eleven samples were negative for HR-HPV by the genotyping assay; the extracted DNA from the remaining three cases was not suitable for amplification. Thirteen of 14 SCLC showed strong and diffuse nuclear and cytoplasmic p16 overexpression; one was negative. The pTNM stages of the tumors were: IA (5), IB (2), IIB (2), IIIA (4), and IV (1).

Conclusions: HR-HPV genotypes were not detected in any of the 11 SCLC specimens successfully tested, while p16 was up-regulated in 13 of 14 (93\%). p16 up-regulation can be used as a surrogate marker of HPV genome integration due to Rb pathway interruption by HPV oncoprotein E7. However, the Rb pathway is known to be inactivated by other mechanisms in SCLC such as $\mathrm{p} 53$ mutations or $17 \mathrm{p}$ deletions also leading to up-regulation of $\mathrm{p} 16$. In conclusion, our findings indicate that unlike some other small cell neuroendocrine carcinomas, the pathogenesis of SCLC does not appear to be associated with HR-HPV infection.

1971 Intra-Tumor EGFR Mutation Status Heterogeneity in Lung Adenocarcinoma Is Associated with Reduced Surviva

D Hershkovitz, E Simon, E Prinz, T Shentzer, L Chekinsky, E Sabo, O Ben-Izhak. Rambam Health Care Campus, Haifa, Israel; Technion - Israel Institute of Technology, Haifa, Israel.

Background: Current EGFR mutation detection methods in the management of lung adenocarcinoma indicate the presence or absence of specific mutations; however, none of these methods identify variables such as intra-tumor heterogeneity (ITH) and mutated allele imbalance (MAI), which might have potential relevance to treatment decisions and prognosis. In the present report we describe a molecular morphometric approach that can identify MAI and ITH in clinical samples. This approach was utilized on a subset of lung adenocarcinoma cases.

Design: DNA was extracted from 314 surgical specimens of lung adenocarcinomas and was scrutinized for EGFR mutation. Thirty EGFR mutation positive cases, presented herein, underwent deep sequencing to determine the relative fraction of mutated EGFR copies. Additionally, for each case, a pan-cytokeratin stained slide was scanned at high resolution and analyzed by a pathologist with the assistance of computerized morphometry software to determine the fraction of tumor cells in the area used for DNA extraction. Tumor cells fraction and the fraction of mutated DNA copies were used to identify the presence of ITH or MAI, which were further correlated with patients' clinical and histopathological variables.

Results: Of the 314 cases analyzed, 100 screened positive for EGFR mutations. In the 30 EGFR mutation positive cases presented herein, the median tumor cell fraction in the areas used for DNA extraction was $43 \%(7-78 \%)$ and the median fraction of mutated DNA copies was $34 \%(8-96 \%)$. Four cases $(13 \%)$ showed ITH determined by the presence of less mutated DNA copies than expected from the tumor cell fraction analysis. Ten cases $(33 \%)$ showed mutated allele copy gains of up to 88 fold. Significantly reduced survival times were noticed in the patients with ITH group with only $50 \%$ of the patients surviving longer than 30 months from diagnosis (median survival time: 30) compared to the other patients ( $89 \%$ surviving longer than 43 months, median survival time has not been reached), $\mathrm{P}=0.02$ (Log-Rank test). The presence of ITH was not associated with any of the other clinical or pathological variables including disease stage and histological types.

Conclusions: A combined molecular morphometric approach can help identify the presence of ITH and MAI in the clinical setting. In our sample set, ITH was associated with reduced survival time. The role of ITH in treatment decisions and prognosis should be further evaluated in larger cohorts.

1972 Manual and Laser Capture Microdissection: Optimizing EGFR Molecular Testing in Small Biopsies and Cytology Specimens

JJ Heymann, B Singh, BH Durham, MM Mansukhani, JP Crapanzano, A Saqi. Columbia University Medical Center, New York, NY.

Background: Lung cancer is the leading cause of cancer death worldwide. Patients with lung adenocarcinoma (ADCA) harboring epidermal growth factor receptor (EGFR) mutation may respond to targeted tyrosine kinase inhibitor (TKI) therapy. ADCA diagnosis is often rendered at an advanced stage and on small biopsies (BXs) 
and cytology specimens (CYs). Such small specimens (SSs) may provide the only tissue for mutation testing and determination of TKI therapy eligibility. Recently published guidelines recommend EGFR testing in all SSs that potentially harbor ADCA and contain $50 \%$ tumor cell content with a minimum of 200-400 tumor cells. Achieving 50\% content in SSs may be hindered by adjacent non-tumor cells such as lymphocytes in lymph node (LN) metastases. The aim of the current study was to assess EGFR molecular testing on SSs with an optimized algorithm utilizing various methods of tissue microdissection. Design: A one-year retrospective search for SSs containing lung ADCA was performed. Reflex EGFR mutational analysis was performed on formalin-fixed paraffin-embedded CYs [fine needle aspirations (FNAs) and body fluid (BF) cell blocks] and BXs (core needle and endoscopic biopsies). Exons 18-21 were amplified by polymerase chain reaction (PCR) to detect EGFR mutation following microdissection by a pathologist. Manual microdissection (MD) at 100x with an 18-gauge needle or a \#10-\#11 scalpel blade was performed for specimens with moderate-marked and minimal-mild background inflammatory cells, respectively. For specimens devoid of tumor cell clusters (single tumor cells only), laser capture microdissection (LCM) was employed. Results: One hundred twenty-six samples $(\mathrm{FNA}=47, \mathrm{BF}=14, \mathrm{BX}=65)$ from lung $(\mathrm{n}=61), \mathrm{LN}(\mathrm{n}=29)$, pleura $(\mathrm{n}=14)$, and distant sites $(\mathrm{n}=22)$ were sampled. Tissue was microdissected using LCM $(\mathrm{n}=16)$ or MD (scalpel $=25$, needle $=85$ ). LCM was used more frequently for CYs than BXs $(21 \%$ vs. $6 \%, \mathrm{p}=0.01)$. EGFR mutation analysis yielded interpretable results in 124 samples ( $98 \%$ ), including 30 positive results $(24 \%)$. Both cases in which EGFR mutation analysis failed were FNAs of lung masses - one underwent scalpel MD and the other LCM. There was no significant difference in EGFR mutation detection rate among cases that underwent MD with a blade/needle or LCM ( $22 \%$ vs. $24 \%$ vs. $29 \%$, $\mathrm{p}=0.48)$; this is similar to reported EGFR mutation rates. Conclusions: A 98\% success rate for EGFR mutation analysis on SSs is achievable through the use of microdissection with minimal reliance on the more costly and timeconsuming method of LCM, especially in tissue biopsies.

1973 The Use of a Novel Immunohistochemical Triple Cocktail in the Subclassification of Non-Small Cell Lung Carcinoma (NSCLC)

K Hoo Kim, CS Chaudoir, MD Reid, SI Chaudhery. LSUHSC-Shreveport, Shreveport, LA; Emory University School of Medicine, Atlanta, GA.

Background: Accurate sub-classification of NSCLC is essential for therapy and performance of appropriate molecular studies. While Napsin-A, TTF1, and p40 have been used individually in the subtyping of NSCLC, the 3 have never been studied in cocktail form. We evaluated the utility of a novel, triple cocktail (comprised of TTF1, Napsin-A, p40 [TNP]) in the subclassification of 36 resected NSCLC.

Design: A retrospective review of previously diagnosed NSCLC resections yielded 36 total cases which were randomly sorted and double-blinded before review. Clinicopathologic data were also examined. The triple antibody cocktail (brown nuclear stain, TTF; red nuclear stain, p40; and red granular cytoplasmic stain, Napsin-A) was applied to a single representative tumor slide. 2 reviewers $(\mathrm{KH}, \mathrm{SC})$ independently confirmed staining and classified tumors. Discrepant cases were stained separately with p63, CK5/6 and TTF-1.

Results: 29 of 36 cases $(80.6 \%)$ showed correlation with original diagnosis; 16 of 18 $(89 \%)$ adenocarcinomas (ADC) were TTF/Napsin-A positive (+); 2 (11\%) mucinous ADC were TTF/Napsin-A negative (-); 13 of 14 squamous cell carcinomas (SCC) were $\mathrm{p} 40(+)$; and $1 \mathrm{SCC}$ was $\mathrm{p} 40(-)$ but $\mathrm{p} 63(+)$. No ADC was misdiagnosed as SCC and vice versa. Four $(11 \%)$ of the original NSCLC cases were reclassified as large cell carcinoma (3) and SCC (1).

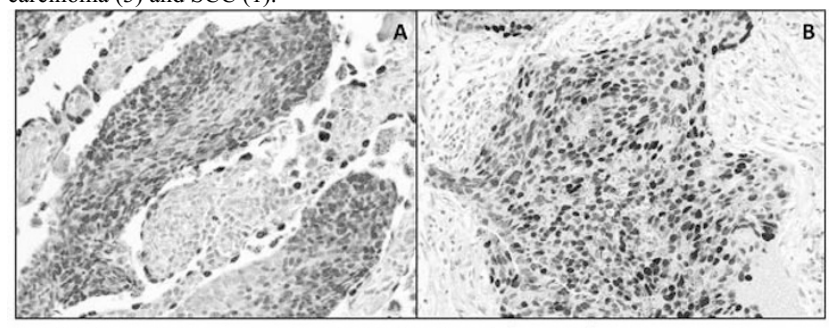

Figure 2. (A) Invasive squamous cell carcinoma (20X) stained p40+/Napsin-A-/TFF-1.. Note presence of positive internal controls on same slide for Napsin-A (alveolar macrophages, red granular cytoplasmic staining) and for TF-1 (pneumocytes, brown nuclear staining):

(B) Adenocarcinoma (20X) with co-expression for TTF-1 and Napsin-A (TTF-1+/Napsin-A+/p40-).

Diagnostic Assessments of NSCLC

\begin{tabular}{|c|c|c|}
\hline 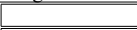 & Resection diagnosis** & TNP classification** \\
\hline ADC & 13 & 16 \\
\hline SCC & 11 & 13 \\
\hline NSCLC, NOS & 12 & $7 *$ \\
\hline
\end{tabular}

ADC, adenocarcinoma; SCC, squamous cell carcinoma; NSCLC, nonsmall cell lung carcinoma, TNP, TTF1/Napsin-A/p40; * includes 2 TNP(-) ADC and 1 TNP(-) SCC; ** independent assessment by 2 reviewers (KH, SC)

Conclusions: The novel TNP triple antibody cocktail accurately subclassifies NSCLCs in the majority ( $92 \%$ ) of cases and requires only a single slide for preparation. This methodology may improve tissue availability for molecular testing without sacrificing diagnostic accuracy.
1974 Next Generation Sequence Profiling of Large Cell Carcinoma/ Solid Adenocarcinoma Defines Distinct Subgroups with Implications for Rational Therapy Selection

DH Hwang, Y Jia, E Garcia, A Perry, F Kuo, LM Sholl. Brigham and Women's Hospital, Bost, MA; Utah Valley Regional Medical Center, Provo, UT.

Background: Large cell carcinoma (LCC) lacking squamous or neuroendocrine differentiation is clinicopathologically indistinguishable from solid adenocarcinoma (ADC) and is frequently grouped in the ADC subgroup for the purposes of molecular triage and therapy. LCC/solid ADC is strongly associated with a smoking history and harbors a high ( $40 \%)$ frequency of $K R A S$ mutations. The majority of LCC/solid ADC, however, lack one of the commonly queried oncogenic driver alterations (including $E G F R, A L K, K R A S$ ), thus therapeutic options are limited for patients with this tumor type. This study aims to clarify the mutational spectrum of $\mathrm{LCC} /$ solid ADC and to determine the frequency of possible therapeutic targets.

Design: Targeted next generation sequencing (NGS) was performed using a cancer genomic assay to detect mutations, copy number variations (CNVs) and structural variants. The assay captures exonic sequences of 275 cancer genes and 91 introns across 30 genes for rearrangement detection. DNA was isolated from formalin fixed paraffin embedded whole tissue sections containing at least $20 \%$ tumor nuclei and analyzed by massively parallel sequencing using a solution-phase Agilent SureSelect hybrid capture kit and an Illumina HiSeq 2500 sequencer. MuTect and GATK (Broad Institute) were used to detect single nucleotide variants and indels. VISCAPCancer and CCOPY were used to detect CNVs.

Results: $18 \mathrm{LCC} /$ solid ADC known to be $K R A S, E G F R$ and $A L K$ wild type were studied. The median number of mutational events was 10 (range 2-65). On average, $79 \%$ of mutations were transversions. 17/18 (94\%) cases had TP53 mutations or copy loss. $44 \%$ of cases had a clinically targetable oncogenic alteration including $\operatorname{HRAS}(\mathrm{n}=1)$, NRAS (n=1), PIK3CA mutations ( $\mathrm{n}=3)$ and MET amplification $(\mathrm{n}=3) .5 / 18(28 \%)$ of cases contained deleterious alterations in $S T K 11$. Four highly mutated tumors $(\geq 20$ substitution variants) lacked common alterations other than TP53 mutations.

Conclusions: Consistent with prior reports, this smoking-related lung tumor type contains a high frequency of transversion mutations. MAPK, PI3K, MEK, and MET pathway alterations are common, suggesting that patients with wild type LCC/solid ADC by standard clinical testing practices may be candidates for ongoing trials of MEK, PI3K and MET inhibitors. A subset of LCC/solid ADC have an unusually high mutation rate; further investigation to identify a unifying mechanism such as a DNA repair defect is needed.

1975 MYC Protein Expression Correlates with MYC Amplification in Small Cell Lung Carcinoma

DH Hwang, H Sun, SJ Rodig, JL Hornick, LM Sholl. Brigham and Women's Hospital, Boston, MA.

Background: MYC family members are important contributors to oncogenesis in a variety of tumor types. Small cell lung carcinoma (SCLC), when diagnosed at an advanced stage, is rapidly and uniformly fatal. Treatment options are limited, and the identification of therapeutic targets for this tumor type is needed. Next generation sequencing-based studies of have identified $N-M Y C, M Y C L 1$, and MYC amplification in 8,6 and $\%$ of SCLC, respectively [Peifer et al., 2012]. MYC protein expression was recently demonstrated to reflect underlying MYC pathway activation in lymphomas. Thus, we hypothesize that immunohistochemistry (IHC) for MYC protein overexpression could identify SCLC with MYC pathway activation and that this expression would correlate with gene amplification.

Design: 51 cases of SCLC were retrieved from departmental archives between the years 1999 and 2013. Diagnoses were confirmed following review of H\&E and IHC for synaptophysin, chromogranin, and Mib-1 if needed. IHC for MYC (clone Y69, Abcam) was performed with nuclear staining scored as follows: 0 absent, $1+(<25 \%$ of cells positive), $2(25 \%$ to $75 \%), 3(>75 \%)$. MYC copy number $(\mathrm{CN})$ was assessed by dual color chromogenic in situ hybridization using probes to the MYC locus and Chr8 (Ventana Medical Systems, Inc., Tucson, AZ). MYC CN was scored by evaluating 50 cells for signals/cell for both the $M Y C$ locus and Chr8 probes. Cases were classified as $\mathrm{CN}$ gain for scores of $3 M Y C$ signals/cell or more, and as amplified for scores of 6 signals/cell or more.

Results: MYC protein expression was absent in $19,1+$ in $9,2+$ in 2 , and $3+$ in 5 cases. The number of CISH MYC signals per cell ranged from 1.5 to $>15$, with average counts as follows: IHC negative (2.61), score 1 (2.23), IHC score 2 (3.63), IHC score 3 (9.72). 18 cases failed CISH analysis, typically due to crush artifact. CISH for MYC $\mathrm{CN}$ correlated with IHC for MYC protein expression (Spearman's $\rho=0.41, \mathrm{p}=0.02$ ). MYC copy gain, including due to polysomy of $\mathrm{Chr} 8$, was seen in $10 / 33(30 \%)$ cases by CISH. Amplification, present as either homogenously staining regions or double minutes, was seen in $3 / 33(9 \%)$ cases.

Conclusions: By in situ hybridization, MYC amplification was identified in 9\% of SCLC and correlated with strong protein expression. This frequency is higher than previously reported and may reflect the tendency of sequencing-based assays to underestimate copy number changes due to benign stromal contamination and tumoral heterogeneity. As novel MYC- targeted therapies develop, CISH and IHC should be considered as tools for identification of MYC pathway dysregulation. 
1976

EGFR Mutation Testing in Lung Adenocarcinoma Patients Using

\section{Sequenom Assau}

J Iqbal, KL Lim, SK Sai, E Chen Xueqin, L Oon, LK Hon, D Tan. Singapore General Hospital, Singapore, Singapore; National Cancer Center Singapore, Singapore, Singapore.

Background: Epidermal growth factor receptor (EGFR) mutation analysis by Sanger sequencing is part of routine clinical practice. However, single-base primer extension and analysis of extension products by matrix-assisted laser desorption/ionization-time of flight mass spectrometry (MALDI-TOF) or Sequenom assay (SA) has been used for somatic mutation analyses. Our objective was to assess suitability of SA to detect EGFR mutation in formalin fixed paraffin embedded (FFPE) material and cytology samples of lung adenocarcinoma patients compared to widely used Sanger sequencing (SS).

Design: DNA was extracted from 31 archival excision FFPE samples and 17 cytology samples with known EGFR direct Sanger Sequencing results. Six of cytology cases had concurrent biopsy tissue. Cellblocks were prepared from the remaining 11 cases. Five and 10 sections $(10 \mu \mathrm{m})$ were cut from cell blocks and biopsy tissue respectively for DNA extraction and subjected to Sanger sequencing. DNA sample concentration and purity were measured using the Nanodrop 2000 spectrophotometerEGFR mutation studies were performed using Sequenom MassArray System (OncoCarta ${ }^{\text {TMPanel v1.0). }}$ Results: From the 31 excision samples, 26 had concordant results on the Sequenom OncoCarta v1.0 panel: 12 concordant samples were positive for EGFR E746 A750del and 11 concordant samples were positive for EGFR L858R. One concordant sample showed both EGFR L747_S752del and T790M mutations. Two samples were negative on both SS and SA. Of the discordant group, two cases positive for EGFR mutations by SS were negative on SA. Three cases negative on SS showed one EGFR mutation and two K-ras mutations on SA. Interestingly, 25 samples showed multiple mutations on SA. In the cytology group, concordance rate was $\sim 90 \%$. EGFR mutations were detected in 6/11 cases with SS from DNA extracted from cell blocks. Using ThinPrep DNA, mutations were detected in $4 / 6$ cases by SA in this group. Two of six cases with concurrent biopsy tissue showed EGFR mutation by SA were confirmed on the ThinPrep remnant DNA using SA. The two samples that were negative on SA lacked specific primers for the corresponding mutations detected in SS. However, SA identified several non-EGFR mutations (K-ras) in these samples.

Conclusions: Our study demonstrates the feasibility of Sequenom assays in EGFR mutation analyses in both FFPE and cytology specimens. Due to ability of this platform to profile multiple somatic mutations in parallel, use of Sequenom may be considered in high volume laboratories.

1977 Clinical Genotyping of Non-Small Cell Lung Cancers Using Semiconductor Next Generation Sequencing Technology

EG Jenson, JD Peterson, F de Abreu, TL Gallagher, JR Rigas, KH Dragnev, CI Amos, GJ Tsongalis, LJ Tafe. Geisel School of Medicine at Dartmouth and Dartmouth-Hitchcock Medical Center, Lebanon, $\mathrm{NH}$

Background: Genotyping of non-small cell lung cancers (NSCLC) is important for directing patient care, particularly in adenocarcinomas where targeted therapeutics are available. It is now possible to test individual tumors simultaneously for somatic mutations in multiple genes using next-generation sequencing technologies. Here, we present our experience with genotyping NSCLC using the Ion Torrent PGM and the AmpliSeq Cancer Hotspot Panel v2 since adopting this into clinical practice in late May 2013

Design: To date, 78 NSCLC cases ( 50 cytology specimens, 14 small biopsies, and 14 excisions) have been tested using the 50 gene AmpliSeq Cancer Hotspot Panel v2. Briefly, DNA was extracted from unstained FFPE slides with a minimum tumor cellularity of $10 \%$. DNA was quantified using the PicoGreen method. Barcoded libraries were prepared using up to 10ng of DNA; sequencing was performed on the Ion Torrent PGM (318 chip). Variants were identified using the Ion Torrent Variant Caller Plugin (v3.6.59049) and reference genome hg19. Golden Helix's SVS software v7.7.3 was used for annotation of the variants, as well as prediction of the significance of the variants. Results: Up to 15 samples were run simultaneously on the 318 chip. The PGM provided a minimum of $100 x$ coverage with the majority of amplicons having $>500 x$ coverage. All variant calls were initially filtered to remove non-coding and synonymous mutations. Golden Helix was then used to annotate variants and help predict pathogenicity. All reported variants received sufficient coverage and were of high enough frequency to be annotated as true variants. Only 3 cytology samples failed QC due to low DNA concentrations. Of the remaining 75 samples, 14 were wild-type, 31 had one and, 30 had two or more clinically relevant mutations. The most frequent mutations identified were in the following genes ( $\%$ of cases with mutation): TP53 (33\%), KRAS (24\%), EGFR (20\%), STK11 (9\%) and MET (8\%). Mutations in 17 additional genes were seen in 1-3 cases, each.

Conclusions: The Ion Torrent Ampliseq technology can be successfully performed on small biopsy and cytology specimens, requires very little input DNA, and can be readily applied in clinical laboratories for genotyping of NSCLC.

1978 The Frequency of Chromatin Regulator Disruption in Squamous Cell Carcinoma of the Lung and Role for a Distinct Molecular Pathway in Tumorigenesis

SE Johnstone, A Chi, E Mark, M Mino-Kenudson. Massachusetts General Hospital, Boston, MA.

Background: Genetic alterations of chromatin remodeling subunits have been widely reported in a variety of cancers and are thought to mediate tumorigenesis through mis-regulation of gene expression networks. Arid1 A and Brg1, two well characterized chromatin remodeling subunits, have been reported to be mutated in multiple tumor types, however the exact incidence of their disruption in squamous cell carcinoma (SqCC) of the lung is not clear. It is also not understood how these mutations relate to other classical genetic alterations in $\mathrm{SqCC}$ of the lung.

Design: A prospective database of resected lung cancers was compiled of patients with $\mathrm{SqCC}$ who underwent primary resection without neoadjuvant therapy between 2005 and 2010. Of those, cases with available tissue blocks $(n=97)$ formed our study cohort. Immunohistochemical stains for Arid1A, and Brg1 were performed on tissue microarrays representing these cases. These data were then compared with clinical data including survival and genetic data querying 84 genes that was obtained as part of routine clinical care, and the results of PTEN expression examined on the tissue microarrays. Results: A small subset ( 8 of 97 cases) of SqCC lost either expression of Arid1A or Brg1. No case harbored loss of both factors. Loss of either Arid1 A or Brg1 in SqCC is associated with a later stage presentation and worse 5 year survival compared to no loss $(23 \%$ vs $72 \% \mathrm{p}=0.192)$, however, the difference did not reach a statistical significance likely secondary to small sample size. Also, in cases with loss of Arid1A or Brg1, no mutation in PIBK or $A K T$, or loss of PTEN expression was observed.

Conclusions: While only a small subset of SqCC cases harbor loss of Arid1A or Brg1, this group appears to have unique clinical behavior in terms of clinical presentation and survival. Additionally, the data presented here suggest that Aridla and Brg1 may be acting through a distinct biological pathway since their disruption does not occur in tumors carrying PI3K/AKT pathway alterations. Further exploration of the role of these factors in SqCC is warranted given both their prognostic and biological potential.

\section{Small Airway Centered Granulomatosis Occurred in} Polytetrafluoroethylene Workers

HR Jung, W-I Choi, E Shehu, BH Rho, M-Y Lee, SS Lee, SP Kim, YN Kang, M Choe, SY Kwon, I Hwang, JY Park, KY Kwon. Keimyung University School of Medicine, Daegu, Republic of Korea; Regional Hospital of Durres, Durres, Albania; Kyungpook University, Daegu, Republic of Korea.

Background: Polytetrafluoroethylene (PTFE, Teflon) is a widely-used, chemically stable fluorocarbon polymer. Pyrolysis products of PTFE have induced "polymer fume fever" in the industrial workers. To date, there have been no reports of chronic pulmonary granulomatosis associated with long termexposure to PTFE

Design: We report 3 cases of small airways centered granulomatous lesions in workers who had employed at facilities that apply coatings to pans and other utensils. The workers were repeatedly exposed to PTFE particles that were probably generated by the high-pressure air spray and high temperatures $\left(380-420^{\circ} \mathrm{C}\right)$ used in the application process. The duration of inhalational PTFE exposure was between 7 and 20 years. We performed light microscopy, electron microscopy, energy-dispersive $\mathrm{x}$-ray analysis (EDS), and chemical component analysis.

Results: We found granulomatous lung parenchymal lesions around the terminal and respiratory bronchioles in lung biopsy specimens obtained from the workers. Fourier transform infrared spectrometry analysis and scanning electron microscopy/energydispersive X-ray spectroscopy from the pulmonary tissue specimens identified PTFE by its chemical signature.

Conclusions: These cases suggest that recurrent inhalational exposure to PTFE particles causes small airways centered pulmonary granulomatosis.

1980 Poor Prognostic Driver Mutations in Early Stage Lung Adenocarcinomas (LADs): BRAF and KRAS Mutations for All LADs and KRAS Mutations within the Solid Subtype Alone

K Kadota, ME Arcila, C Hedvat, MG Kris, VW Rusch, PS Adusumilli, WD Travis. Memorial Sloan-Kettering Cancer Center, New York, NY.

Background: Studies of molecular histologic correlations are emerging according to the IASLC/ATS/ERS LAD classification; however few have been performed with comprehensive genotyping in large series of patients with long term followup. We investigated whether driver mutations correlate with histologic subtypes and prognosis in a large series of early stage LADs.

Design: In 482 early stage (stage I/II: 463/19) resected LADs (1995-2005, median followup duration: 4 yrs), driver mutations were assessed using Sequenom mass spectrometry genotyping and EGFR exon 19 deletions were identified by a PCR-based assay. Immunostaining was performed for ALK (5A4) using tissue microarrays. Tumors were subtyped according to the IASLC/ATS/ERS classification. Log-rank test was used for overall survival (OS).

Results: Correlations between mutations and histologic subtypes or OS are summarized in Table. Lepidic predominant pattern had equal numbers of $K R A S$ mutations and $E G F R$ exon $21 \mathrm{~L} 858 \mathrm{R}$ mutations but a significant association was only seen with the latter $(\mathrm{p}=0.006)$ and not with exon 19 deletion $(\mathrm{p}=0.62)$. More than half of invasive mucinous adenocarcinomas and colloid predominant tumors had KRAS mutations but they did not have any other mutations. Tumors with KRAS mutation showed worse OS than tumors that were negative for driver mutations and ALK (Mut-Neg) $(\mathrm{p}=0.001)$ or had EGFR L858R mutation $(\mathrm{p}=0.051)$ and exon 19 deletion $(\mathrm{p}=0.017)$. Within the solid predominant LADs ( $\mathrm{n}=60$ ), tumors with KRAS mutation showed worse OS than KRAS wild type $(\mathrm{p}=0.011)$. Tumors with $B R A F$ mutations showed worse $\mathrm{OS}$ than tumors that were Mut-Neg $(\mathrm{p}=0.008)$, had EGFR L858R mutation $(\mathrm{p}=0.009)$ and exon 19 deletion $(\mathrm{p}=0.003)$ although this finding was on the basis of a small number of cases $(\mathrm{n}=8)$ 


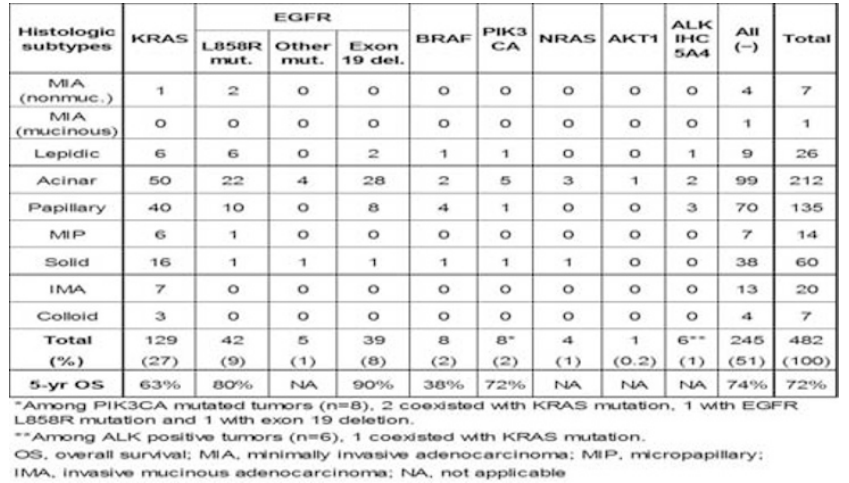

Conclusions: Our data suggest that driver mutations define molecular subtypes of early stage LADs with significantly different prognosis and $B R A F$ and $K R A S$ mutations are poor prognostic factors. A novel finding was that within the solid predominant LADs $K R A S$ mutation was a poor prognostic factor for OS.

1981 Micropapillary Pattern and Invasive Tumor Size Are Prognostic Factors in Invasive Mucinous Adenocarcinoma of the Lung

K Kadota, NRekhtman, AL Moreira, ME Arcila, VW Rusch, PS Adusumilli, WD Travis. Memorial Sloan-Kettering Cancer Center, New York, NY.

Background: According to the 2011 IASLC/ATS/ERS lung adenocarcinoma (ADC) classification, invasive $\mathrm{ADC}$ with prominent apical intracytoplasmic mucin and small basally oriented nuclei, formerly referred to as mucinous bronchioloalveolar carcinoma, is classified as invasive mucinous ADC (IMA). This classification emphasizes the prognostic significance of histologic subtypes. However, the prognostic impact of these subtypes in IMA is not known. In this study, we determine whether histologic patterns and tumor size can predict prognosis in IMA.

Design: All available tumor slides from patients with resected lung IMA (1995-2013) were reviewed ( $\mathrm{n}=93$; stage I/II/III: 74/16/3). The percentage of histologic pattern was recorded in $5 \%$ increments. Invasive tumor size was measured by subtracting the percentage of lepidic component from the total size. Tumors were classified according to the predominant subtype. Molecular testing ( $E G F R / K R A S$ mutation) was performed in 70 cases. Log-rank test was used to analyze recurrence-free probability (RFP).

Results: There were 3 mucinous minimally invasive (MIA) (2 pure mucinous and 1 mixed mucinous/nonmucinous) and none of them recurred. The RFP of all IMA ( $\mathrm{n}=90)$ was $79 \%$, which was between those of intermediate $(84 \%$ for acinar and $83 \%$ for papillary) and high grade (solid for 70\%) in non-mucinous tumors (Yoshizawa et al. Mod Pathol 2011). In IMA, 42 were pure mucinous and 48 mixed mucinous/nonmucinous, but no difference of RFP was observed $(\mathrm{p}=0.98)$. In tumors tested for mutations $(\mathrm{n}=70)$, no tumors had EGFR mutations but 34 had KRAS mutations, including 17 transversion and 17 transition. Pure mucinous tumors more frequently had KRAS mutations than mixed mucinous/nonmucinous $(70 \%$ vs. $30 \%$; $p=0.002)$. In tumors stained with the immunohistochemistry, $48 \%$ were positive for TTF- $1(47 \%$ in pure and $50 \%$ in mixed mucinous/nonmucinous), $95 \%$ positive for CK7, 56\% positive for CK20, and $6 \%$ positive for ALK (clone 5A4). By subtypes, 19 (21\%) lepidic, 31 (34\%) acinar, and $40(44 \%)$ papillary predominant tumors were identified, but no difference of RFP was observed $(\mathrm{p}=0.89)$. The RFP for patients with presence $(\geq 5 \%)$ of micropapillary pattern $(\mathrm{n}=30$; 5-year RFP: 52\%) was significantly lower than patients without micropapillary $(\mathrm{n}=63 ; 83 \% ; \mathrm{p}=0.009)$. Total tumor size did not correlate with prognosis $(\mathrm{p}=0.50)$. However, the RFP for patients with $>2 \mathrm{~cm}$ invasive size $(\mathrm{n}=37 ; 57 \%)$ was significantly lower than patients with $\leq 2 \mathrm{~cm}$ invasive size $(\mathrm{n}=56 ; 81 \% ; \mathrm{p}=0.016)$.

Conclusions: In IMA, invasive tumor size, but not total tumor size, and micropapillary pattern are significant predictors of recurrence. RFP for IMA appears intermediate to poor compared to other ADC subtypes. IMA, especially pure mucinous tumors, frequently had $K R A S$ mutations but no EGFR mutations.

1982 Is Peripheral Squamous Cell Carcinoma of the Lung a Different Neoplasm Than Central Squamous Cell Carcinoma? A Clinical, Pathologic and Epigenetic Study

JA Kalil, J Macagba Slade, L Buckingham, VB Reddy, A Medavaram, $R$ Ghai, $P$ Bitterman, $P$ Gattuso. Rush University Medical Center, Chicago, IL.

Background: Squamous cell carcinomas of the lung are categorized by tumor location: central and peripheral. Studies have addressed the clinical, pathologic and molecular characteristics of central squamous cell carcinoma (SCC-C) of the lung, but there is scant literature for peripheral (SCC-P). We undertook a retrospective study to compare the clinical, pathologic and molecular findings of peripheral and central SCC of the lung. Design: Between 1995 and 2011, 135 SCCs of the lung were retrieved from our pathology archives. Sixty-six cases were peripheral and 69 central. Clinical, pathologic and molecular studies (MGMT promoter methylation) were compared between the two groups.

Results: Among the 66 patients with SCC-P, 34 were female and 32 male. The ages ranged from 52 to 91 years. Twenty-two patients had a smoking history while in 44 it was unknown. There were $40 \mathrm{~T} 1,22 \mathrm{~T} 2,4 \mathrm{~T} 3$ and $0 \mathrm{~T} 4$ tumors. The visceral pleura was positive in 17 cases, negative in 46 and unknown in 3. Forty-nine tumors were grade II, 16 grade III and 1 tumor was grade I. Of 66 patients, 39 had lymph node sampling and 1 was positive. Among the 69 patients with SCC-C, 28 were female and 41 male. The ages ranged from 45 to 80 years. Forty-two patients had a smoking history, 4 were non-smokers and 23 were unknown. There were 45 T2, 15 T1, 9 T3 and no T4 tumors. The visceral pleura was positive in 20 of 69 cases. Thirty-nine tumors were grade I, 28 grade II and 2 grade III. All 69 patients had lymph node sampling and 22 had positive lymph nodes. $M G M T$ promoter methylation was higher in SCC-C than in SCC-P (average $10.9 \%$ vs. $5.3 \%$; $=0.029$ ). Increased methylation levels were seen at all tested cytosines in the $M G M T$ promoter. No differences in promoter methylation were seen in the p16, RASSF1, RASSF5, CDH1, PTEN, SHP1 and LET7 gene promoters.

Hypermethylation of the $M G M T$ promoter at cytosine position -30 was associated with shortened survival in the central group ( 25.3 months vs. median not reached, $\mathrm{p}=0.050$ ) and overall (median 92.0 months vs. median not reached, $\mathrm{p}=0.001$ ).

Conclusions: There was no significant difference in age (mean age 63 years; $\mathrm{p}=0.530$ ) or gender (M:F 41:28 central vs. peripheral 32:34; $\mathrm{p}=0.198$ ). Most SCC-C were grade I and II (67/69), while SCC-P were mostly grade II and III (65/66). SCC-P had a lower pathologic stage T1, 40/66 cases vs. $15 / 69$ cases with SCC-C. In one of 38 patients with SCC-P sampled lymph nodes was positive (T3) vs. 22 with SCC-C had positive lymph nodes. Epigenetic control of $M G M T$ may be a factor in the poor prognosis of SCC-C.

1983 TNFAIP8 Protein Expression in Non Small Cell Lung Cancers Correlates with Histologic Type and Adverse Prognostic Variables

BVS Kallakury, O Voronel, UN Kasid, CE Sheehan, JS Ross. Georgetown University Hospital, Washington, DC; Albany Medical College, Albany, NY.

Background: Tumor necrosis factor alpha induced protein 8 (TNFAIP8) is a transcription factor NF-kB inducible antiapoptotic and oncogenic molecule. This gene was initially discovered by comparison of expression profiles of primary and metastatic head and neck squamous cell carcinoma (hence earlier referred to as SCC-S2). Positive correlation between TNFAIP8 expression and cancer progression/poor prognosis has been described in a few human solid cancers including pancreatic and esophageal primaries. We were the first to publish the adverse prognostic implication of TNFAIP8 expression in prostatic adenocarcinomas. In this study, we evaluated the significance of this protein in non small cell lung cancer (NSCLC).

Design: Formalin-fixed, paraffin-embedded sections from 86 NSCLC, including 22 squamous cell carcinomas (SCC), 31 adenocarcinomas (AC), and 33 bronchioalveolar carcinomas (BAC), were immunostained by a manual method using a custom made antiTNFAIP8 antibody as described earlier (Zhang et al., Int J Cancer, 133: 31-42, 2013). The staining pattern was semiquantitatively assessed based on staining intensity and distribution and the results were correlated with morphologic and prognostic variables. Results: Immunoreactivity for TNFAIP8 was predominantly cytoplasmic. TNFAIP8 protein overexpression was noted in 49/86 $(57 \%)$ tumors and correlated with tumor type [ $81 \%$ AC vs $68 \%$ SCC vs $27 \%$ BAC, $\mathrm{p}<0.0001$ ], overall with advanced tumor stage [ $81 \%$ advanced vs $52 \%$ early, $\mathrm{p}=0.035$ ], lymph node positive status [ $83 \% \mathrm{LN}+\mathrm{vs}$ $51 \% \mathrm{LN}-, \mathrm{p}=0.014]$, and with large tumor size, both overall $[69 \%>3.0 \mathrm{~cm}$ vs $48 \%<=$ $3.0 \mathrm{~cm}, \mathrm{p}=0.05]$ and within the BAC subgroup $[50 \%>3.0 \mathrm{~cm}$ vs $16 \%<=3.0 \mathrm{~cm}, \mathrm{p}=0.05]$. Conclusions: TNFAIP8 immunohistochemical expression portends poor prognosis in NSCLC. Similar to our published data on prostate cancer, this protein may constitute a novel therapeutic target in the management of aggressive NSCLC, warranting further study.

1984 Histological Patterns of Acute Lung Injury in Lung Transplant Patients with Restrictive Allograft Syndrome (RAS)

$S$ Karimi, M Sato, T Saito, HC Roberts, C Chaparro, LG Singer, TK Waddell, S Keshavjee, DM Hwang. University Health Network, Toronto, ON, Canada; Kyoto University, Kyoto, Japan.

Background: Restrictive Allograft Syndrome (RAS) has been recently described as a distinct, aggressive form of chronic lung allograft dysfunction (CLAD) demonstrating restrictive pulmonary function decline and pleuroparenchymal fibroelastosis as a major histopathologic finding. We previously reported that RAS is often also associated with features of prior or concurrent acute lung injury/diffuse alveolar damage. Recently, it has been suggested that acute fibrinoid organizing pneumonia (AFOP) is a new form of CLAD, distinct from RAS. We reviewed specimens from RAS patients to classify histologic patterns of acute lung injury and determine whether histologic features of AFOP are present in patients with RAS

Design: We reviewed surgical pathology cases (transbronchial biopsies, open biopsies, re-transplant pneumonectomies, autopsy lungs) from 16 patients with documented RAS, transplanted at our institution between January 1996 and June 2009. Cases with reported features of acute lung injury were assessed for histologic patterns of acute lung injury by two pathologists with subspecialty pulmonary pathology expertise.

Results: A total of 42 specimens were available for review. Of these, 29 cases demonstrated acute phase changes characterized by alveolar filling by fibrinous exudates (AFOP) and/or classic hyaline membranes (DAD), while the remaining 13 cases showed only organizing phase changes. Of the 29 cases with acute phase changes, 18 cases demonstrated a predominant AFOP (alveolar filling) pattern, while 10 demonstrated predominantly classic DAD (hyaline membrane) pattern, with one case demonstrating approximately equal admixture of both patterns. At least focal features of both patterns were identifiable in the majority of cases (21 of 29).

Conclusions: Histologic features of AFOP are frequently present in lung pathology specimens from patients with RAS, and are commonly present concurrently with features considered more typical of DAD (e.g. hyaline membranes). These findings suggest AFOP to be part of the histologic spectrum of acute lung injury-related changes associated with RAS, rather than a distinct form of CLAD. 


\section{CD200 Is Expressed by Neuroendocrine Carcinoma}

MR Kilgore, SJ Kussick, K Thompson, F Miller, JE Love. University of Washington, Seattle, WA; PhenoPath Laboratories, Seattle, WA; MultiCare, Tacoma, WA; Western Washington Pathology, Tacoma, WA.

Background: CD200 is a membrane bound glycoprotein normally expressed by neurons, endothelial cells, follicular dendritic cells, lymphocytes, macrophages, and granulocytes. CD200 expression may promote tumor formation and metastasis by helping malignant cells evade the immune system. CD200 has been well studied in hematopoietic malignancies including lymphoma, plasma cell myeloma, and acute leukemia. Although less well studied in non-hematopoietic tumors, CD200 expression has been reported in renal cell carcinoma, ovarian carcinoma, neuroblastoma, melanoma, and cutaneous squamous cell and basal cell carcinomas, but not in prostate carcinoma, breast carcinoma, non-small cell lung carcinoma, astrocytoma, or glioblastoma. CD200 expression has not been well-characterized in neuroendocrine neoplasms.

Design: After institutional review, archival, formalin-fixed paraffin-embedded tissues containing small cell carcinoma and Merkel cell carcinoma were identified. Tissue microarrays containing colon carcinoma, prostate carcinoma, breast carcinoma, pulmonary adenocarcinoma, and pulmonary squamous carcinoma were used as negative controls. CD200 expression was evaluated by immuno-histochemistry (IHC) using a goat anti-human CD200 polyclonal antibody (R\&D Systems) at a 1:500 dilution. The immunostains were reviewed by a single pathologist, and scored as follows on the tumor cells: negative $(0 \%$ positive); rare cells positive $(<1 \%)$; focally positive $(1-25 \%)$; variably positive $(25-75 \%)$; and uniformly positive $(>75 \%)$.

Results: CD200 was expressed by 10 of 10 cases $(100 \%)$ of small cell lung carcinoma, and 7 of 7 cases $(100 \%)$ of Merkel cell carcinoma. Tissue microarray studies showed no CD200 expression in 12 of 12 colon carcinoma cores, 15 of 15 breast carcinoma cores, 8 of 8 prostate carcinoma cores, and 52 of 52 pulmonary adenocarcinoma cores. 7 of 9 squamous cell carcinoma cores were negative (78\%), with 2 of $9(22 \%)$ showing focal positivity.

Conclusions: Among the non-hematopoietic neoplasms studied here, CD200 appears to be a highly sensitive marker of small cell lung carcinoma and Merkel cell carcinoma; therefore, CD200 IHC may be helpful in diagnosing these malignancies. It is possible that CD200 expression helps certain tumors evade the immune system, but this area needs to be evaluated in greater detail. Finally, since anti-CD200 antibody therapy is now being developed, CD200 evaluation may have important therapeutic implications for small cell lung carcinoma and Merkel cell carcinoma.

1986 Loss of Syndecan 1 Expression in Tumor and Stromal Cells Is Associated with Higher Stage and Worse Survival in Patients with Lung Adenocarcinoma

M Kohler, JKoblinski, V Parimi, R Raj, K Raparia. Northwestern University, Chicago, IL.

Background: Members of the syndecan family of cell surface heparan sulfate proteoglycans are modulators of growth factor signaling and cell adhesion. Syndecans play a role in a variety of physiological and pathophysiological conditions including migration, angiogenesis, wound healing, tumor growth and progression. The alteration of syndecan 1 expression has been correlated with overall survival in many different tumor types. Clinicopathological implication of Syndecan-1 expression in lung adenocarcinoma has not been well studied.

Design: Immunohistochemical stains were performed on tissue microarray (triplicates with $2 \mathrm{~mm}$ cores) on 162 lung adenocarcinoma samples diagnosed at our institution between 2008 and 2011. 5- $\mu \mathrm{m}$ sections were immunostained with anti-syndecan-1 antibody. The intensity of the membranous and cytoplasmic staining was scored as negative $(0)$, weak $(1+)$, moderate $(2+)$, and strong $(3+)$ in both tumor cells and stroma. Pathologic and clinical characteristics were analyzed and correlated.

Results: Of the 162 patients with lung adenocarcinoma $105(65 \%)$ were females with median age of 67 years (39 to 91 years, SD 10). 18 patients $(11 \%)$ were never smoker and 92 patients $(57 \%)$ were heavy smokers. Syndecan 1 expression in tumor cells was: 0 in $38,1+$ in $68,2+$ in 40 and $3+$ in 8 patients. Syndecan- 1 expression in stromal cells was 0 in $9,1+$ in $55,2+$ in 49 and $3+$ in 41 patients. $63 \%$ patients with loss of syndecan- 1 expression in tumor and stromal cells had higher stage lung disease $(\mathrm{p}=0.024)$. Overall survival was 48 months in patients with no expression of syndecan-1 in tumor and stromal cells as compared to 86 months in patients with expression of syndecan-1 $(\mathrm{p}=0.01)$.

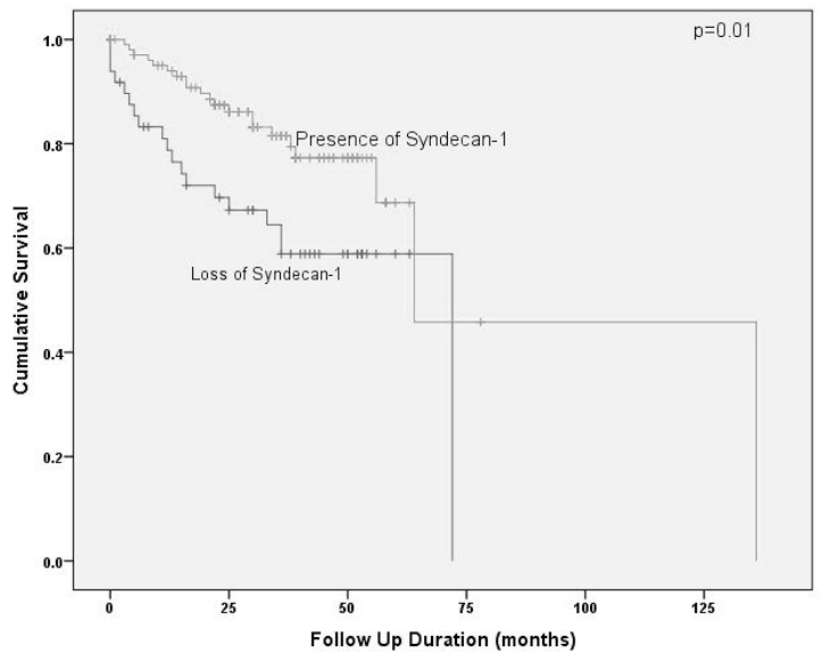

No signifcant association was found beween syndecan-1 expression and presence of brain metastases.

Conclusions: Patients with loss of syndecan-1 expression in tumor and stromal cells in lung adenocarcinomas have higher stage lung disease and worse survival when compared to patients with expression of syndecan-1 in tumor and stromal cells.

1987 Reflex Testing of Small Biopsy Specimens for EGFR and ALK Is Practical and Facilitates Implementation of the 2013 CAP/IASLC/AMP Guidelines for Lung Cancer Biomarker Testing in Patients with Advanced Stage Disease

A Krausert, D Sridhar, H Pass, A Chachoua, J Suh. NYU Langone Medical Center, New York, NY.

Background: The 2013 CAP/IASLC/AMP guidelines for lung cancer biomarker testing recommend EGFR and ALK studies at the time of diagnosis for patients presenting with Stage IV disease or progressive Stage I, II and III disease (P). Since the majority of lung cancer patients are unresectable, the only diagnostic material often consists of small biopsy specimens obtained by interventional radiologists, pulmonologists and surgeons. Our aim is to investigate whether reflex testing of small biopsies is feasible and increases compliance with the new guidelines.

Design: 209 consecutive cases of lung adenocarcinoma, NSCLC-NOS and large cell carcinoma diagnosed by a subspecialty thoracic pathologist at one institution (2011-12) were reviewed. Following a change in departmental policy to reduce reflex testing of surgical resections $(\mathrm{R})$ and begin reflex testing of small biopsies (B), 167 consecutive cases of the same histologies diagnosed by the same pathologist (2012-13) were reviewed. The numbers of total cases, Stage IV/P cases, B and R tested and tested samples that contained sufficient tumor for EGFR and ALK studies were recorded and compared.

Results: From 2011-12, 143/209 (68\%) cases, 36/55 (65\%) Stage IV/P, 38/95 (40\%) B and $105 / 114(92 \%)$ R were tested. $137 / 143(96 \%)$ and $63 / 68(93 \%)$ were adequate for EGFR and ALK, respectively. From 2012-13, 82/167 (49\%) cases, $43 / 48(90 \%)$ Stage IV/P, 73/80 (91\%) B and 9/87 (10\%) R were tested. 79/82 (96\%) and 75/80 (94\%) were adequate for EGFR and ALK, respectively. The differences in the numbers of total cases $(\mathrm{p}$-value $=0.0002)$, Stage IV/P $(\mathrm{p}$-value $=0.0048), \mathrm{B}(\mathrm{p}$-value $<0.0001)$ and $R$ ( $p$-value $<0.0001$ ) tested between the two groups were statistically significant. Of note, the differences in the numbers of tested samples adequate for EGFR and ALK (both p-values $=1.0000$ ) were not statistically significant.

Conclusions: Reflex testing of small biopsy specimens for EGFR and ALK is practical and facilitates implementation of the new lung cancer biomarker testing guidelines, which are intended to enable oncologists and patients with advanced stage disease to choose optimal therapies. Furthermore, B are usually collected in the outpatient setting, where molecular testing is reimbursed without a waiting period.

1988 Chromosomal Abnormalities between Atypical Adenomatous Hyperplasia, Adenocarcinoma In Situ and Invasive Adenocarcinoma by FISH

TN Kroneman, JS Voss, MB Campion, KC Halling, J Zhang. Mayo Clinic College of Medicine, Rochester, MN.

Background: Diagnosis of atypical adenomatous hyperplasia (AAH), adenocarcinoma in situ (AIS) and invasive adenocarcinoma (IA) may be challenging and arbitrary during histologic examination of lung resection specimens. The goal of this pilot study was to determine if there are differences in chromosomal abnormalities between these lesions using fluorescence in situ hybridization (FISH).

Design: Paraffin-embedded specimens containing AAH, AIS or IA were reviewed by two pathologists and hybridized with FISH probes to $7 \mathrm{p} 12$ (EGFR), 5p15, CEP 6, and $8 \mathrm{q} 24$ (MYC). A cytotechnologist enumerated signal patterns in up to 100 tumor cells from areas identified as AIS, AAH, or IA and up to 50 cells from 29 normal regions. Polysomy was defined as $\geq 3$ signals for $\geq 2$ probes and a single locus gain was defined as $\geq 3$ signals for one probe. A specimen was considered FISH abnormal when $3 \%$ of cells exhibited polysomy or $10 \%$ of cells exhibited single probe gain. 
Results: Seventy areas were enumerated from the 26 patients (mean age 67.7, range 45-87) included in the study (10 male and 16 female). Primary lung cancers were diagnosed in 15,5 had metastatic cancers to the lung and 6 had a benign diagnosis. Results are summarized in Table 1 .

\begin{tabular}{|c|c|c|c|c|}
\hline Histology & Areas counted & $\begin{array}{l}\begin{array}{l}\text { Single probe gain }(10 \% \\
\text { cutoff })\end{array} \\
\text { ? }\end{array}$ & $\begin{array}{l}\text { Polysomy (3\% } \\
\text { cutoff) }\end{array}$ & combined \\
\hline Normal & 29 & $0 / 29(0 \%)$ & $0 / 29(0 \%)$ & $0 / 29(0 \%)$ \\
\hline AAH & 23 & $12 / 23(52 \%)$ & $15 / 23(65 \%)$ & $18 / 23(78 \%)$ \\
\hline AIS & 11 & $7 / 11(64 \%)$ & $11 / 11(100 \%)$ & $11 / 11(100 \%)$ \\
\hline IA & 7 & $4 / 7(57 \%)$ & $77 / 7(100 \%)$ & $7 / 7(100 \%)$ \\
\hline
\end{tabular}

There was a marked difference $(\mathrm{P}<0.001)$ in the frequency of cases with single probe gain and polysomy when comparing normal tissue to AAH, AIS and IA. And furthermore, there was a significant difference $(P<0.006)$ in the frequency of cases with polysomy when comparing AAH to AIS and IA. Single probe gain was found in $52 \%, 64 \%$ and $57 \%$ of AAH, AIS and IA, respectively. Polysomy was detected in all cases with AIS or IA and $65 \%$ of AAH. When single probe gain and polysomy were combined, three additional cases $(78 \%)$ of AAH were considered FISH abnormal.

Conclusions: These data suggest there is a significant difference in the frequency of cases with polysomy or single probe gain between normal histology, AAH and AIS/ IA. And these findings indicate chromosomal abnormalities detected by FISH can be used as an ancillary test aiding in the diagnosis of challenging cases between normal/ AAH and AIS/IA on surgical resection specimens and potentially on challenging cytologic specimens.

1989 Diagnostic Utility of Scanning Electron Microscopic Measurement of Microvilli in Epithelioid Malignant Mesotheliomas Using ParaffinEmbedded Biopsy Material

KY Kwon, SH Jung, YD Choi, HS Park, SY Ha, SH Lee, JH Han, SH Chang, JA Ghafar, WI Choi, HR Jung, I Hwang. Keimyung University Dongsan Medical Center, Daegu, Korea; Yonsei University Wonju College of Medicine, Wonju, Korea; The Cardiopulmonary Pathology Study Group of Korean Society of Pathologists, Seoul, Korea.

Background: Recent application of immunohistochemistry (IHC) using new developed markers for the diagnosis of malignant mesothelioma (MM), along with the short turnaround time (TAT) for results, has made the diagnostic role of electron microscopy (EM) very restricted. However, some cases of malignant mesothelioma demonstrate indefinite results for IHC stains and need supplemental tools for an accurate diagnosis of MM. Scanning EM (SEM) using paraffin-embedded biopsy samples is therefore a simple and useful tool for accurately diagnosing cases of MM that are indistinct from metastatic adenocarcinoma (ADC) using IHC

Design: A total of 30 cases involving the pleura or peritoneum were examined: 20 cases of epithelioid MM (EMM) and 10 cases of metastatic ADC. The length and diameter of 20 microvilli in the intercellular spaces of each case was measured from paraffinembedded tissue on unstained slides after modified SEM procedure. The TAT for SEM procedure using paraffin-embedded tissue is about 4 hours. The Mann-Whitney $U$ test was used for comparison of the mean length, diameter, and length/diameter ratio. Receiver operating characteristic curve analysis was used to identify the appropriate length/diameter ratio cutoff value and the Chi-square test was used to determine sensitivity and specificity

Results: EMM tumor cells generally displayed very long and thin apical microvilli. In contrast, ADC tumor cells exhibited short microvilli with slightly broad bases. The mean length and diameter of EMM microvilli were $2.42( \pm 0.86) \mu \mathrm{m}$ and $0.34( \pm 0.32)$ $\mu \mathrm{m}$, whereas those for ADC microvilli were $0.85( \pm 0.39) \mu \mathrm{m}$ and $0.32( \pm 0.09) \mu \mathrm{m}$, respectively. The mean length/diameter ratio for EMM was $9.50( \pm 2.58)$ and that for $\mathrm{ADC}$ was $2.71( \pm 0.85 ; p<0.001)$. The diagnostic length/diameter ratio cutoff value between EMM and ADC was $4.2(p<0.001)$, and sensitivity and specificity were $96 \%$ and $100 \%$, respectively.

Conclusions: The SEM procedure using paraffin-embedded biopsy material for analysis can be applicable to the accurate diagnosis of EMM and can be a supplementary tool for the differential diagnosis between EMM and metastatic ADC.

\section{Clinicopathologic Features of Non-Small Cell Lung Cancer (NSCLC) with ALK Gene Rearrangement Based on New (2011) IASLC/ATS Classification and FDA-Approved ALK Assay}

NS Lakis, S Zhang, J Tull, M Stram. SUNY Upstate Medical University, Syracuse, NY. Background: Anaplastic lymphoma kinase (ALK) gene rearrangement is a predictive biomarker of treatment response to ALK kinase inhibitor and has been used to select patients with NSCLC for ALK-targeted therapy. ALK rearrangement accounts for 2-5\% of NSCLC. The clinicopathologic features of ALK rearrangement are not well studied, especially based on the FDA-approved ALK assay and the new (2011) classification of lung adenocarcinoma by the International Association for the Study of Lung Cancer (IASLC)/American Thoracic Society (ATS) criteria.

Design: Five hundred and twenty-one cases of primary or metastatic NSCLC diagnosed over 22 months in multiple central New York hospitals were tested for ALK rearrangement using the FDA-approved Vysis ALK Break Apart FISH Probe Kit (Abbott Molecular Inc.) and evaluated according to the manufacturer's guidelines. All cases were blinded and classified based on the 2011 criteria for lung adenocarcinoma as lepidic, acinar, papillary, micropapillary or solid predominant patterns, mucinous carcinomas and any signet ring/intracellular mucin component. The patients' age, sex and smoking status (if ALK+) were recorded.

Results: In line with previously published data, $2.2 \%$ of the cases were ALK $+(\mathrm{n}=11)$. ALK+ patients' average age was 51 with a male to female (M:F) ratio of 1.2:1.0. ALK- patients' average age was 67 with the same M:F ratio of 1.2:1.0. The smoking status of 6 ALK+ cases was known: 4 current/former smokers and 2 non-smokers. Table 1 shows the comparative breakdown of the predominant histologic patterns of the ALK+ and ALK- cases.

\begin{tabular}{|c|c|c|c|c|c|c|c|c|c|c|c|}
\hline \multicolumn{12}{|c|}{ Table 1} \\
\hline & $\begin{array}{l}\text { Total } \\
\text { No. }\end{array}$ & $\begin{array}{l}\text { Ave. Age } \\
\text { (range) }\end{array}$ & M:F & Lepidic & Acinar & Papillary & $\begin{array}{c}\text { Miver- } \\
\text { papillary }\end{array}$ & $\begin{array}{c}\text { solld } \\
\text { with } \\
\text { silos } \\
\text { signet } \\
\text { ring } \\
\text { cellils }\end{array}$ & $\begin{array}{l}\text { Solld } \\
\text { with } \\
\text { witox } \\
\text { signet } \\
\text { ring } \\
\text { cellis }\end{array}$ & Mucinous & $\begin{array}{c}\text { Not } \\
\text { classifiable }\end{array}$ \\
\hline ALK+ & 11 & $\begin{array}{c}51 \\
(27-69)\end{array}$ & 1.2 & 0 & $\begin{array}{c}2 \\
(186)\end{array}$ & 0 & 0 & $\begin{array}{c}3 \\
(278)\end{array}$ & \begin{tabular}{c|}
4 \\
$(36 \times)$
\end{tabular} & $\begin{array}{l}1 \\
(96)\end{array}$ & $\begin{array}{l}1 \\
(9 \times)\end{array}$ \\
\hline ALK. & 510 & $\begin{array}{l}67 \\
63901\end{array}$ & 1.2 & $\frac{24}{2450}$ & $\begin{array}{l}134 \\
12640\end{array}$ & $\begin{array}{l}33 \\
65654\end{array}$ & 5 & $\begin{array}{l}155 \\
13094\end{array}$ & \begin{tabular}{|l|l|}
17 \\
354
\end{tabular} & $\begin{array}{l}13 \\
125 \%\end{array}$ & $\begin{array}{l}129 \\
125 \% 1\end{array}$ \\
\hline
\end{tabular}

Conclusions: ALK+ carcinomas were found in patients who were, on average, a decade younger than those with ALK- carcinoma. Contrary to previous reports, $2 / 3$ of $\mathrm{ALK}+$ patients with complete histories were smokers $(\mathrm{n}=6) . \mathrm{ALK}+$ carcinomas had a significantly higher proportion of solid predominant pattern with $\geq 10 \%$ signet ring cells than the ALK- ones $(36 \%$ vs $3 \%, p<0.001)$. Carcinomas with lepidic, papillary, or micropapillary predominant patterns were unlikely to be ALK + . Identifying clinically relevant patterns allows for more accurate identification of patients who may benefit from ALK rearrangement testing and targeted therapy.

1991 Epigenetic Regulation of BAX Gene in Neuroendocrine Tumors of the Lung and Its Prognostic Implication

I Lamzabi, LE Rosen, L Buckingham, $R$ Jain, $W$ Barbanera, P Bitterman, $V$ Reddy, $P$ Gattuso. Rush University Medical Center, Chicago, IL.

Background: BAX is a member of the $\mathrm{Bcl}-2$ gene family that promotes apoptosis by binding to and antagonizing the $\mathrm{Bcl}-2$ protein. It has been well studied in lung tumors with the exception of neuroendocrine lung tumors (NELT); and to our knowledge, there is no published data regarding epigenetic regulation of BAX in this group of neoplasms. The following study was undertaken to investigate the role of epigenetics in the expression of BAX protein.

Design: Formalin fixed paraffin embedded tissues from 41 cases of primary NELT including 14 small cell carcinomas (SCLC), 18 typical carcinoids (TC) and 9 atypical carcinoids (AC) were included in the study. Tissue sections were immunostained with BAX antibody to determine the expression. DNA extracted from microdissected tumor tissue was treated with sodium bisulfite and amplified by PCR using primers to the BAX gene promoter, followed by pyrosequencing. Data was analyzed by Pearson correlation using SPSS statistical software.

Results: Loss of BAX expression was positively associated with distant metastases $(p<0.001)$ in NELT and SCLC group $(p<0.005)$. Hypermethylation at cytosines -79 and -75 of the BAX promoter (A of ATG $=+1$ ) correlated with distant metastases ( $\mathrm{p}=0.018$ and $p=0.019$ respectively), while hypermethylation at cytosines -57 and -50 correlated with lymph node metastasis ( $\mathrm{p}=0.016$ and $\mathrm{p}=0.009$ respectively). In addition, there was more methylation of the -79 (average 8.42 vs $3.8(\mathrm{p}=0.02)$ ) and -75 (average 9.28 vs $3.8(\mathrm{p}=0.01)$ ) sites in SCLC than TC. Lymph node metastasis was associated with cytosine -79 hypermethylation in SCLC $(\mathrm{p}=0.01)$ and -57 and -50 hypermethylation in $\mathrm{TC}(\mathrm{p}=0.01$ and $\mathrm{p}=0.009$ respectively). However, only weak correlation between BAX expression and BAX promoter methylation was observed.

Conclusions: BAX protein appears to be associated with tumor behavior as its loss predict distant metastases in NELT overall, and SCLC specifically. This loss of BAX is likely due to an epigenetic phenomenon through a preferential methylation at -79 and -75 sites of the BAX gene promoter. In addition, lymph node metastases in TC were associated with hypermethylation of -57 and -50 sites. The lack of a strong direct correlation between methylation and expression might be due to the interference of post-translational events or additional factors that are yet to be elucidated.

1992 Bcl-2-Associated X Protein (BAX) Expression in Neuroendocrine Lung Tumors

I Lamzabi, LE Rosen, L Buckingham, R Jain, W Barbanera, P Gattuso. Rush University Medical Center, Chicago, IL.

Background: $\mathrm{BAX}$ is a member of the $\mathrm{Bcl}-2$ gene family. Apoptosis regulator BAX promotes apoptosis by binding to and antagonizing the $\mathrm{Bcl}-2$ protein. It has been well studied in lung tumors with the exception of neuroendocrine carcinomas of the lung (NELT), including typical (TC) and atypical carcinoids (AC). In this study, we investigate the expression of BAX protein in small cell lung carcinomas (SCLC), TC and $\mathrm{AC}$ of the lung; and its relationship to tumor characteristics and behavior

Design: We searched our surgical pathology records from 1993 to 2011 for cases of typical and atypical carcinoid of the lung with available tissue. Cases of SCLC were randomly selected from an institutional database. Pertinent clinicopathological data was also collected. $\mathrm{H} \& \mathrm{E}$ slides were reviewed and the diagnoses were confirmed. The cases were then immunostained with BAX antibody, and the immunostain was interpreted by two different pathologists. Data was analyzed by Pearson Correlation on SPSS software. Results: Sixty seven cases of neuroendocrine lung tumors were studied ( $10 \mathrm{AC}, 30$ $\mathrm{TC}$, and $27 \mathrm{SCLC}$ ). Lymph node metastases were seen in $3 \mathrm{AC}, 4 \mathrm{TC}$, and 7 SCLC. Distant metastases were present in $1 \mathrm{AC}, 1 \mathrm{TC}$, and 18 SCLC. When cases were combined, higher expression of BAX was seen in younger patients $(p<0.001)$ and loss of BAX was positively associated with distant metastases $(p<0.001)$. SCLC showed a significant loss of $\mathrm{BAX}$ compared to carcinoid tumors, $\mathrm{TC}$ and $\mathrm{AC}(\mathrm{p}=0.005$ and $\mathrm{p}=0.003$ respectively). Additionally, in the SCLC group, loss of BAX expression was positively associated with distant metastases $(\mathrm{p}=0.03)$. Higher expression of BAX correlated with tumor size in only the $\mathrm{AC}$ group $(\mathrm{p}=0.02)$. There was no difference in $\mathrm{BAX}$ expression between the TC and AC groups and no association with distant or lymph node metastases in either TC or AC. 
Conclusions: Loss of BAX expression correlates with grade in NELT, as SCLC showed loss of BAX expression when compared to TC and AC. Loss of BAX expression is also positively correlated with distant metastases in SCLC. Therefore, BAX appears to play an important role in the metastatic behavior of SCLC. BAX does not appear to interfere with or regulate the biologic behavior of TC and AC.

1993 Distribution of Interstitial Lung Diseases in the Elderly: A Clinicopathologic Analysis of 107 Patients at Least 75 Years Old Requiring Surgical Lung Biopsy (2000-2012)

BT Larsen, JH Ryu, LT Vaszar, HD Tazelaar. Mayo Clinic, Rochester, MN; Mayo Clinic, Scottsdale, AZ

Background: Idiopathic pulmonary fibrosis (IPF) is the most common interstitial lung disease (ILD) in elderly patients, and is increasingly diagnosed by imaging alone if definite radiologic features of usual interstitial pneumonia (UIP) are seen. Other elderly patients with ILDs without diagnostic imaging findings may undergo a surgical lung biopsy, but the distribution of ILDs in this vulnerable group has not been characterized. Design: All wedge biopsies from patients at least 75 years old with ILD (2000-2012) were identified by searching databases of Mayo Clinic in Minnesota and Arizona. Slides were retrieved and histologic patterns were reviewed.

Results: Among 107 patients (mean age 79 yrs, range 75-87; 69 men), 43 presented with acute respiratory failure and 64 presented in the ambulatory setting. Biopsies included at least 2 separate lobes/sites in 82 cases (77\%). Of acutely ill patients, only half $(21 ; 49 \%)$ showed fibrosis, almost always UIP (20), often (15) with superimposed diffuse alveolar damage (DAD), acute lung injury (ALI), or organizing pneumonia (OP), consistent with acute exacerbation of IPF or other fibrosing ILD. Those without fibrosis showed a variety of findings, including ALI (8), DAD (7), small airway disease (5), granulomas (4), OP (3), eosinophilic pneumonia (3), and/or foamy macrophages (3), suggestive of infections, drug reactions, and other disorders. One showed alveolar hemorrhage. Of ambulatory patients, most $(48 ; 75 \%)$ showed UIP-like fibrosis, but more than half of these cases (27) also showed findings suggesting alternate diagnoses such as chronic hypersensitivity pneumonitis or connective tissue disease, most commonly granulomas (15) or lymphoid hyperplasia (7). The other ambulatory cases showed a variety of findings including granulomas (7), OP (3), small airway disease (3), eosinophilic pneumonia (2), unclassifiable fibrosis (2), amyloidosis (1), and ErdheimChester disease (1). Taken together, only 42 (39\%) of acutely ill and ambulatory patients showed histologic features compatible with IPF.

Conclusions: In elderly patients with ILD who require biopsies for diagnosis, UIP remains the most common histologic pattern, but more than half of biopsies from both acutely ill and ambulatory patients show evidence of alternate diagnoses incompatible with IPF. Even in elderly patients, surgical lung biopsy often provides valuable information to guide clinical management

1994 Analysis of Genetic Alteration in Lung Squamous Cell Carcinoma Using Multiplex Ligation-Dependent Probe Amplification

B Lee, MJ Kown, J-Y Song, Y-L Choi. Samsung Medical Center, Sungkyunkwan University School of Medicine, Seoul, Republic of Korea; Hallym University Sacred Heart Hospital, Hallym University, College of Medicine, Anyang, Republic of Korea; Samsung Biomedical Research Institute, Samsung Medical Center, Seoul, Republic of Korea; Institute for Refractory Cancer Research, Samsung Medical Center, Seoul, Republic of Korea.

Background: Most targeted therapy in non-small cell lung cancer has been focused on adenocarcinoma. Recently FGFRl amplification is found to be present in up to $20 \%$ of lung squamous cell carcinoma (SqCC). Several FGFR1 tyrosine kinase inhibitors are currently undergoing clinical trial. Other potential therapeutic targets of lung $\mathrm{SqCC}$ are EGFR, KRAS, PIK3CA, BRAF, ERBBS, and DDR2. We evaluated FGFRI amplification and several other genetic alterations in lung SqCC cases from Korean patients.

Design: 291 cases of SqCC were obtained from pathology files. FGFRl copy number was detected using fluorescence in situ hybridization (FISH). Clinical significance of FGFRI amplification was measured using Kaplan-Meier survival analysis. For detection of multiple genetic alterations, we used the MLPA SALSA X050-A1 Lung cancer kit (MRC-Holland, Amsterdam). This probe mix contains 27 probes for detecting copy number of PIK3CA, EGFR, MET, FGFR1, PREN, KRAS and ERBB2. It also contains 9 specific probes for the mutations of PIK3CA, EGFR, BRAF, KRAS and ERBB2.

Results: We defined $F G F R 1$ amplification when $F G F R 1$ copy number was greater than 9. Disease-free survival and overall survival was not significantly different between $F G F R 1$ amplification group and non-amplification group. MLPA results showed amplification of FGFRI (12.7\%), PIK3CA (4.8\%), EGFR (4.5\%), ERBB2 (7.2\%). $P I K 3 C A$ mutations were detected in $2.4 \%$ of tumor specimens. Compare to FISH results of $F G F R 1$, the sensitivity of MLPA was $63 \%$.

Conclusions: FGFR1 amplification status was statistically not related to survival in most previously published reports. Our data showed no survival difference between FGFR1 amplification and non-amplification group. Using MLPA, 167 cases(57\%) were found to have one or more mutations. Most of the mutations have targeting drugs. This method can be useful for finding suitable drugs for advanced lung SqCC patients.
1995 Two New ALK Novel Fusion Proteins Identified in Lung

\section{Adenocarcinoma}

B Lee, M Hong, M Lira, M Mao, K Takeuchi, JHan, J Kim, Y-L Choi. Samsung Medical Center, Sungkyunkwan University College of Medicine, Seoul, Republic of Korea; Pfizer Oncology, San Diego, CA; Pathology Project for Molecular Targets of The Cancer Institute/Division of Pathology of the Cancer Institute Hospital, Japanese Foundation for Cancer Research, Tokyo, Japan; Samsung Medical Center, Sungkyunkwan University College of Medicine, Seoul, Korea; Institute for Refractory Cancer Research, Samsung Medical Center, Seoul, Korea.

Background: The most common mechanism for overexpression and activation of anaplastic lymphoma kinase (ALK) in non-small cell lung carcinoma (NSCLC) could be attributed to the formation of a fusion protein. To date, five fusion partners of ALK have been reported, namely, EML4, TFG, KIF5B, KCL1 and PTPN3.

Design: Here, we report two novel fusion genes $H I P 1-A L K$ and $T P R-A L K$. Reverse transcriptase polymerase chain reaction and immunohistochemical analysis were used to detect this fusion gene's transcript and protein expression, respectively. We had amplified the full-length cDNA sequence of this novel fusion gene using 5 '-rapid amplification of cDNA ends (5'RACE).

Results: The causative genomic translocation $\mathrm{t}(2 ; 7)(\mathrm{p} 23 ; \mathrm{q} 11.23)$ and $\mathrm{t}(1 ; 2)(\mathrm{q} 31.1 ; \mathrm{p} 23))$ for generating this novel fusion gene was verified using genomic sequencing. In breakapart fluorescence in situ hybridization analysis for $A L K$, split of the 5' and 3' probe signals were observed. Reverse transcriptase polymerase chain reaction revealed that the tumor harbored a novel fusion transcript in which exon 21 of $H I P 1$ was fused to exon 20 of $A L K$ in-frame.

Conclusions: The novel fusion genes and their proteins HIP1-ALK and TPR-ALK harboring Coiled-coil and kinase domains, which could play a role in carcinogenesis, could become diagnostic and therapeutic target of the NSCLCs and deserve a further study in the future.

1996 The Differential Role of Sp1 and HIF-1 $\alpha$ in the VEGF Induction in Non Small Cell Lung Carcinoma and Their Clinical Significance

HJ Lee, KB Kim, DH Shin, JH Lee. School of Medicine, Yang San, Kyung Sang Nam Do, Republic of Korea.

Background: Vascular endothelial growth factor (VEGF) is one of the most important molecule inducing tumor angiogenesis and its upregulation is related with poor prognosis in various cancers. HIF- $1 \alpha$ has been thought to be important activator of VEGF expression. However, studies regarding the relationship between HIF-1 $\alpha$ and VEGF in non-small cell lung carcinoma (NSCLC) showed variable results. Recently, several studies indicated transcriptional factor $\mathrm{Sp} 1$ is involved in cancer related angiogenesis. Hence, we thought the discrepancy between HIF-1 $\alpha$ and VEGF in NSCLC may be explained by $\mathrm{Sp} 1$

Design: AA549 human lung adenocarcinoma cell line was transfected with anti-Sp1 siRNA. The mRNA level was compared using quantitative real time PCR and protein expression was shown by immunocytochemistry and western blot. TMA blocks from 66 squamous cell carcinoma (SqCC) and 80 adenocarcinoma were prepared to perform immunohistochemistgry for Sp1, HIF-1 $\alpha$ and VEGF. The ordinal regression test was used to evaluate the statistical association between Sp 1, HIF-1 $\alpha$, and VEGF expression in NSCLC. The Pearson chi-square test was used to evaluate the statistical significance of immunohistochemical results related to clinicopathological parameters. Follow-up information was also obtained for survival analysis.

Results: After anti-Sp1 siRNA transfection, mRNA levels of Sp1 and VEGF were decreased simultaneously. On immunocytochemistry, Sp1 and VEGF expressions were reduced significantly, implying $\mathrm{Sp} 1$ regulates VEGF expression. Immunohistochemistry showed a significant relationship of HIF-1 $\alpha$ and VEGF expression in SqCC $(p=0.02)$ but not in adenocarcinoma ( $p=0.78)$. Sp1 was associated with VEGF expression in both of histology ( $p=0.03$ for SqCC and $p=0.005$ for adenocarcinoma). Sp1 expression was also related with higher T stage $(p=0.04)$ and PCNA index $(p=0.04)$ and showed a near significant correlation with overall survival $(p=0.08)$.

Conclusions: we suggest that two different transcription factors are involved in the induction of VEGF expression in SqCC and adenocarcinoma of NSCLC. Sp1 plays an important role in induction of VEGF expression in both histologic types of NSCLC, but this role is more dominant in adenocarcinoma. Sp1 seems to be involved not only in angiogenesis, but also in cell proliferation and growth contributing to tumor progression. However, regarding the role of $\mathrm{Sp} 1$ as a prognostic marker, further investigation is needed.

1997 KRAS Mutation Profiling of Non-Small Cell Lung Cancer by Using PNA-Mediated PCR Clamping Method

$B$ Lee, B Lee, Y-L Choi. Samsung Medical Center, Sungkyunkwan University School of Medicine, Seoul, Republic of Korea.

Background: The two most commonly mutated genetic "driver" in some non-small cell lung cancers (NSCLCs) encode for the epidermal growth factor receptor (EGFR) and KRAS. Recent studies indicate that patients with mutant KRAS tumors fail to benefit from adjuvant chemotherapy, and their disease does not respond to EGFR inhibitors. Furthermore, targeting of KRAS mutant NSCLCs has been tried recently. For those, the feasible mutation detection method is required for the accurate test of $K R A S$ status. In this study, we compare two methods in detecting KRAS mutations of NSCLCs, exploring associations with clinicopathologic factors.

Design: 136 cases of primary NSCLC was obtained from pathology files spanning 2012 to 2013. Clinical information including stage and smoking history was obtained based on clinical records. Direct sequencing and PNA-mediated PCR clamping was performed to all cases. Next generation sequencing (NGS) was used to validate 
discordant cases. To increase resolution of low level somatic mutant molecules within a high background of wild-type molecules, PNA-mediated PCR clamping was used for mutant enrichment before NGS.

Results: $21(15.7 \%)$ of the 136 cases were found to have missense mutation by direct sequencing. When using PNA-mediated PCR clamping method two more cases (case 92 and case 133) were found to have missense mutation. Case 92 is a sample from mucinous carcinoma. The size of tumor was $1 \mathrm{~cm}$ and the tumor volume contained in the sample was about $20 \%$. Case 133 is from adenocarcinoma resected after neoadjuvant chemotherapy, showing only about $5 \%$ of the tumor volume in specimen. The percentage of $K R A S$ mutation detected in case 92 and case 133 is $2 \%$ and $4 \%$, respectively in NGS. $K R A S$ mutation allele was definitely increased in NGS after using PNA-clamping up to $90 \%$ and $89 \%$ in both cases. Clinically, KRAS mutation tend to be present in tumors of smoker $(\mathrm{p}=0.012)$ and stage IV tumors had more frequent $K R A S$ mutation $(\mathrm{p}=0.032)$. Conclusions: Although statistical significance was not reached, more cases were found to have KRAS mutation when PNA-mediated PCR clamping method was used. The result of two discordant case was confirmed by NGS method. Direct sequencing can accurately detect mutations when percentage of tumor cells in the analytical sample is enough. However, we cannot always get enough tumor volume. In that situation, PNAmediated PCR clamping method can quickly provide result with sufficient sensitivity.

1998 RNA In Situ Hybridization Is a More Sensitive Method Than Immunohistochemistry in Detection of Napsin A Expression in Lung Adenocarcinomas

F Lin, J Shi, H Liu. Geisinger Medical Center, Danville, PA.

Background: Expression of napsin A and thyroid transcription factor 1 (TTF1) by immunohistochemistry (IHC) was reported in approximately $75 \%$ of primary lung adenocarcinomas (ADCs), and this pair of immunomarkers plays a crucial role in differentiating lung ADC from lung squamous cell carcinoma (SCC) and identifying a primary lung ADC when working on a tumor of unknown origin. RNA in situ hybridization (RNAscope) is a newly described, highly sensitive technique using a bright-field in situ hybridization-based assay to visualize targeted cellular RNA in formalin-fixed paraffin-embedded tissue sections. Because approximately $25 \%$ of primary lung ADCs are negative for both napsin A and TTF1 by IHC, there is a need to explore a more sensitive technique to detect these biomarkers. In this study we investigated the diagnostic sensitivity of RNAscope in the detection of napsin A expression in primary lung ADCs and compared it to IHC techniques.

Design: IHC for napsin A (clone EP205) was performed on tissue microarray (TMA) sections containing 76 lung ADCs and 80 lung SCCs. RNAscope was also performed on these TMA sections to detect mRNA of napsin A according to the protocol from Advanced Cell Diagnostics, Inc. The procedure included de-paraffinization, pretreatment, target probe hybridization, signal amplification, and colorimetric reaction. The targeted probes prepared by the same vendor covered napsin A sequence from nucleotides 233 to 1172 . The scoring system for RNAscope was based on these criteria: 0 ( $1 \mathrm{dot} /$ tumor cell $), 1+(2-3$ dots/tumor cell $), 2+(4-10 \mathrm{dots} /$ cell $), 3+(>10$ dots/cell), and $4+(>10$ dots/cell and more than 10 cells $)$. The IHC staining intensity and distribution were recorded.

Results: The result of RNAscope for napsin A showed that $89.5 \%$ lung ADCs had detectable mRNA, with $4+$ in $74 \%$ cases, whereas only $76 \%$ were positive by IHC. The RNAscope signal was easily detectable even at low magnification. An example of lung ADC with 4+ positivity by RNAscope is shown in the figures below. Napsin A expression was not seen in SCCs using either IHC or RNAscope.

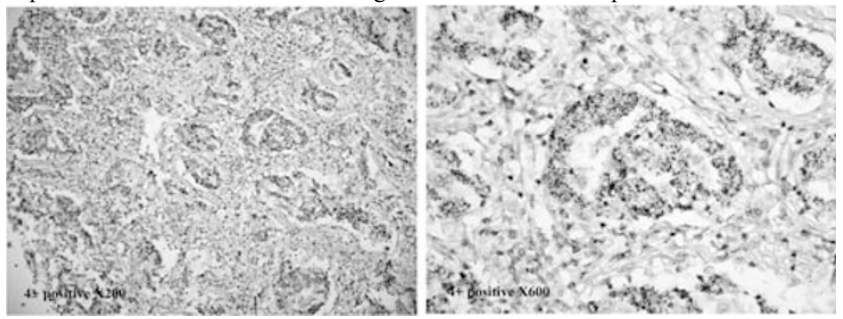

Conclusions: These data suggest that RNA in situ hybridization technique is superior to IHC in detecting napsin A expression in primary lung adenocarcinomas.

1999 Histologic Features and Ki-67 Proliferative Index of Excised Neuroendocrine Tumors of the Lung

SZ Liu, BA Alexiev, KC Tuttle, AP Burke. University of Maryland, Baltimore, MD Background: The histopathologic distinction between carcinoids, atypical carcinoids and large cell neuroendocrine tumors is based partly on mitotic index. Mitotic counts can be difficult to assess, especially on small biopsies or when the rate is low. To date, there are no established criteria for interpreting quantitative Ki-67 index for pulmonary neuroendocrine tumors.

Design: We identified 52 lung neuroendocrine tumors and classified them into typical carcinoid ( $\mathrm{n}=35)$, atypical carcinoid $(\mathrm{n}=7)$, and high-grade neuroendocrine carcinomas (8 large cell/LCNEC, 1 recurrent small cell carcinoma). Two LCNEC had areas of adenocarcinoma, one in situ. Ki-67 proliferative index was calculated using validated nuclear algorithm on Aperio digital image analysis on representative tumor.

Results: There was a strong correlation between mitotic count and Ki-67 index $\left(\mathrm{R}^{2}=0.81, \mathrm{p}<0.001\right)$. The mean mitotic count and Ki-67 for typical, atypical, large cell neuroendocrine tumors were $0.05 / 2.52 \%, 4.14 / 16.84 \%$, and $48.44 / 71.59 \%$ respectively.

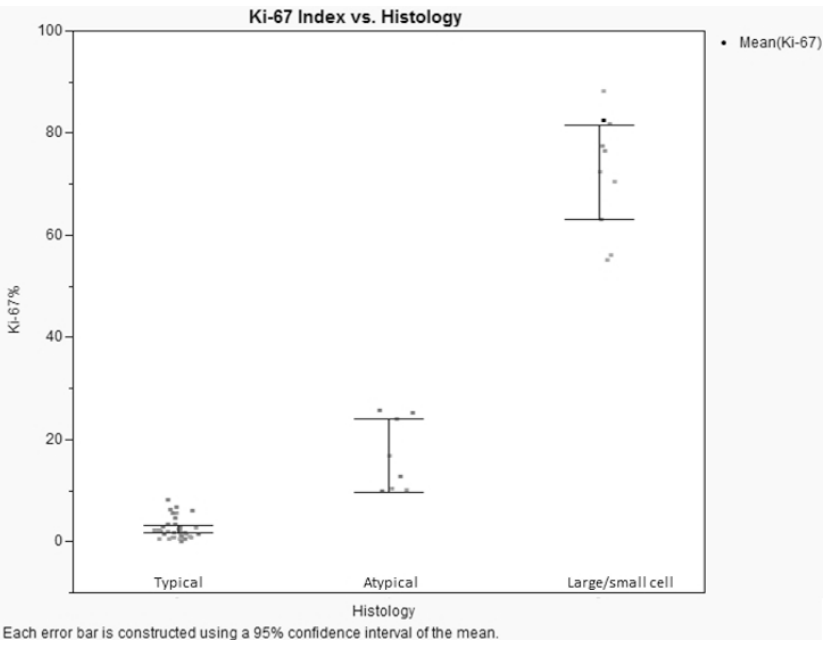

There were significant differences in mitotic index and Ki-67 between typical, atypical, and large cell/small cell neuroendocrine tumors $(\mathrm{p}<0.001)$. For typical and atypical neuroendocrine tumors, there was no correlation between mitotic count or Ki-67 and tumor size, tumor stage, or nodal stage. Two typical carcinoid tumor had nodal metastasis while one atypical carcinoid had a nodal metastasis. Both typical and atypical carcinoid tumors did not reoccur after resection. Seven typical carcinoids and two atypical carcinoids had spindled morphology. LCNECs showed areas of insular (6/8), trabecular $(4 / 8)$, and focally glandular (2/8) and spindled growth (1/8); in some areas the tumor was indistinct from small cell carcinoma. Two LCNEC had areas of adenocarcinoma, one in situ. Tumor necrosis was universally present in LCNEC tumors but only present in $2 / 7$ atypical carcinoid tumors.

Conclusions: Ki-67 index separates typical from atypical carcinoids at a level of $10 \%$ quantitative labeling. Large cell neuroendocrine carcinomas all have a Ki-67 index of $>50 \%$, similar to small cell carcinomas, and, at resection, often have areas resembling small cell carcinoma.

2000 Correlation of Twist Expression in Different Histologic Subtypes of Primary Adenocarcinoma in Lung

A Malek, C O'Hara, H Kathuria. Boston Medical Center, Boston, MA

Background: Tumor recurrence and metastasis have significant impact on the mortality of lung cancer. Metastasis is particularly associated with early demise. Transcription factors that mediate the epithelial-mesenchymal transition(EMT) play a major role in facilitating metastasis by a variety of mechanisms. Twist a basic helix-loop-helix transcription factor is well known to be involved in promoting metastasis. The role of Twist in lung cancer has not been studied well due to the lack of a reproducible classification. This has been addressed by the International Association for the Study of Lung Cancer which has published guidelines for a multidisciplinary classification of lung adenocarcinomas that highlights the heterogeneity of lung adenocarcinomas and establishes a uniform classification. In the new system, lung adenocarcinomas are classified as lepidic, acinar, papillary, micropapillary and solid types; each with prognostic significance. This study attempts to correlate Twist positivity with the various subtypes and account for the prognostic variability encountered in the treatment and outcome of patients.

Design: A total of 30 lung adenocarcinomas were subtyped according to the IASLC proposal and stained with Twist monoclonal antibody. Twist staining less than $10 \%$ was considered negative, greater than $10 \%$ considered positive.

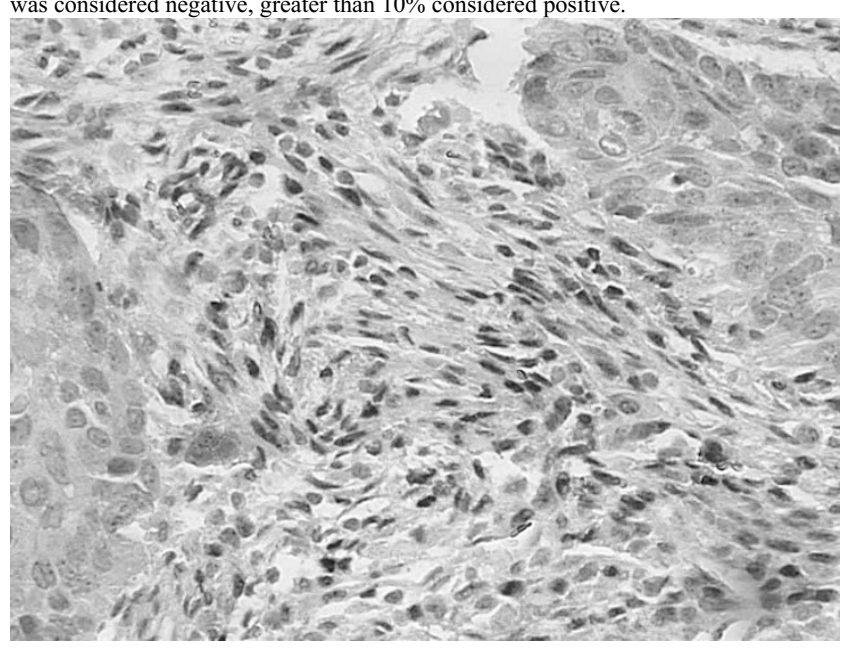

Correlation was measured between Twist results and the histologic subtypes.

Results: Kruskal-Wallis analysis on Twist positive cases showed a significant difference among five histologic subtypes $(\mathrm{P}<0.0001)$. The most common subtype was Acinar followed by Solid 
table1; Twist expression in defferent subtypes

\begin{tabular}{|l||l|l|}
\hline Histologic Subtypes & Twist + & Twist - \\
\hline Lepidic & 1 & 1 \\
\hline Acinar & 13 & 9 \\
\hline Solid & 10 & 7 \\
\hline Papillary & 6 & 2 \\
\hline Micropapillary & 5 & 1 \\
\hline
\end{tabular}

Chi-square analyses revealed a significant difference in Twist expression in cases that comprise greater than $20 \%$ of either or both of these two subtypes.

table2; Twist expression in Acinar and Solid

\begin{tabular}{|c|c|c|c|}
\hline Acinar \& Solid & Twist + & Twist - & P value \\
\hline$>60 \%$ & 9 & 6 & 0.03 \\
\hline$>40 \%$ & 16 & 7 & 0.03 \\
\hline$>20 \%$ & 17 & 9 & 0.03 \\
\hline
\end{tabular}

Conclusions: Twist expression is differentially expressed in the five recognized subtype of lung adenocarcinoma and may contribute to the biological variability and difference in prognosis observed for these subtypes.

2001 Pulmonary Vascular Changes Associated with Left-Sided Heart Disease (LHD) and Pulmonary Veno-Occlusive Disease (PVOD): A Comparative Morphometric Study

JJ Maleszewski, BT Larsen, SM Jenkins, PA Pellikka, JH Ryu, JE Yi. Mayo Clinic, Rochester, MN.

Background: LHD patients may have clinical and morphologic manifestations reminiscent of PVOD. To date, no study has systematically quantitated these changes with attention to the variable and distinguishing characteristics of these entities.

Design: Three age- and sex-matched cohorts were evaluated: normal $(\mathrm{n}=5)$, LHD $(\mathrm{n}=10)$, and PVOD $(\mathrm{n}=10)$. In each case, 10 vessels, including: 6 small pulmonary vessels ( $<200 \mu \mathrm{m}$ in diameter), 2 pulmonary arteries (200-600 $\mu \mathrm{m}$ in diameter), and 2 pulmonary veins (200-600 $\mu \mathrm{m}$ in diameter), were assessed. Percent intimal thickness (\%IT), \% medial thickness (\%MT), and adventitial thickness (AT) were measured. Hemodynamic data was retrospectively collected from echocardiograms obtained prior to tissue sampling. Measures were compared via regression models utilizing generalized estimating equations (multiple vessels per patient)

Results: The mean \%IT in small vessels in LHD was less than in PVOD (28.0\% vs $38.1 \%, p=0.01)$ and both were greater than in normal $(16.8 \%, p<0.001)$. The mean $\%$ IT in pulmonary arteries was also lower in the LHD cohort $(7.7 \%)$ than in the PVOD cohort $(17.1 \%)(p=0.008)$. There was a trend to lower $\%$ IT in pulmonary veins in LHD $(28.9 \%)$ than in PVOD $(41.3 \%)(p=0.08)$. The significance of all of these features was however eliminated when results were normalized to right ventricular systolic pressures (RVSP). \%MT in pulmonary arteries was lower in LHD (15.7\%) than in PVOD (27.6\%) or normal $(13.2 \%)(p<0.0001)$, though the difference in \%MT of the pulmonary veins was not as significant. Mean AT of pulmonary veins was also less in LHD than in PVOD ( $26.1 \mu \mathrm{m}$ vs $36.4 \mu \mathrm{m}, p=0.03$ ).

Conclusions: This study demonstrates distinct morphometric differences in LHD and PVOD patients, particularly in small vessels $(<200 \mu \mathrm{m}$ in diameter). These differences disappeared when the after results were normalized to RVSP, suggesting that the hemodynamic status is a primary determinant for pulmonary vascular pathology in LHD and PVOD.

\section{Localized Malignant Epithelioid Mesothelioma}

\section{SE Mann, CA Moran, N Kalhor. MD Anderson Cancer Center, Houston, TX.}

Background: Malignant Mesothelioma is an uncommon tumor that usually presents with diffuse pleural involvement and caries a dismal prognosis. Rarely, this tumor can present as a localized intraparenchymal mass or focal pleural lesion. The patient prognosis is variable, but is often better than the diffuse counterpart with some patients showing no recurrence of disease many years after surgery.

Design: A search of our pathology files yielded 1002 cases of malignant mesothelioma from $11 / 2005$ to $10 / 2013$. Only 4 cases of localized mesothelioma were identified, corresponding to $<1 \%$ of all mesotheliomas. The patient ages range from 42 to 78 years. Three cases are intrapulmonary masses with attachment to the visceral pleura. The fourth case showed pleural thickening found within a wedge resection for an adjacent neuroendocrine tumor. Clinically, the patients were asymptomatic related to their mesothelioma and in three cases, the intraparenchymal masses were identified radiologically for unrelated issues. In the fourth case, the focal pleural lesion adjacent to a neuroendocrine tumor was not identifed prior to surgery. History of asbestos exposure was not stated in any of the patient histories.

Results: Histologically the findings of three cases are epithelioid malignant mesothelioma characterized by nests of polyhedral cells with round nuclei and moderate eosinophilic cytoplasm and the fourth case is a sarcomatoid malignant mesothelioma with the classical features of pleomorphic spindle cells growing in short fascicles. All four cases were positive by immunohistochemical stain for anti-calretinin and for cytokeratin. The three epithelioid mesothelioma cases were positive for cytokeratin $5 / 6$ which was negative in the sarcomatoid malignant mesothelioma. One patient had a recurrence of tumor involving lung and diaphragm four years after the original workup and surgery. Two cases are very recent and a reasonable follow-up interval has not elapsed. One patient remains alive after 8 years with no recurrence of mesothelioma. Conclusions: Localized malignant mesothelioma is exceedingly rare compared to the diffuse type. These localized tumors share the same histologic and immunohistochemical profiles as the diffuse type, but differ radiologically and in clinical presentation. Although the course of disease is difficult to predict, some patients enjoy an extended disease-free interval compared to patients with diffuse malignant mesothelioma. These tumors can be diagnostic pitfall for the practicing pathologist. Awareness of this entity with a place in the differential diagnosis of solitary lung lesions is essential to avoid errors in diagnosis.
2003 Laminin Alpha-1 but Not Laminin Gamma-2 Accumulates in the Cytoplasm of Malignant Mesothelial Cells in the Progression from Epithelioid to Sarcomatoid Morphology

SMcGregor, R Dunning, AN Husain, W Vigneswaran, T Krausz. University of Chicago, Chicago, IL.

Background: Malignant pleural mesothelioma (MPM) can be epithelioid, biphasic and sarcomatoid. One can hypothesize that epithelioid cases evolve to sarcomatoid via a biphasic intermediate undergoing epithelial mesenchymal transition (EMT). EMT produces an invasive phenotype and is demonstrated by cytoplasmic laminin. EMT portends a poor prognosis in carcinoma and MPM, as seen by cytoplasmic laminin or sarcomatoid morphology, respectively. Given this parallel and the manner that normal mesothelial cells rest on basement membranes, we investigated laminin in MPM.

Design: We constructed a tissue microarray (TMA) of epithelioid $(n=33)$, biphasic $(n=18)$ and sarcomatoid $(n=11)$ MPM's. One epithelioid case was excluded due to tissue loss. H\&E and immunohistochemical stains for laminin alpha-1 (part of Ln-111 and Ln-121) and laminin gamma-2 (unique to Ln-332) were performed. Stains were scored for intensity $(0-3+)$ of cytoplasm.

Results: Laminin alpha-1 was intracellular in $93 \%$ of MPM cases. Staining intensity was lowest in epithelioid cases and highest in sarcomatoid. In contrast, laminin gamma-2 was expressed in $51 \%$ of MPM cases and showed no preferential pattern between the subtypes. Detailed results are shown in Figures 1 and 2 (E=Epithelioid, B-E=Epithelioid biphasic, B-S=Sarcomatoid biphasic, $\mathrm{S}=$ Sarcomatoid).

Laminin alpha-1
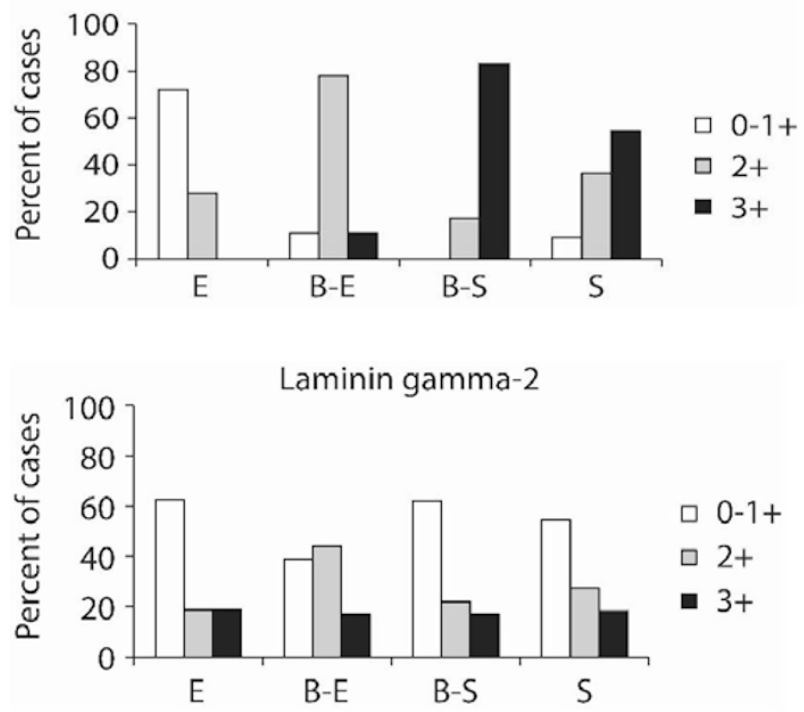

Conclusions: Both laminin alpha- 1 and laminin gamma- 2 are expressed in the cytoplasm of malignant mesothelial cells. However, whereas gamma-2 demonstrates no predilection according to morphologic subtype, alpha-1 steadily increases in intensity from pure epithelioid cases to the epithelioid component of biphasic cases and then even further sarcomatoid component of biphasic and pure sarcomatoid. Sarcomatoid morphology is known to portend the poorest prognosis in MPM patients and laminin alpha-1 staining may be useful prognostically.

2004 Combined Infiltrates of Conventional CD8+T-Cells, CD4+Foxp3+ Regulatory T-Cells and PD-1+ Lymphocytes Are Common in Malignant Pleural Mesothelioma

S McGregor, R Dunning, AN Husain, W Vigneswaran, T Krausz. University of Chicago, Chicago, IL.

Background: Mixed inflammatory infiltrates are a known feature of malignant pleural mesothelioma (MPM), but the subtleties of their composition are currently unclear. T-cells are highly diverse, with multiple subsets that may potentially be exploited for immunomodulatory therapy, including CD8+ cytotoxic T-cells, regulatory T-cells (Treg, CD4+Foxp3+) and those expressing programmed death-1 (PD-1). The current study was aimed at understanding the contribution of these various populations to MPM infiltrates. Design: Tissue microarrays (TMA) were constructed using epithelioid ( $\mathrm{n}=33$ ), biphasic $(\mathrm{n}=18)$ and sarcomatoid $(\mathrm{n}=11)$ MPM cases. Two epithelioid cases were excluded from analysis due to tissue loss. Routine H\&E staining, double immunohistochemical staining for Foxp3 with either CD4 or PD-1 and single staining for CD3 and CD8 were performed. CD3 and CD8 were scored as low (no cluster consisting of more than 10 cells) or high ( $>10$ cells in a given cluster). CD4+Foxp3+ and CD4+PD-1+ cells were scored according to the maximum number of double-stained cells per high power field (hpf, 400X)

Results: All MPM infiltrates demonstrated CD3+ and CD8+ T-cells. CD3+ infiltrates were low in $55 \%$ of cases and high in $45 \%$ of cases, while CD8+ T-cells were somewhat fewer, with $82 \%$ of cases scored as low and $18 \%$ scored as high. CD4+Foxp3+ and PD- $1+$ cells were present in $98 \%$ and $90 \%$ of cases, respectively, and $88 \%$ of cases demonstrated both populations. PD-1+ cells tended to outnumber CD4+Foxp3+ cells, with an overall average of up to 22.0 and 16.7 cells per hpf, respectively. PD-1+Foxp3+ cells were present in only $55 \%$ of cases, and when present, they were few (up to 0.9 cells per hpf on average). 
Conclusions: MPM cases demonstrate infiltrates containing multiple T-cell subsets within any given case, including Treg and PD-1+ lymphocytes in additional to conventional CD8+ T-cells. Immunomodulatory therapies targeting each of these populations are currently in development and have demonstrated success in murine models. It is tempting to speculate that the mixed nature of this infiltrate may afford the opportunity for synergistic effects from targeting multiple populations simultaneously.

2005 MET Status before Tyrosine Kinase Inhibitor (TKI)-Start Predicts Treatment Responses in EGFR-Mutant Lung Adenocarcinoma

A-SK Meyer, R Marienfeld, S Rudiger, P Muller, P Moller, C Schumann, JK Lennerz. University Ulm, Ulm, Germany; Hospital Guenzburg, Guenzburg, Germany.

Background: The type of EGFR mutation in non-small cell lung cancer (NSCLC) is predictive for tyrosine-kinase inhibitor (TKI) responses. TKI-resistance limits responses and among several oncogenic drivers, MET causes resistance in at least $30 \%$ of patients. MET-positive NSCLC is clinically more aggressive and the predictive influence of MET remains unclear in TKI-treated EGFR-mutant NSCLC. Here, we tested the METstatus as a biomarker for TKI-response in EGFR-mutant lung adenocarcinoma (ADC). Design: The inclusion criteria for this retrospective study were: $E G F R$-mutation within exon 18-21, histologically confirmed ADC (Stage III/IV), TKI-treatment for at least $1 \mathrm{mo}$., clinical follow-up and sufficient tissue for MET assessment by immunohistochemistry. Primary outcome measure was overall response to TKI (OR) at end of observation. Secondary outcome measures were best radiological response (BR, by CT, RECIST 1.1), progression free (PFS) and overall survival (OS). $\mathrm{P}<0.05$ denotes statistical significance.

Results: Of 812 genotyped Caucasian NSCLC patients, $74(9 \%)$ had EGFR mutations. Eighteen fulfilled the inclusion criteria and MET-positivity was present in 8 tumors (44\%). OR was progressive disease (PD) in 4 patients (MET: $2+; 2-$ ), stable disease in 9 patients (MET: $6+; 3-)$, and partial response (PR) in 5 patients who were all MET-(PR vs. not-PR; $P=0.05$; Fisher's exact test). Tumors of patients with $\mathrm{PR}$ had significantly lower MET-expression $(P=0.04 ; t$-test $)$ and MET status correlated significantly with $\mathrm{BR}\left(\mathrm{R}^{2}: 0.3 ; P=0.04\right)$. Patients with MET- tumors demonstrated significantly more tumor shrinkage to TKI (50.5\% MET-vs. $30 \% \mathrm{MET}+; P=0.02, t$-test $)$ and achieved their best response later (MET +2.5 months vs. MET -7 months; $P=0.03, \log$-rank). There was no association of MET with PFS or OS; however, patients with PD had the shortest PFS and OS $\left(P_{P F S}:<0.0001 ; P_{O S}:<0.0001 ; \log\right.$-rank).

Conclusions: Patients with MET-tumors show better responses than patients with MET+ tumors. While, the cohort size is a limitation, the stringent selection criteria are necessary to test the value of MET as biomarker in this specific setting. Given the statistically significant effect of MET status to TKI-response in our cohort, ongoing trials inhibiting both MET and EGFR, and the simplicity of the MET assessment, our findings should warrant validation in independent larger cohorts.

\section{EGFR Mutational Analysis in Non-Small Cell Lung Carcinomas} by Allele Specific PCR on Fine Needle Aspiration Derived Cell Pellets

EC Minca, J Brainard, S MacNamara, C Farver, PC Ma, AV Arrossi, NA Penell, RR Tubbs. Cleveland Clinic, Cleveland, $\mathrm{OH}$.

Background: Epidermal growth factor receptor (EGFR) gene mutation status in advanced non-small cell lung carcinomas (NSCLC) directs targeted therapy with EGFRspecific inhibitors, including erlotinib and gefitinib. EGFR mutations are commonly tested by Sanger sequencing or allele specific polymerase chain reaction (ASPCR) on formalin-fixed paraffin-embedded (FFPE) samples. Cytology specimens obtained by fine needle aspiration (FNA) represent an alternative valuable resource in cases when FFPE tissue is insufficient, unavailable or improper, which at our institution comprise approximately half of cases tested. Here we present the validation of ASPCR on FNA derived cells pellets and FFPE samples for detecting EGFR mutations in a NSCLC case series at our institution.

Design: The validation study included 100 samples from patients with advanced NSCLC clinically referred for EGFR mutation testing: 39 FNA cell pellets, 17 cytoblocks and 44 FFPE biopsies. ASPCR was performed using the EGFR RGQ PCR Kit (Qiagen) that detects 29 somatic EGFR mutations against a background of wild-type genomic DNA. DNA extracted and quantified from all samples was also sent for external testing at CLIA and CAP accredited facilities (Genzyme, Clarient, and Dahl-Chase).

Results: The ASPCR assay performed at our institution returned EGFR mutations in $35 / 100$ samples and wild type EGFR in 65/100 samples. The results were concordant with those of external testing in $98 / 100$ cases $(98 \%)$. The 2 discordants, involving 1 cytoblock and 1 FNA pellet, were interpreted as apparent false-positives, but may reflect a greater sensitivity of the in-house ASPCR assay. The assay had acceptable intra-assay, inter-assay and inter-lot reproducibility $(\% \mathrm{CV}<10 \%)$, and an analytical sensitivity level of $10 \%$ mutant allele. The clinical sensitivity, specificity, positive and negative predictive values were $100 \%, 94.3 \%, 97 \%$ and $100 \%$, respectively for the whole study, and $100 \%$, $87.5 \%, 96.9 \%$ and $100 \%$, respectively, for the FNA pellets alone.

Conclusions: ASPCR can reliably detect EGFR gene mutations in FNA cell pellets as in FFPE samples from patients with NSCLC. The high sensitivity of the assay in FNA specimens warrants the routine use of FNA cell pellets for clinical EGFR mutation testing on in NSCLC cases with unavailable or improper FFPE material.

2007 ALKTesting in Non-Small Cell Lung Carcinoma by FISH on Fine Needle Aspirate Derived ThinPrep Slides

EC Minca, C Lanigan, JP Reynolds, Z Wang, PC Ma, F Almeida, NA Pennell, RR Tubbs. Cleveland Clinic, Cleveland, $\mathrm{OH}$.

Background: Oncogenic $A L K$ gene rearrangements in non-small cell lung carcinomas (NSCLC) provide the basis for targeted therapy with crizotinib and other specific ALK inhibitors. Treatment eligibility is conventionally determined by the FDA-approved companion diagnostic fluorescence in-situ hybridization (FISH) assay on formalinfixed paraffin-embedded (FFPE) tissue. On limited samples such as fine needle aspiration (FNA)-derived cytoblocks, FFPE-FISH for $A L K$ is often uninformative due to insufficient tumor cells, absence of enumerable signals, or autofluorescence. FISH performed on liquid based ThinPrep slides may represent a robust alternative to conventional FFPE-FISH. Here we assessed the feasibility of $A L K$ testing by ThinPrepFISH in an extended NSCLC case series.

Design: ALK-status was tested on 230 FNA samples (ThinPrep slides and cytoblocks) from 217 patients with advanced NSCLC, including 179 consecutive samples collected from 174 patients between 12/2012 and 08/2013 at Cleveland Clinic. ThinPrep-FISH was performed using a standard $A L K$ break-apart probe set (Abbott Molecular Vysis) and scored in areas with abundant tumor cells etched with a diamond-tip pen on the reverse side of the slide. Due to the high failure rate of FFPE-FISH on cytoblocks ( $>30 \%$ in our preliminary experience), we used ultrasensitive ALK immunohistochemistry (IHC) on corresponding cytoblocks [D5F3 antibody (Cell Signaling) with OptiView signal amplification (Ventana)] as the reference data set (J Mol Diagn 15:341-6).

Results: Cytoblocks were unavailable in 19/230 (8.2\%) cases and lacked sufficient tumor cells in 57/230 (24.7\%) cases, despite the presence of abundant tumor cells on the ThinPrep slide. ALK ThinPrep-FISH was informative in 228/230 (99\%) cases, displaying robust signals, uncompromised by nuclear truncation inherent in FFPE-FISH. 9/178 (5\%) informative cases in the consecutive series and 18/228 (7.8\%) informative cases overall were $A L K$-rearranged. Of 154 informative matched ThinPrep-FISH and cytoblock-IHC samples, 152 were concordant (10, 6.5\% $A L K$-status positive; $142,92.2 \%$ ALK-status negative), and $2(1.3 \%)$ were ThinPrep-FISH positive but IHC negative (sensitivity $100 \%$, specificity $98.6 \%$, overall agreement $98.7 \%$ ).

Conclusions: $A L K$ [/italic]gene rearrangements are reliably detected by FISH using ThinPrep slides derived from minimally-invasive FNA cytopathology samples. ThinPrep-FISH can be confidently used for clinical ALK testing in NSCLC cases.

2008 Restrictive Allograft Syndrome and Idiopathic Pleuropulmonary Fibroelastosis: Same Histology for Two Different Scenarios

MA Montero-Fernandez, TOsadolor, VMonforte, A Bell, A Rice, AG Nicholson. Royal Brompton and Harefield NHS, London, United Kingdom; University Hospital Vall d'Hebron, Barcelona, Spain; Imperial College University, London, United Kingdom. Background: Restrictive Allograft Syndrome (RAS) and idiopathic pleuroparenchymal fibroelastosis (PPFE) are two different diseases that share the same histology. The former relates to chronic allograft dysfunction in lung transplantation with the latter being a rare condition in native lungs. Our aim is to compare their histology with biopsies of usual interstitial pneumonia (UIP) to look for injured target structures.

Design: We selected 8 post mortem recipient lungs from patients who died of RAS, 5 PPFE biopsies, 5 usual interstitial pneumonia biopsies and 5 sections of normal lung from the Histopathology Department archive files from Royal Brompton \& Harefield NHS Trust, London, UK and Vall d'Hebron University Hospital, Barcelona, Spain. Histopathological features were described, blinding the clinical and radiological features of each patient.

Results: Biopsies from RAS and PPFE showed subpleural, paraseptal and broncocentric fibroelatosis, with mild to moderate numbers of fibroblastic foci and inflammation. No microscopic honeycombing was noted. When comparing to UIP cases, there was a striking decrease of the interstitial capillary network (Fig.1B CD34 in PPFE/RAS and D. CD34 in UIP) as well as intimal fibrosis in medium size vessels (Figure 1C). Medial wall inflammation involving medium size vessels (arteries and veins) was seen in all patients diagnosed as RAS (Figure 1A)

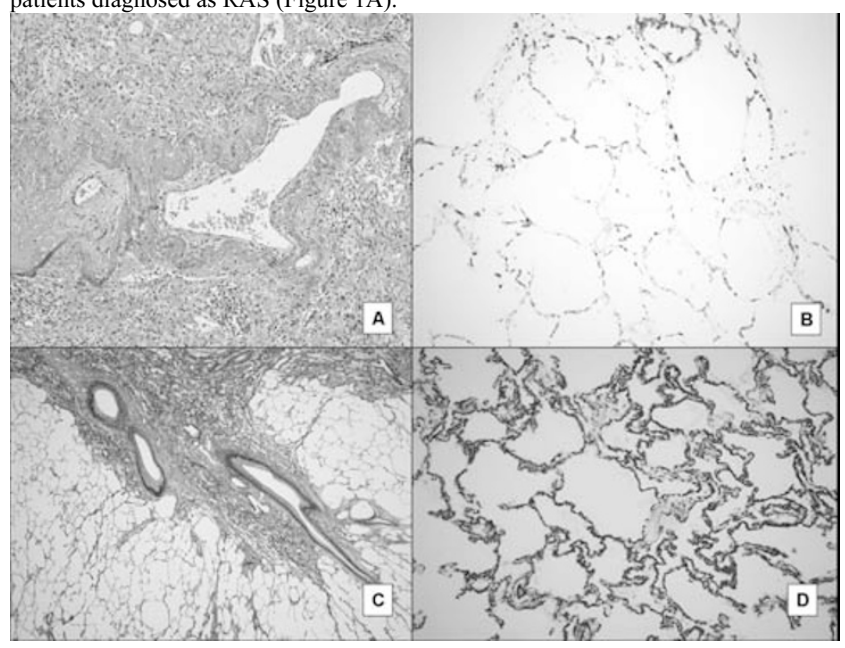

Conclusions: RAS and PPFE histopathology consisted of intra-alveolar fibrosis and elastosis with distinctive fibro-inflammatory vascular morphology and interstitial capillary loss. Although interstitial fibroelastosis have already been described in both diseases, we identify novel vascular changes, which may allow further understanding of their pathogenesis. 
2009 EGFR L858R Antibody Cross-Reaction in HER2 Positive Breast and Gastric Carcinomas

$V$ Montse, R Natalia, R Ruth, T Isabel, P Carme, A Nuria, M Begonya, G-P Beatriz, $P$ Xavier. Histopat Laboratoris, Barcelona, Spain; BIOPAT. Biopatolodia Molecular, Grup Assistencia, Barcelona, Spain; Hospital de Barcelona, SCIAS, Grup Assistencia, Barcelona, Spain.

Background: The use of epidermal growth factor receptor (EGFR) mutation-specific antibodies is likely to get soon incorporated into clinical practice due to its proven correlation with the presence of EGFR mutation in lung adenocarcinoma, especially in cases with limited tumor material, or in situations where molecular genetic analysis is not readily available. The use of these antibodies has also been suggested as an additional tool for distinguishing primary versus metastatic carcinomas in the lung. Incidental findings in our routine practice and a recently published paper reporting false positive breast carcinomas for EGFR L858R, made us think about the possible existence of a cross-reaction between HER2 and the EGFR L858R-specific antibody. Aims of this study were to further analyze the existence of this cross-reaction and its frequency in the two common sources of metastatic tumors to lung, breast and gastric cancer; in which, in addition, HER2 expression is widely studied in relation to targeted therapies. Design: The series consists of 55 primary tumors, 22 breast and 5 gastric carcinomas HER2 positive for overexpression and amplification, and 20 breast and 8 gastric carcinomas negative for both. EGFR mutations were studied by immunohistochemistry with two specific monoclonal antibodies (EGFR [E746_750del] clone 6B6 and EGFR L858R clone 43B2) in all cases, and confirmed using real-time PCR when positive. Results: In our study $86 \%$ (19/22) of HER2 positive breast carcinomas and $100 \%(5 / 5)$ of HER2 positive gastric carcinomas showed EGFR L858R positive expression, and equivocal in $14 \%(3 / 22)$ of HER 2 positive breast carcinomas, while no expression was found in none of the HER2 negative carcinomas. All cases were negative using the EGFR exon19 [E746_750del] antibody. Real-time PCR did not confirm the presence of the EGFR L858R mutation on neither of the 24 positives nor of the 3 equivocal cases by IHC. Conclusions: EGFR L858R antibody gives false positive results in most of breast and gastric carcinomas with HER2 overexpression/amplification. The existence of this cross-reaction makes necessary verify any EGFR L858R positivity by molecular methods and discard the presence of HER2 overexpression/amplification in order to establish its clinical significance.

2010 Significance of Morphological Heterogeneity of Lung Adenocarcinoma Evaluated by FFPE-Based miRNA Profile, in Comparison with Genotype

NMotoi, H Nagano, E Miyauchi, Y Saito, Y Sugiura, H Ninomiya, H Kanda, S Okumura $Y$ Ishikawa. The Cancer Institute, Japanese Foundation for Cancer Research, Tokyo, Japan; The Cancer Institute Hospital of JFCR, Japanese Foundation for Cancer Research, Tokyo, Japan.

Background: Concerning clinical assessment of lung cancer, molecular-based approach is getting more important. When we think based on morphological heterogeneity, response to molecular-target therapy might not be very simple. The goal of this study is to evaluate significance of the morphological heterogeneity and the gene alteration of lung cancer, using FFPE-based miRNA expression profile.

Design: 52 lung cancer, including 43 adenocarcinoma (Ad), 6 squamous cell carcinoma (Sq), and 3 pleomorphic carcinoma (Pleo), were recruited. Average age was 62-yo, 28 male and 24 female, 20 ALK-altered, 10 EGFR-mutated. Using the FFPE material of surgically resected specimen, the comprehensive miRNA expression profile was examined by SurePrint G3 human MicroRNA microarray, Rel.16.0 (Agilent). The representative area of morphological subtype (h_sub) based on a new IASLC/ATS/ ERS classification was micro-dissected. Total 122 samples (including 19 lepidic (lep), 23 papillary (pap), 25 acinar (aci), 10 micropapillary (mipp), 22 solid (sol), 1 mucinous (muc) h_sub Ad, 13 Sq, 5 Pleo, 4 normal lung) were submitted. Hierarchical clustering and differential expressed probes by moderated t-test were calculated using Genespring GX (ver 12.6) (Agilent)

Results: Unsupervised hierarchical clustering revealed three groups; cluster-1 EGFR Ad, cluster-2 Ad with any genotype, cluster-3 Sq and Pleo. ALK-altered Pleo was included in cluster-3. Supervised clustering revealed the difference among the conditions of histology, of genotype and of $h$ sub. The number of differential expressed miRNA probe between each category ( $\mathrm{p}$ value $<=0.05, \mathrm{FC}>=2.0$ ) was as follows; 34 between Ad and $\mathrm{Sq}(\mathrm{Ad} / \mathrm{Sq}), 38$ lep/aci, 26 lep/sol, 5 lep/pap, 3 lep/mipp, and none in all the remainder $\mathrm{h}$ sub combinations. The miRNA profile would be considered as the biological indicator of difference between each condition. 96 miRNAs (excluding 202 duplicated entities), such as hsa-miR-144*, hsa-miR-182, hsa-miR-139-5p, were differentiatel expressed among the $\mathrm{h} \_$sub conditions. Among the genotype condition, 77 miRNAs (excluding 9 duplicated entities), such as hsa-miR-921, hsa-miR-494, hsa-miR-3648 were differentially expressed.

Conclusions: Our study suggested biological significance of histomorphologic difference of lung cancer in addition to the genetic abnormalities. These differential expressed miRNAs could be diagnostic indicators and/or new therapeutic targets.

2011 Distinction between Synchronous Lung Primary Non-Small Cell Carcinoma and Intra-Pulmonary Metastasis Using Next-Generation Sequencing

SJ Murphy, M-C Aubry, FR Harris, G Halling, SH Johnson, S Terra, T Peikert, ES Yi, $Y$ Ping, DA Wigle, V George. Mayo Clinic, Rochester, MN.

Background: Up to $20 \%$ of patients with lung cancer will present with multifocal lung nodules. Staging, treatment and prognosis is predicated on classifying these lesions as either synchronous primaries or metastatic disease. When excised, the histologic comparison can aid in this determination. However, in most cases, only biopsies or radiologic determination is used to make this assessment. Our study aimed at making this distinction using large genomic rearrangements identified by Next Generation Sequencing. We hypothesized that metastatic tumors should have at least one shared somatic breakpoint resulting from these rearrangements while synchronous primaries should not share any.

Design: The study group included 11 patients with synchronous lung tumors of similar histologic type, pathology used to determine the likelihood of tumors being synchronous primaries versus metastasis. Control groups included: 4 patients with paired lung primary and distant metastasis, 3 patients with synchronous lung cancers of different histologic types and 4 patients with multiple blocks of tissue of a single lung cancer. Laser capture microdissected tumor and adjacent normal tissue were whole genome amplified. Next generation DNA sequencing using the Mate Pair protocol was used to analyze large genomic rearrangements and selected findings were validated by PCR. Results: All 1ary lung tumors and distant metastasis shared between 4- 85 breakpoints (46-89\% of detected breakpoints) and blocks of a same tumor shared between 4-276 (50-100\%). In contrast, the synchronous tumors of different histologic type did not share a single breakpoint (out of 39-162). For the study group, 4 cases were predicted to be synchronous primaries, 5 to be metastatic and 2 were considered indeterminate. For all cases of synchronous primaries, no shared breakpoint was identified (of 6-69 breakpoints) and 4 of the 5 metastasis showed between 4-85 shared breakpoints (46$89 \%$ ). For the 2 indeterminate cases, the genomic data suggested synchronous primaries with no shared genomic breakpoint identified. All selected breakpoints validated through PCR. Furthermore, there was not a single shared genomic breakpoint between tumors from different patients in both groups.

Conclusions: Large genomic rearrangement sequencing is a robust method to distinguish synchronous lung primaries from metastasis. Breakpoints are unique to patients and to patient tumors such that sharing a common breakpoint would indicate clonality. Performing this study in a larger group of patients and determine clinical feasibility will be important.

2012 Practical Utility of IMP3, GLUT-1, and EMA for Distinguishing Malignant Mesothelioma from Benign Mesothelial Proliferations: A Korea Nationwide Study

M-H Oh, S Chang, S-HJung, J Abdul-Ghafar, S-Y Ji, J Han, T-J Kim, M Eom, KY Kwon, SY Ha, YD Choi, CH Lee, Y Lee. Soonchunhyang University College of Medicine, Cheonan, Korea; Inje University Ilsan Paik Hospital, Goyang, Korea; Yonsei University Wonju College of Medicine, Wonju, Korea; The Cardiopulmonary Pathology Study Group of Korean Society of Pathologists, Seoul, Korea.

Background: The differentiation of malignant and benign mesothelial proliferations is crucial in determining patient care and prognosis. But, this distinction can be extremely difficult, particularly in small biopsies. Recently, insulin-like growth factor II mRNAbinding protein 3 (IMP3) and glucose transporter 1 (GLUT-1) have been reported as specific and sensitive markers in the distinction of malignant mesothelioma from benign mesothelial proliferations. The purpose of this study is to evaluate the utility of IMP3, GLUT-1, and epithelial membrane antigen (EMA) immunohistochemistry for distinguishing malignant mesothelioma from benign mesothelial proliferations. Design: We performed IHC for IMP3, GLUT-1 and EMA using tissue microarrays of 47 malignant pleural mesotheliomas, 41 peritoneal mesotheliomas and 35 adenomatoid tumors for benign mesothelial proliferation. The malignant pleural mesotheliomas cases (pleuropneumonectomy, $\mathrm{n}=20$; excision, $\mathrm{n}=27$ ) and malignant peritoneal mesothelioma (excision, $\mathrm{n}=41$ ) are selected from archives of Korea Nationwide Malignant Mesothelioma Surveillance tissue bank between 2002 and 2012. Cytoplasmic staining in $\geq 10 \%$ of target cells was considered positive for IMP 3 and EMA and membranous tatining in $>10 \%$ of target cells was considered positive for GLUT-1.

Results: Sensitivity and specificity of IMP3, GLUT-1, and EMA expression in malignant mesothelioma and benign mesothelial proliferation are summarized in Table 1.

Table 1. IMP3, GLUT-1, and EMA expression in malignant mesothelioma and benign mesothelial

\begin{tabular}{|c|c|c|c|c|c|c|}
\hline & $\begin{array}{l}\text { IMP3 } \\
(\mathbf{n}=87)\end{array}$ & $\begin{array}{l}\text { GLUT-1 } \\
(\mathrm{n}=87)\end{array}$ & $\begin{array}{l}\text { EMA } \\
(\mathrm{n}=\mathbf{8 8})\end{array}$ & $\begin{array}{l}\text { IMP3+GLUT-1 } \\
(\mathrm{n}=87)\end{array}$ & $\begin{array}{l}\text { IMP3+EMA } \\
(\mathrm{n}=\mathbf{8 8})\end{array}$ & $\begin{array}{l}\text { GLUT-1+EMA } \\
(\mathrm{n}=88)\end{array}$ \\
\hline Malignant & $32(37 \%)$ & $18(21 \%)$ & $36(41 \%)$ & $39(45 \%)$ & $58(66 \%)$ & $47(53 \%)$ \\
\hline $\begin{array}{l}\text { Benign } \\
(\mathbf{n}=\mathbf{3 5})\end{array}$ & $0(0 \%)$ & $0(0 \%)$ & $0(0 \%)$ & $0(0 \%)$ & $0(0 \%)$ & $0(0 \%)$ \\
\hline Sensitivity & $37 \%$ & $21 \%$ & $41 \%$ & $45 \%$ & $66 \%$ & $53 \%$ \\
\hline Specificity & $100 \%$ & $100 \%$ & $100 \%$ & $100 \%$ & $100 \%$ & $100 \%$ \\
\hline Pvalue & 0.0001 & 0.0085 & $<0.0001$ & $<0.0001$ & $<0.0001$ & $<0.0001$ \\
\hline
\end{tabular}

IMP3 and EMA staining showed diffuse and strong pattern. The GLUT-1 showed diffuse and strong staining for red blood cells and endothelial cells but patchy staining for tumor cells

Conclusions: Although IMP3, GLUT-1, and EMA expression was statistically significant in mesotheliomas compared with benign mesothelial proliferations, it is unsuitable as individual marker because of low sensitivity. Use of IMP3 and EMA together could improve sensitivity and help in distinguishing malignant from benign mesothelial proliferations. Combined panel of immunohistochemical stains could be a useful approach.

\section{Liposarcomas of the Posterior Mediastinum: A Clinicopathologic} Study of 16 Cases

POrtega, E Zambrano, CA Moran, CMorrison, S Suster. Medical College of Wisconsin, Milwaukee, WI; M.D. Anderson Cancer Center, Houston, TX; Roswell Park Cancer Center, Buffalo, NY.

Background: Liposarcomas originating in the mediastinum are rare tumors that have been described mainly in the anterior mediastinum; very little is known about their histologic features and biologic behavior when occurring in the posterior mediastinal compartment. 
Design: We have studied sixteen cases of liposarcomas (LPS) presenting in the posterior mediastinum. Immunohistochemical stains and fluorescence in-situ hybridization (FISH) were done in selected patients.

Results: Ten patients were men and six were women, aged 29 to 87 years (mean: 57). The tumors presented clinically as large lobulated masses that ranged from 8 to $30 \mathrm{~cm}$ in greatest diameter (median: 15). The most common symptoms were cough, dysphagia, chest pain and abdominal pain. Six patients were asymptomatic and the tumors were discovered incidentally on chest X-rays. Histologically, 10/16 cases were atypical lipomatous tumor/well differentiated liposarcoma; 3/16 pleomorphic liposarcoma, 2/16 de-differentiated liposarcoma and 1/16 myxoid liposarcoma. Immunohistochemistry for MDM-2, S-100 and MIB-1 was performed in only three cases; one case of atypical lipomatous tumor showed positivity for S-100 and MDM-2. FISH was performed in two cases of atypical lipomatous tumors and showed high levels of amplification of $M D M 2$ at $12 \mathrm{q} 13-15$ in two cases, whereas the $C H O P$ gene translocation was negative. Complete surgical excision was performed in ten cases; negative surgical margins were obtained in only four cases. Clinical follow up data ranging from 3 to 192 months (median 28 months) was available in 12 patients. Two patients died of tumor, one after 4 months and the second one after an open-and-close surgery (myxoid liposarcoma and pleomorphic liposarcoma, respectively). Four patients (4/10) were alive and well with no evidence of disease at last follow up (median: 11.8 months); five patients $(5 / 16)$ were alive with disease at last follow up (median: 47.8 months); and one patient (1/16) died of unrelated causes.

Conclusions: All major histologic subtypes of liposarcoma were represented in the posterior mediastinum. In general, the biologic behavior of liposarcoma in the posterior mediastinum seems to correlate well with the histologic subtype and mirrors that of their counterparts at other soft tissue sites.

2014 Histological Evaluation of Clinically Detected 44 Lung Dominant Connective Tissue Disease Cases

K Otani, T Tanaka, N Omote, K Kataoka, Y Kondoh, H Taniguchi, K Tabata, T Itoh, J Fukuoka. Kobe University Hospital, Kobe, Japan; Nagasaki University Graduate School of Biomedical Sciences, Nagasaki, Japan; Tosei General Hosipital, Seto, Japan. Background: Lung dominant connective tissue disease (LD-CTD) proposed by Fischer et al is defined provisionally as interstitial lung disease (ILD) having positive autoantibodies or histologic findings suggestive of CTD but without diagnosis of definite CTD. Their histological inclusion criteria have never been confirmed, therefore we evaluated cases of clinically defined LD-CTD if they fullfill the criteria. We also added some other histological findings possibly associated with CTD-ILD for the evaluation. With concern that rheumatoid arthritis and Sjögren's syndrome (RA/SjS) may show different characteristics from polymyositis/dermatomyositis and/or systemic sclerosis (PM/DM/SSc), we compared the histological findings between the two groups solely divided by autoantibodies.

Design: 44 cases fulfilling LD-CTD criteria with positive autoantibodies were selected and their pathological pattern, 4 histological criteria; lymphoid aggregates with germinal centers, plasmacytic infiltration, pleuritis, and perivascular fibrosis and 3 other findings; small airway disease, arterial intimal thickness, and presence of acute lung injury were evaluated. LD-CTD showing UIP pattern were compared with 23 IPF without autoantibodies (IPF-neg). LD-CTD with RA/SjS-related antibodies and those with $\mathrm{PM} / \mathrm{DM} / \mathrm{SSc}$-related antibodies were also compared. Chi square test was used for statistical analysis.

Results: 26 of LD-CTD showed UIP pattern. When compared with IPF-neg, lymphoid aggregates with germinal centers, plasmacytic infiltration and pleuritis were seen more frequently in UIP/LD-CTD $(50.0 \%$ vs. $4.3 \%, \mathrm{p}<0.01,73.1 \%$ vs. $39.1 \%, \mathrm{p}=0.02$, and $46.2 \%$ vs. $17.4 \%, p=0.03$ ). Other findings did not show significant differences. 16 of UIP/LD-CTD and 6 of IPF-neg fulfilled the histological criteria of LD-CTD. Any histologic findings reached significant difference between $\mathrm{RA} / \mathrm{SjS}$ suggestive cases and PM/DM/SSc suggestive cases. During follow up, 1 case of UIP/LD-CTD was diagnosed as definite CTD.

Conclusions: 3 out of Fischer's 4 criteria reasonably associated with clinical LD-CTD cases, although their specificity is not robust enough for criteria. Some of current IPF cases may fulfill the histological criteria of LD-CTD, however, clinical importance of such cases is not certain. Further large studies to evaluate clinical difference between IPF-neg and UIP/LD-CTD and reproducibility and importance of histological criteria are needed.

2015 EZH2 Overexpression as a Novel Prognostic Marker in Lung Adenocarcinoma

$V$ Parimi, R Raj, Y-H Chen, K Raparia. Northwestern University, Chicago, IL.

Background: Enhancer of zeste homolog 2 (EZH2) is the catalytic subunit of polycomb repressive complex 2 (PRC2) and mediates epigenetic silencing of target genes through trimethylation of lysine 27 on histone H3 (H3K27me3). EZH2 confers a proliferative advantage in tumor cells and is overexpressed in breast and prostate cancers and epigenetically silences tumor suppressor genes. Clinicopathological implications of EZH2 expression in lung adenocarcinoma has not been studied.

Design: EZH2, pEZH2, H3K27me3, PTEN and Ki-67 were evaluated in 162 patient samples of lung adenocarcinoma by immunohistochemistry (IHC) on formalin fixed paraffin embedded tissue microarrays. Immunostains were graded for nuclear intensity and percentages of positively stained tumor cells and divided into three groups: negative, low and strong expression. Pathologic and clinical characteristics were correlated and analyzed using appropriate parametric and nonparametric statistical methods.
Results: Of the 162 patients, 54 patients showed strong expression and 73 patients showed low expression of EZH2. Patients with expression of EZH2 comprised of 87 females and 40 males with mean age of 66 years. Clinical and pathological characteristics of patients with strong expression of EZH2 when compared to those with low/negative expression are summarized below.

Clinical and Pathological Characteristics

\begin{tabular}{|c|c|c|c|}
\hline Characteristic & EZH2 (strong) & EZH2 (low/negative) & p value \\
\hline Tumor size $(\mathrm{cm})$ & 2.9 & 2.1 & 0.04 \\
\hline Stage 1 & $20 \%$ & $66 \%$ & 0.0001 \\
\hline Stage 4 & $46 \%$ & $16 \%$ & 0.0001 \\
\hline Ki-67 index & $32 \%$ & $11 \%$ & 0.0001 \\
\hline Pleural Invasion & $24 \%$ & $7 \%$ & 0.015 \\
\hline Brain Metastasis & $15 \%$ & $3 \%$ & 0.015 \\
\hline Solid predominant pattern & $33 \%$ & $10 \%$ & 0.001 \\
\hline Loss of PTEN & $59 \%$ & $41 \%$ & 0.07 \\
\hline Survival (months) & 46 & 82 & 0.011 \\
\hline
\end{tabular}

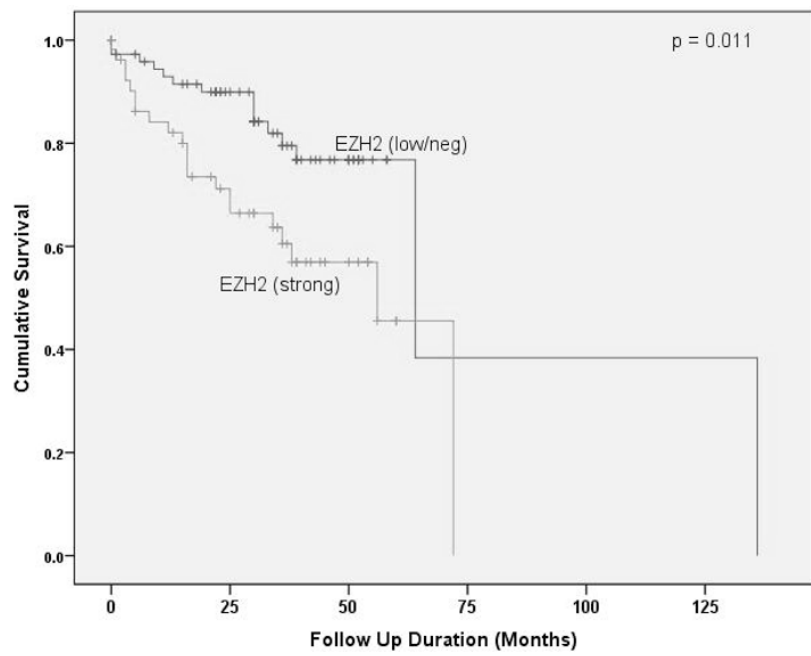

Conclusions: We demonstrate for the first time the correlation between EZH2 expression and several clinical and pathological characteristics in patients with lung adenocarcinoma. Strong expression of EZH2 is associated with larger tumor size, higher stage disease, higher proliferative index, brain metastases, pleural invasion and shortened survival. EZH2 overexpression can be a potential prognostic marker in lung adenocarcinoma.

2016 Is Transcription Factor GATA-3 Expressed in Malignant Mesotheliomas?

$S$ Patel, L-P Wang, Y Hung, F Valdivieso, A Ziober, L Litzky, M Feldman, C Deshpande. University of Pennsylvania, Philadelphia, PA.

Background: Zinc finger transcription factor GATA-3 is essential for early T-lineage progenitor development and controls T-helper type 2 (TH2) differentiation. Overexpression of GATA-3 has been reported in breast and urothelial carcinomas and is used in diagnostic surgical pathology to differentiate metastatic breast or urothelial carcinomas from prostatic or lung origin. In this study, we investigated the expression of GATA-3 in malignant mesotheliomas (MM) using tissue microarrays (TMAs).

Design: Tissue microarray was created using $0.6 \mathrm{~mm}$ tissue cores in triplicates from 94 diagnosed mesothelioma cases. GATA-3 antibody (Clone L50-823; Biocare, CA) was used and immunostaining was performed after heat-induced epitope retrieval. Nuclear immunoreactivity for GATA-3 antibody was scored (staining intensity and percentage positive cells) and at least $2+$ staining intensity in $>25 \%$ tumor cell nuclei was considered as positive staining. Appropriate tissue controls were used, and lymphocytes present were used as internal positive controls.

Results: In our study, 61 cases revealed presence of evaluable tissue cores; majority were pleural mesotheliomas $(54 / 61 ; 88 \%)$. 20 of these 61 cases $(33 \%)$ revealed positive nuclear staining for GATA-3. Fifteen of these were pleural mesotheliomas, 2 peritoneal mesotheliomas, 2 with both involved and 1 case where site was unknown. Nuclear GATA-3 immunoreactivity was noted in epithelioid (13/20 cases; $65 \%$, (Figure 1) and in sarcomatoid mesotheliomas (Figure 2). 


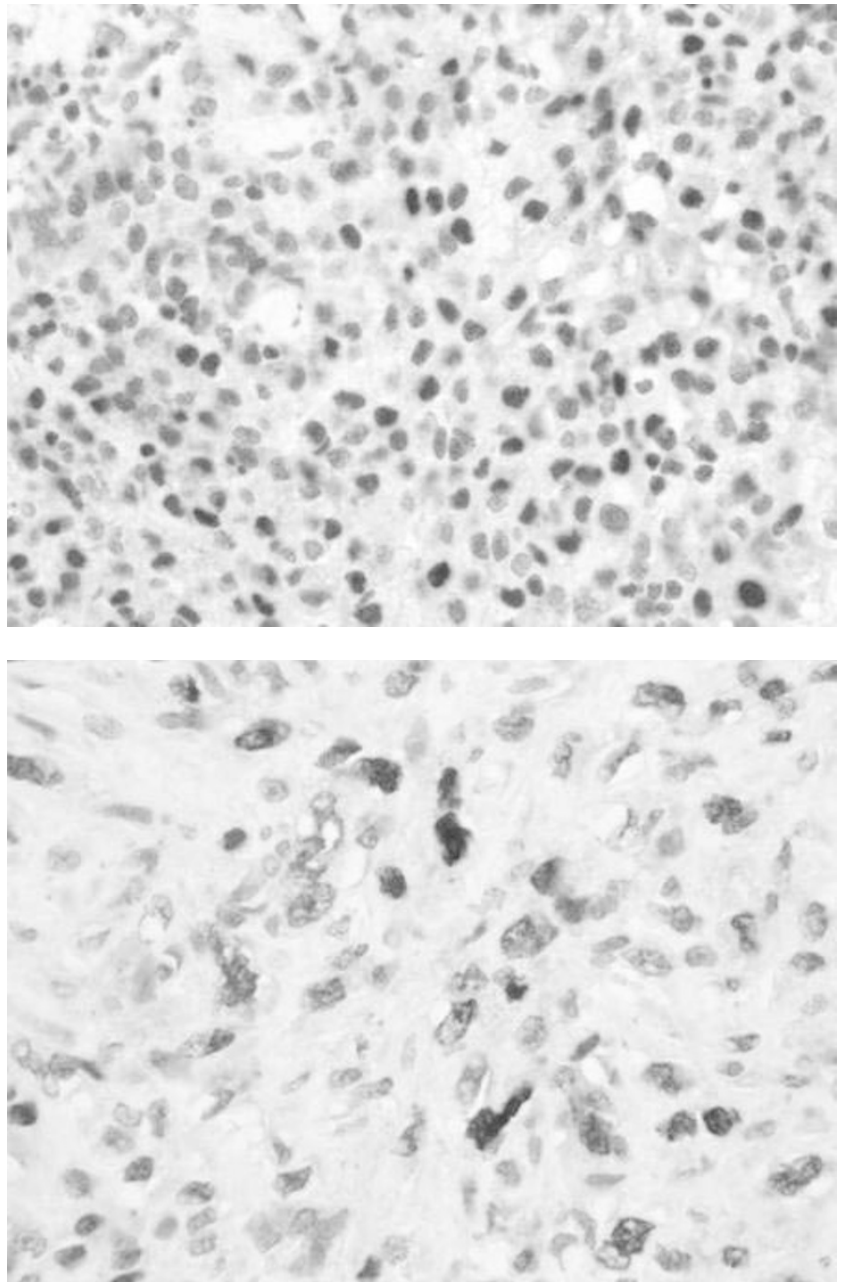

Conclusions: To date, GATA-3 positivity has not been reported in a series of mesotheliomas. Our study demonstrates GATA-3 expression in $25-30 \%$ of MM, including both epithelioid and sarcomatoid types. We also demonstrate that GATA3 can be expressed in peritoneal mesotheliomas. Currently GATA-3 is used in the workup of metastasis of unknown primary, and based on our findings, we suggest that mesotheliomas should also be included in the list of GATA-3 positive tumors along with urothelial and breast carcinomas.

2017 Morphology Outperforms Immunohistochemistry on Subtyping of NSCLC in Biopsies

$S$ Petkiewicz, H Sekhon, C Lai, P Wheatley-Price, M Gomes. University of Ottawa, The Ottawa Hospital, Ottawa, ON, Canada.

Background: Histological subtyping on biopsies of NSCLC is necessary for therapeutic purposes. Up to $50 \%$ of biopsies may have no diagnostic morphological features, and the 2011 IASLC classification suggests use of immunohistochemistry (IHC) for such cases. An IHC panel of TTF-1 and p63 is recommended for differentiating squamous (SCC) and adenocarcinoma (AC), however, there is evidence that $\mathrm{p} 40$ is more specific than $\mathrm{p} 63$ for diagnosing SCC.

Design: Paired biopsies and resections with NSCLC diagnoses were selected (37 cases). Using strict IASLC morphological criteria, two lung pathologists independently reviewed the slides and rendered diagnoses. Consensus diagnosis was made for any cases with disagreement. Cases that could not be diagnosed on morphology alone were designated as "NSCLC." IHC and special stains were performed on select slides from both biopsies and resections (TTF-1, mucicarmine, p63, p40). Staining was coded using IASLC criteria and the results compared with the morphology-only diagnoses. Results: Every resection showed either squamous or glandular morphological differentiation ( $\mathrm{AC}, \mathrm{n}=25 ; \mathrm{SCC}, \mathrm{n}=12$ ). Using resection diagnoses as the gold standard, specific diagnoses were rendered on $92 \%$ of biopsies using morphology alone, and those diagnoses, after consensus, concurred with resection diagnoses in $100 \%$ of cases, yielding sensitivities (sens) of $92 \%$ and specificities (spec) of $100 \%$ for both SCC and AC. For IHC staining panels, TTF- $1+/$ p 63 - had sens $=60 \%$ and spec $=100 \%$ while TTF$1+/ \mathrm{p} 40-$ had sens $=76 \%$ and spec $=100 \%$ for AC. For SCC, p $63+/$ TTF $-1-$ had sens $=82 \%$ and $\mathrm{spec}=96 \%$ while $\mathrm{p} 40+/ \mathrm{TTF}-1$ - had sens $=90 \%$ and $\mathrm{spec}=96 \%$. Mucicarmine had sens $=32 \%$ and $\mathrm{spec}=82 \%$ for diagnosis of AC on biopsy. In the three cases in which morphology was not sufficient for a final diagnosis, IHC directed a diagnosis consistent with the resection in 2 cases and the third case, an AC, had insufficient tissue for TTF-1 staining but mucicarmine was positive and p63 and p40 were negative.

Conclusions: Morphology outperformed IHC alone in diagnosing lung cancer biopsies. Replacing careful morphological examination with IHC or use of IHC in cases which show diagnostic morphology may be misleading, time consuming and exhaust tissue.
IHC is a valuable tool in cases with no morphological differentiation. A simple panel of TTF- 1 and one squamous marker is sufficient, $\mathrm{p} 40$ being more specific than $\mathrm{p} 63$. For $\mathrm{AC}$, mucicarmine has poor sensitivity but may be helpful in porly differentiated cases.

2018 Prognostic Significance of ROS1 Protein Expression in Non Small Cell Lung Cancers

MJ Presta, CB Sheehan, CE Sheehan, JS Ross. Albany Medical College, Albany, NY. Background: $R O S I$ is an orphan receptor tyrosine kinase of the insulin receptor family. Although oncogenic fusions of ROS1 to other genes such as Ezrin1, CD74 and SCD4 have recently emerged as targets for targeted kinase inhibitor therapy in NSCLC, ROS1 protein expression has not, to date, been evaluated as a prognostic factor for the disease. Design: Formalin-fixed, paraffin-embedded sections from 129 NSCLC, including 36 squamous cell carcinomas (SCC), 59 adenocarcinomas (AC), and 34 bronchoalveolar carcinomas (BAC), were immunostained by a manual method with ROS1 (D4D6) rabbit monoclonal antibody (Cell Signaling Technology, Danvers, MA). The staining pattern was semiquantitatively assessed based on staining intensity and distribution and the results were correlated with morphologic and prognostic variables.

Results: Immunoreactivity for ROS1 was predominately cytoplasmic. ROS1 overexpression was noted in 20/129 (15\%) tumors, while strong diffuse expression was noted in $5 / 129(4 \%)$ tumors. ROS1 overexpression correlated with tumor type [32\% BAC vs $15 \%$ AC vs $0 \%$ SCC, $p=0.001]$; with tumor grade overall $[23 \%$ well vs $17 \%$ moderately vs $0 \%$ poorly differentiated, $\mathrm{p}=0.032$ ] and within the $\mathrm{AC}$ subgroup [ $8 \%$ well vs $29 \%$ moderately vs $0 \%$ poorly differentiated, $\mathrm{p}=0.038$ ]; with tumor stage overall [ $19 \%$ early vs $0 \%$ advanced, $\mathrm{p}=0.028]$, with a trend within the AC subgroup $[20 \%$ early vs $0 \%$ advanced, $\mathrm{p}=0.117]$. Within the $\mathrm{BAC}$ subgroup, there was a trend toward correlation with tumor size $[60 \%>3.0 \mathrm{~cm}$ vs $25 \%<=3.0 \mathrm{~cm}, \mathrm{p}=0.061]$. Conclusions: Cytoplasmic overexpression of ROS1 in NSCLC correlates with tumor type, grade and stage and is strongly associated with bronchiolo-alveolar (lepidic) carcinoma and adenocarcinoma subtypes. ROS1 expression is linked to welldifferentiated and moderately-differentiated tumors and is associated with early stage disease. Further study of ROS1 expression as a prognostic factor and potential target for therapy in NSCLC appears warranted.

\section{Clonality Studies in Nodular Amyloid Deposition}

E Racila, J Tong, P Starostik, T DeFedericis, M Merzianu. Roswell Park Cancer Institute, Buffalo, NY

Background: Nodular amyloid deposition (NAD, "amyloidoma") is a rare disease defined by mass-forming deposition of amyloid. Few studies of NAD exist to date, its pathogenesis is incompletely understood and molecular studies are rarely undertaken in clinical practice. We aim here to characterize NAD clinical and pathologic features, to retrospectively classify the underlying disorder, and to evaluate the utility of molecular studies in patients with NAD.

Design: Eighteen patients were identified with amorphous nodular deposits in our files; 2 were reclassified as light chain deposition disease and 4 were eliminated due to insufficient material/information. Clinical-radiological information, histo-morphologic findings of the index lesion and bone marrow, and laboratory studies including serum or urine protein electrophoresis (SPEP/UPEP) and immunofixation (SIFE/UIFE), molecular studies (PCR B-cell clonality using IgH FR1-3, IgK primes) performed on bone marrow, blood and/or tissue specimens were reviewed. All clinical, pathologic and molecular information was integrated at review.

Results: Among the twelve patients, six were men and six women. The average age at diagnosis was 62.6 years (range 35 to 87 years). The location of nodular amyloid was lung ( 6 cases), brain ( 2 cases), soft tissue ( 3 cases) and pharynx ( 1 case). The main lesion size ranged from 0.9 to $7 \mathrm{~cm}$ ( $2.5 \mathrm{~cm}$ average). Three patients $(25 \%)$ had concurrent autoimmune connective tissue disease. All NADs showed sparse lymphoplasmacytic infiltrate and a diagnosis of lymphoma or multiple myeloma was suggested in only three patients $(25 \%)$ at the time of original report. Five cases were originally assigned to surgical pathology and seven cases to hematopathology. Eight of the twelve tumors tested demonstrated evidence of B-cell clonality either by kappa/lambda IHC ( 6 cases) and/or PCR ( 6 cases) in the amyloid deposition samples. These tests were concordant in only two cases. Bone marrow clonality was present in most cases tested (5 of 6 cases). Four cases showed no evidence of clonality. SPEP/SIFE showed a monoclonal protein in only three of twelve cases. Seven clonal cases were classified as low grade B cell lymphoma with plasma cell differentiation, while one patient had a plasma cell neoplasm. No patient had evidence of systemic amyloidosis at first diagnosis.

Conclusions: The underlying disease in most NAD is a clonal B-cell expansion or neoplasm with plasma cell differentiation which is difficult to diagnose due to paucicellular lymphoplasmacytic infiltrate. Clonality studies should be performed and systemic work-up suggested when reporting NAD.

2020 Loss of PTEN Expression Is Associated with Increased Tumor Size and Higher Stage Disease in Patients with Lung Adenocarcinoma

H Rahn, V Parimi, R Raj, A Rosca, K Raparia. Northwestern University, Chicago, IL. Background: Mutation of the tumor suppressor gene phosphatase and tensin homologue (PTEN) is seen in various malignancies. PTEN encodes a dual specificity protein phosphatase that negatively regulates phosphatidylinositol 3-kinase/AKT signaling, a pathway with an established role in promoting cell cycle progression and survival. We evaluated the expression of PTEN in lung adenocarcinoma and correlated with clinical, pathological and molecular features.

Design: Tissue micro-arrays containing 162 lung adenocarcinoma samples in triplicates were immunostained with anti-PTEN and anti-MIB antibodies. PTEN expression in the tumor was graded for intensity (negative: 0; mild: 1; moderate: 2 and strong: 3 ) and percentage of tumor cells positively stained. Less than $10 \%$ tumor cells with 
mild nuclear/cytoplasmic staining were considered having loss of PTEN. All patients with lung adenocarcinoma were followed from 0 to 136 months (median 30 months). Pathologic and clinical characteristics were correlated and analyzed using appropriate parametric and nonparametric statistical methods.

Results: Seventy six patients $(47 \%)$ had loss of PTEN expression. These patients included $47(62 \%)$ females and $29(38 \%)$ males with mean age of 66 years (range 43 to 91 years). Patients with loss of PTEN had larger tumors $(2.9 \mathrm{~cm}$ versus $2.1 \mathrm{~cm}$, $\mathrm{p}=0.004)$ (Figure 1) and were less likely to have lepidic predominant adenocarcinoma ( $8 \%$ versus $23 \%, \mathrm{p}=0.07$ )

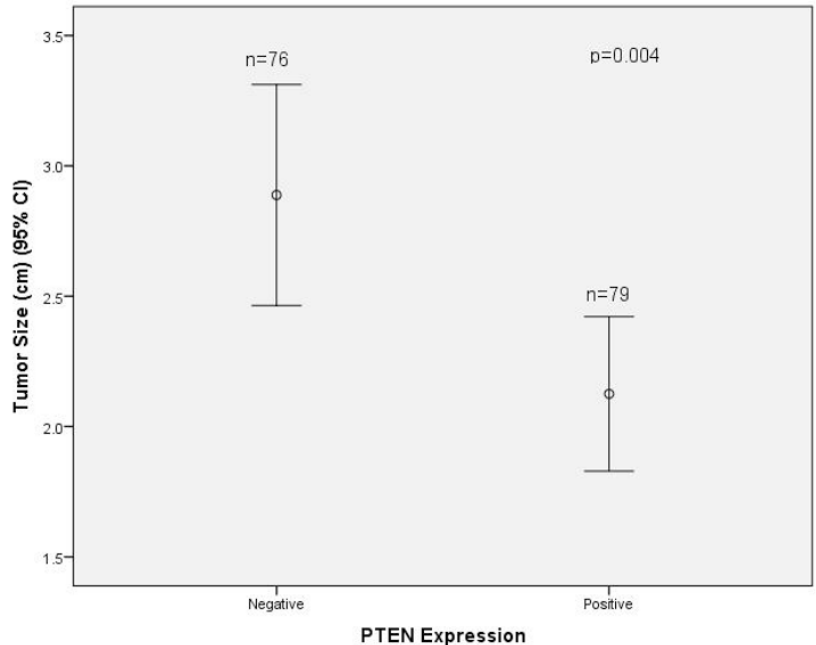

Patients with loss of PTEN expression were more likely to have a higher stage disease $(\mathrm{p}=0.006)$. Higher Ki-67 proliferative index showed a modest correlation with loss of PTEN expression ( $\mathrm{p}=0.12$ ). PTEN loss was seen in 24 patients with KRAS mutation and 10 patients with EGFR mutation.

Conclusions: Loss of PTEN protein expression occurs frequently in lung adenocarcinoma and portends a poor prognosis in terms of its association with increased tumor size, higher proliferative index and higher stage lung cancer.

2021 Hybrid Sarcoidosis and UIP - Sarcoidosis Variant or Distinct Histopathologic Entity?

N Rao, JF Tomashefski. Medical College of Wisconsin, Milwaukee, WI; Metrohealth Medical Center/Case Western Reserve University, Cleveland, $\mathrm{OH}$.

Background: Pulmonary sarcoidosis may progress to advanced interstitial fibrosis. Some end stage sarcoidosis features may be distinctive; however, other fibrosing conditions with granulomas, notably chronic hypersensitivity pneumonitis (HP), may pose diagnostic challenges. We present five cases of granulomatous lung disease consistent with sarcoidosis, spatially separated from a UIP pattern in adjacent parenchyma. This dual lung injury pattern appears distinctive from fibrotic phase sarcoidosis, and chronic HP, and warrants separate recognition and further study.

Design: 5 cases of a granulomatous process consistent with sarcoidosis, with background advanced interstitial fibrosis form the study. Clinical information was available in $4 / 5$ cases - 2 males and 2 females, 50 - 55 years old (mean age - 52). All presented with dry cough and dyspnea. Chest CT showed interstitial fibrosis with peripheral/axial involvement (1/4), peripheral honeycombing and traction bronchiectasis $(2 / 4)$, and lung base fibrosis (1/4). 4 patients had surgical lung biopsies; the 5 th underwent lung biopsy followed by lung transplantation. Follow up was available in $2 / 5$ patients. One is alive and well 5 years after diagnosis, following lung transplantation; the other has died of disease 5 years after diagnosis.

Results: Well circumscribed granulomas with characteristic lymphatic distribution, patchwork fibrosis, architectural remodeling and fibroblastic foci were present in all cases. One case showed end stage lung with advanced fibrosis. All cases had characteristic separation of granulomatous foci from adjacent UIP pattern fibrosis (Fig 1).

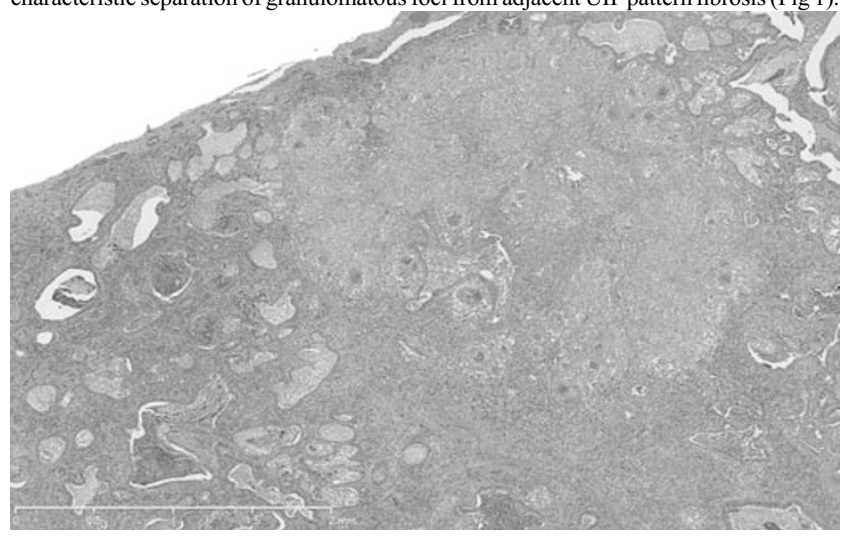

Mediastinal lymph nodes ( 2 cases), showed diffuse involvement by granulomatous inflammation with associated sclerosis.
Conclusions: We have characterized the pathologic features of 5 cases of granulomatous disease consistent with pulmonary sarcoidosis with synchronous UIP. The histologic features and distribution of granulomas, their spatial separation from UIP, and granulomas in mediastinal lymph nodes, should allow separation from chronic HP. While these findings may represent a sarcoidosis variant, coexistence of sarcoidosis and UIP cannot be excluded.

2022 IgG4 Positive Plasma Cell Infiltration in Lung Adenocarcinoma Is Common and May Be a Marker of Anti-Tumorigenic Immune Response $K$ Raparia, V Parimi, R Raj. Northwestern University, Chicago, IL.

Background: IgG4 related disease can be associated with lung adenocarcinoma. Significance of IgG4 plasma cell infiltrate in lung cancer is not well understood. Although few case reports and small case series describe IgG4 positive plasma cell infiltration in tumor stroma of lung cancer patients, the degree and frequency of IgG4 plasma cell infiltrate in patients with lung cancer has not been systematically studied. Design: Tissue microarrays containing 162 lung adenocarcinoma samples in triplicates were immunostained with anti-IgG4 and anti-IgG antibodies. Pathologic and clinical characteristics were analyzed and correlated.

Results: $43 \%$ patients ( $\mathrm{n}=70)$ had IgG4 positive plasma cells/HPF ranging from 1 to 65 (mean 5.3) (Figure 1) and IgG4/IgG ratio of 1.25 to $100 \%$ (mean $29 \%$ ) (Figure 2).
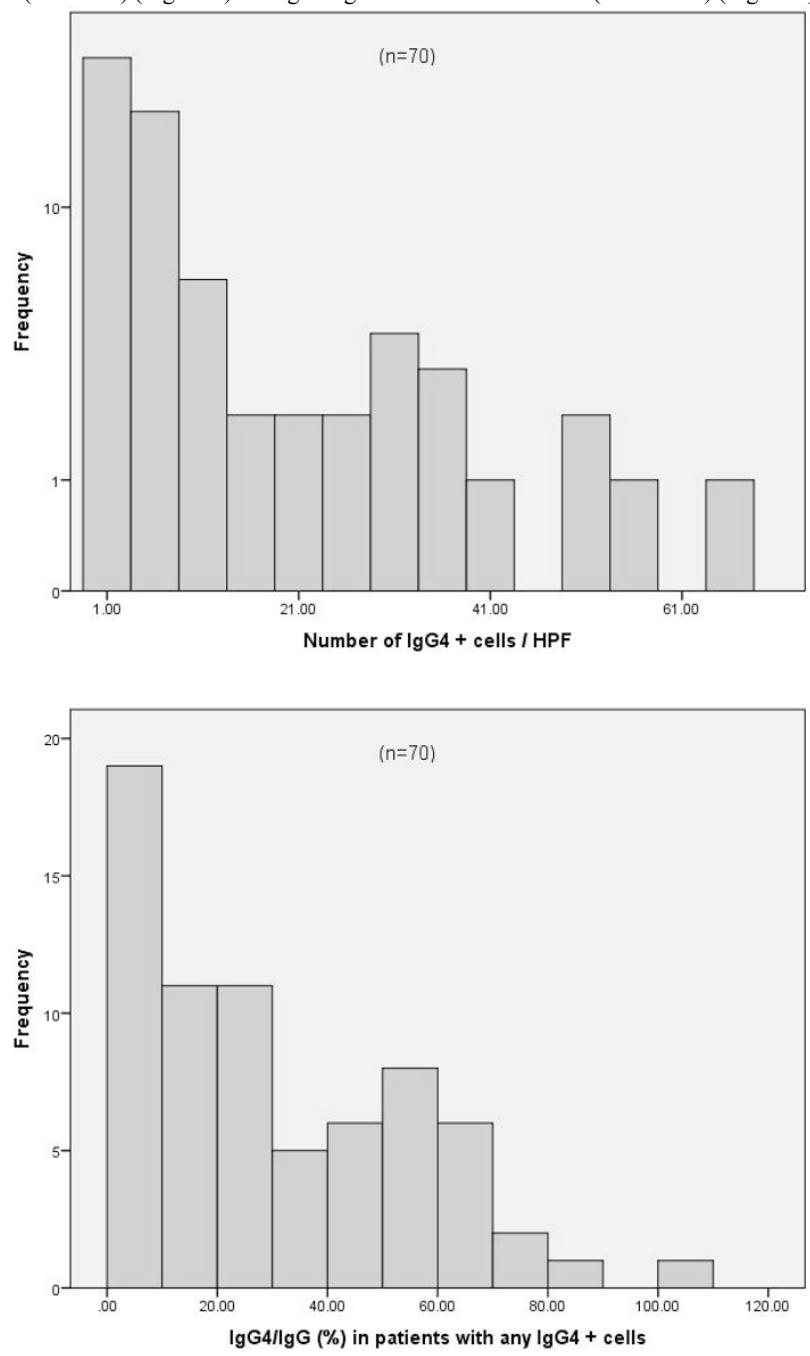

(10\%) tumors had more than $20 \mathrm{IgG} 4$ positive plasma cells/HPF and more than $25 \%$ IgG4/IgG cells (designated IgG4 enriched tumors). Mean IgG4/IgG ratio in the IgG4 enriched tumors was $58 \%(25-100 \%)$. Patient characteristics of IgG4 enriched cases: 10 females, 6 males, median age 66 years and mean tumor size $2.8 \mathrm{~cm}$ (range 0.8 to $8 \mathrm{~cm})$. All the patients with IgG4 enriched plasma cells with Stage 1 disease were alive at the end of median follow up of 36 months.

Conclusions: IgG4 positive plasma cell infiltration is relatively common in lung adenocarcinoma, with a minority of patients meeting diagnostic criteria for IgG4 related disease suggesting that the IgG4 positivity may represent an immune response to yet unidentified tumor antigens. There was a trend towards better survival in patients with IgG4 enriched tumors, suggesting that this immune response has anti-tumorigenic properties and could confer a survival advantage. This needs to be further confirmed in larger prospective studies. 
2023 Histopathologic, Immunophenotypic and Cytogenetic Features of Pulmonary Mucoepidermoid Carcinoma

AC Roden, JJ Garcia, RN Wehrs, TV Colby, KO Leslie, L Chen. Mayo Clinic Rochester, Rochester, MN; Mayo Clinic Arizona, Scottsdale, AZ.

Background: Mucoepidermoid carcinomas (MEC) are rare primary lung neoplasms. MEC has been extensively studied at other anatomic sites; however, few studies have attempted to characterize the histologic and immunophenotypic features of pulmonary MEC (PMEC). There is a strong association between MEC and $t(11 ; 19)$ (q21;p13); a recent study showed MAML2 rearrangement in 77\% of PMEC. Because this translocation is considered disease-defining for MEC, its significance in PMEC warrants further study.

Design: Clinical and consultation files at Mayo Clinic Arizona and Rochester were searched for PMEC. 4 of 21 identified cases were previously reported (Molina et al 2007). All cases were re-reviewed and graded according to Brandwein et al. Consecutive slides were stained with TTF-1, napsin A, p40 and p63. Staining was scored: 0 (neg), 1 (1-25\% tumor cells), 2 (26-50\%), 3 (51-75\%), 4 (>75\%). FISH to detect MAML2 rearrangement used a $M A M L 2-11 \mathrm{q} 21$ break apart probe.

Results: 21 PMEC, classified as low $(n=4)$, intermediate $(n=13)$, or high grade $(n=4)$ contained a variable number of mucous, squamous and intermediate cells. Most PMEC were invasive as small nests and islands ( $\mathrm{n}=15$; invasion evaluable in 20 cases). An intracystic component $<25 \%$ was found in 6 and pronounced nuclear atypia and perineural invasion in 1 each. All cases with available gross description $(n=10)$ had a central/endo- or peribronchial location. Tumor-associated lymphoid population was present in 19 (of 21) cases. Tissue blocks were available in 12 cases. The distribution of p63 and p40 staining in tumor cells was concordant in all cases except one (p63 score- 4 , p40 score- 3$)$. Scores for p63 and p40 were $1(n=5), 2(n=2), 3(n=1)$ and 4 $(\mathrm{n}=3)$. TTF-1 and napsin were neg in tumor cells in all cases. MAML2 rearrangement was identified in all cases tested including low $(n=3)$, intermediate $(n=8)$, and high grade $(\mathrm{n}=1)$ PMEC. Clinical history was available in $11 \mathrm{pts}: \mathrm{M}: \mathrm{F}=7: 4$ (median age $48 \mathrm{yrs}$, range, 32-75). F/u was available in 9 pts (median f/u 5.3 years, range, 1.1-13.8). One pt died of unknown causes, one developed metastatic MEC.

Conclusions: The majority of PMEC are intermediate grade histologically. Features helpful in distinguishing this special form of lung cancer from others include central/ endo- or peribronchial location together with $\mathrm{p} 40$ and p63 expression, lack of TTF-1 and napsin expression and MAML2 rearrangement. Morphologic characteristics of PMEC that may assist in distinguishing it from squamous cell carcinoma include nested or solid growth pattern, mucous cells and lack of keratinization.

2024 Cell Signaling Factors in Early Stage Non Small Cell Lung Cancer (NSCLC): A Preliminary Study

I Sansano, ML Botero, T Moline, MT Salcedo, A Martinez, NMurtra, SRamon y Cajal. HU Vall d'Hebron, Barcelona, Spain.

Background: Early diagnosis is still the most important prognostic factor in NSCLC. Despite that, up to $30 \%$ of these patients recur. The aim of this study was to identify oncogenic alterations that can help to understand the molecular biology of this heterogeneous group of tumors.

Design: We retrospectively analyzed a cohort of 52 patients with NSCLC diagnosed at stage I from 1995 to 2011 . We recorded the histopathological characteristics of these tumors listed in each pathology report (histologic type, size, vascular/pleural invasion) and obtained follow up of 46 patients. We studied total and phosphorilated forms of 4E-BP1, eIF4E, mTOR, MAPK, S6 and YB1 by immunohistochemestry in paraffin-embedded whole sections. Levels of expression were semiquantitatively evaluated paying attention to intensity and percentage of staining in both nucleus and cytoplasm (hscore). For the statistical analyses we performed Kruskal-Wallis and Kaplan-Meier tests.

Results: The mean age was 63.12 years, male/female ratio was $42 / 10$ and diagnoses included 18 squamous cell carcinomas (SQ) and 36 adenocarcinomas (ADC). We observed statistically significant higher expression of 4E-BP1 and p4E-BP1 in SQ compared to $\mathrm{ADC}(\mathrm{p}<0.001$ and 0,002$)$. pmTor and $\mathrm{pS} 6$ in contrast, were higher in $\mathrm{ADC}$ $(\mathrm{p}<0.001$ and $=0.04)$. We did not observe any significant relation between expression and size or pleura/vascular invasion. 9 out of 46 (19.56\%) recurred (4 SQ and 5 ADC, 1 Tis, $7 \mathrm{~T} 1$ and $1 \mathrm{~T} 2$ ). Kaplan-Meier test showed statististically significant longer period of disease-free survival (DFS) for patients with lower expression of pmapk and no relation to other tumor characteristics.

Conclusions: We have found differences in cell signaling factors expression between early stage SQ and ADC and association between some of these factors and DFS. Larger series studies will be carried out to find out the prognostic or predictive value of these cell signaling factors.

2025 Pulmonary Adenocarcinoma In Situ Is Not Simply a Precursor of Invasive Tumor: Evidence by Status of Smoking, Driver Mutations and Receptor Tyrosine Kinase Pathway Activation

$S$ Sato, $N$ Motoi, S Okumura, M Tsuchida, O Matsubara, Y Ishikawa. The Cancer Institute, Japanese Foundation for Cancer Research, Tokyo, Japan; Niigata University, Niigata, Japan.

Background: Lung adenocarcinoma, especially adenocarcinoma in situ (AIS), often occurs in individuals who have never smoked, although smoking is the main cause of lung cancer.

Design: To characterize AIS and, in particular, determine how AIS might be related to smoking, we collected a large number of AIS cases and examined clinicopathological features, EGFR and KRAS mutation status, and activation status of receptor tyrosine kinase (RTK) downstream signal pathways, including pAkt, pERK and pStat3, using immunohistochemistry.
Results: We identified 111 AIS among 1,549 adenocarcinomas resected surgically during 1995-2010. There was a higher ratio of female non-smokers, but not male nonsmokers, in AIS compared with invasive adenocarcinomas $(\mathrm{p}<0.001)$. Smoking was not associated with other clinicopathological factors including expression of TTF-1, a cell lineage marker. EGFR mutations were identified in 41 of $71(58 \%)$ non-smokers and 19 of $31(61 \%)$ smokers, and KRAS mutations were in one of $71(1.4 \%)$ nonsmokers and two of $34(5.9 \%)$ smokers. For pAkt and pERK, around $40-50 \%$ of AIS were positive, and for $\mathrm{pStat} 3$, over $80 \%$ were positive, with no significant differences in either for activation rates between non-smokers and smokers. Mucinous AIS rarely harbored KRAS mutations. Taken together, compared with invasive counterparts, AIS occurs predominantly in females and non-smokers. However, characteristics of AIS arising in non-smokers and smokers were similar in terms of cell lineage, driver mutations and RTK pathway activation.

Conclusions: Our results suggest that AIS is not simply a precursor of invasive adenocarcinoma and that smoking may not be a cause of AIS. Rather, smoking may play a role inprogression of AIS to invasive adenocarcinoma with AIS features.

2026 Incidence of Unexpected Clinically Significant Findings in Spontaneous Pneumothorax Specimens: Is Routine Histologic Examination Justified?

JL Sauter, KJ Butnor. The University of Vermont/Fletcher Allen Health Care, Burlington, VT.

Background: The utility of routine histologic examination of lung specimens from patients with clinically primary spontaneous pneumothorax (SPTX) has been questioned in the surgical literature. However, in our experience, histologic examination of SPTX specimens yields findings that impact clinical management. We present the first detailed retrospective histopathologic assessment and cost analysis addressing whether routine histologic examination of clinically primary SPTX specimens is justified.

Design: Clinical and radiographic records of all patients undergoing surgical resection for SPTX at our institution from 2002-2012 and corresponding histologic slides were reviewed. Each specimen was assessed for histologic features typically associated with SPTX, as well as unexpected findings.

Results: Seventy-two patients considered clinically to have primary SPTX were identified, with 78 cases available for review. All cases demonstrated one or more findings typically associated with SPTX. Clinically significant unexpected histologic findings were identified in $7.7 \%$ of cases: 1 case of lung carcinoma, 1 case showing endometriotic stroma-lined cysts and adenomyosis on subsequent hysterectomy interpreted as pulmonary stromal endometriosis, 1 case with histologic findings compatible with Birt-Hogg-Dube syndrome (BHD) subsequently confirmed by genetic testing, and 3 cases consistent with "bong lung" with marijuana use confirmed on followup. With the exception of a cyst seen grossly in the case of stromal endometriosis, no distinctive gross findings were noted in cases with significant unexpected histologic findings.

Conclusions: Gross examination alone is inadequate for detecting unexpected clinically significant findings in lung specimens from patients with clinically primary SPTX. By contrast, routine histologic examination does identify unexpected findings that alter patient management and/or have important implications for relatives. In our series, these included a previously unrecognized case of BHD, which confers a substantial risk of renal carcinoma. According to our analysis, the cost of histologically examining all primary SPTX specimens received in a 10-year period is less than the average reported cost of treating one patient for advanced stage renal carcinoma. This is the first study to demonstrate that clinically primary SPTX specimens should be routinely examined histologically and is a cost-effective means of detecting unexpected findings with significant clinical impact.

2027 Tumor Infiltrating Lymphocytes Are Associated with Epithelial Expression of PD-L1 Protein, PD-L1 mRNA and Better Outcome in NonSmall Cell Lung Cancer

KA Schalper, VVelcheti, DE Carvajal-Hausdorf, VAnagnostou, K Syrigos, S Gettinger, $L$ Chen, R Herbst, DL Rimm. Yale School of Medicine, New Haven, CT; Cleveland Clinic, Cleveland, OH; Athens University School of Medicine, Athens, Greece.

Background: Although the presence of tumor infiltrating lymphocytes (TILs) has been associated with survival benefit in non-small cell lung cancer (NSCLC), the effect is significant but modest. In most instances the immune system generates only a partially effective anti-tumor response. Activation of the PD-1/PD-L1 axis induces tumor immune evasion and blockade of this pathway using immunostimulatory antibodies shows prominent anti-tumor responses. We determined the association between TILs, PD-L1 protein, PD-L1 mRNA and outcome in two retrospective NSCLC cohorts.

Design: The level of TILs, PD-L1 protein and in situ mRNA were measured in 544 stage I-IV NSCLC samples represented in two TMAs (Yale cohort [YC] $\mathrm{n}=204$; and Greek cohort $[\mathrm{GC}] \mathrm{n}=340$ ). The level of TILs was determined by pathologist evaluation of H\&E stained preparations using a 4-tier scale (0-3+). PD-L1 protein and mRNA were measured by quantitative fluorescence using the antibody clone $5 \mathrm{H} 1$ and the RNAscope paired-primer assay. UbC mRNA was used as positive control and DapB as negative control. Association of the variables with clinico-pathological characteristics and overall survival were determined.

Results: Elevated TILs (categories 2\&3+) were found in 34\% of cases from the YC and $38 \%$ of the GC. PD-L1 protein expression was detected in 36 and $25 \%$ of patients of the YC and GC, respectively. PD-L1 mRNA expression was found in $51 \%$ of cases from the $\mathrm{YC}$ and $53 \%$ of the GC. In both collections, PD-L1 protein and mRNA showed a positive non-linear relationship $\left(\mathrm{R}^{2}=0.182\right.$ in the $\mathrm{YC}$ and $\mathrm{R}^{2}=0.337$ in the $\left.\mathrm{GC}\right)$. In univariate analysis, the presence of increased TILs was positively associated with PDL1 protein and mRNA expression. Elevated TILs, PD-L1 protein or mRNA expression were independently associated with longer overall survival $(\log \operatorname{rank} \mathrm{P}=0.009$ in the 
$\mathrm{YC}$ and $\mathrm{P}=0.016$ in the GC for TILs; $\mathrm{P}=0.037$ in the $\mathrm{YC}$ and $\mathrm{P}=0.028$ in the $\mathrm{GC}$ for PD-L1 protein; and $\mathrm{P}=0.003$ in the $\mathrm{YC}$ and $\mathrm{P}=0.012$ in the $\mathrm{GC}$ for $\mathrm{PD}-\mathrm{L} 1 \mathrm{mRNA}$ ). Conclusions: The presence of increased TILs is observed in nearly $35 \%$ of NSCLC and is associated with PD-L1 expression and better outcome. Measurement of tumor PD-L1 protein and mRNA provide non-equivalent, complementary information. These assays could be used as companion biomarkers to predict response to anti PD-1/PDL1 therapies.

2028 Pulmonary Capillary Hemangiomatosis: A Clinicopathological Study of 45 Cases

A Seki, HR Green, TD Lee, R Saggar, MC Fishbein. UCLA, Los Angeles, CA.

Background: Pulmonary capillary hemangiomatosis $(\mathrm{PCH})$ is a proliferation of alveolar septal capillaries (Figure 1), and said to be a rare cause of pulmonary arterial hypertension (PAH). Pulmonary veno-occlusive disease (PVOD) (Figure 2) is clinically similar to $\mathrm{PCH}$; the two entries are said to often coexist.
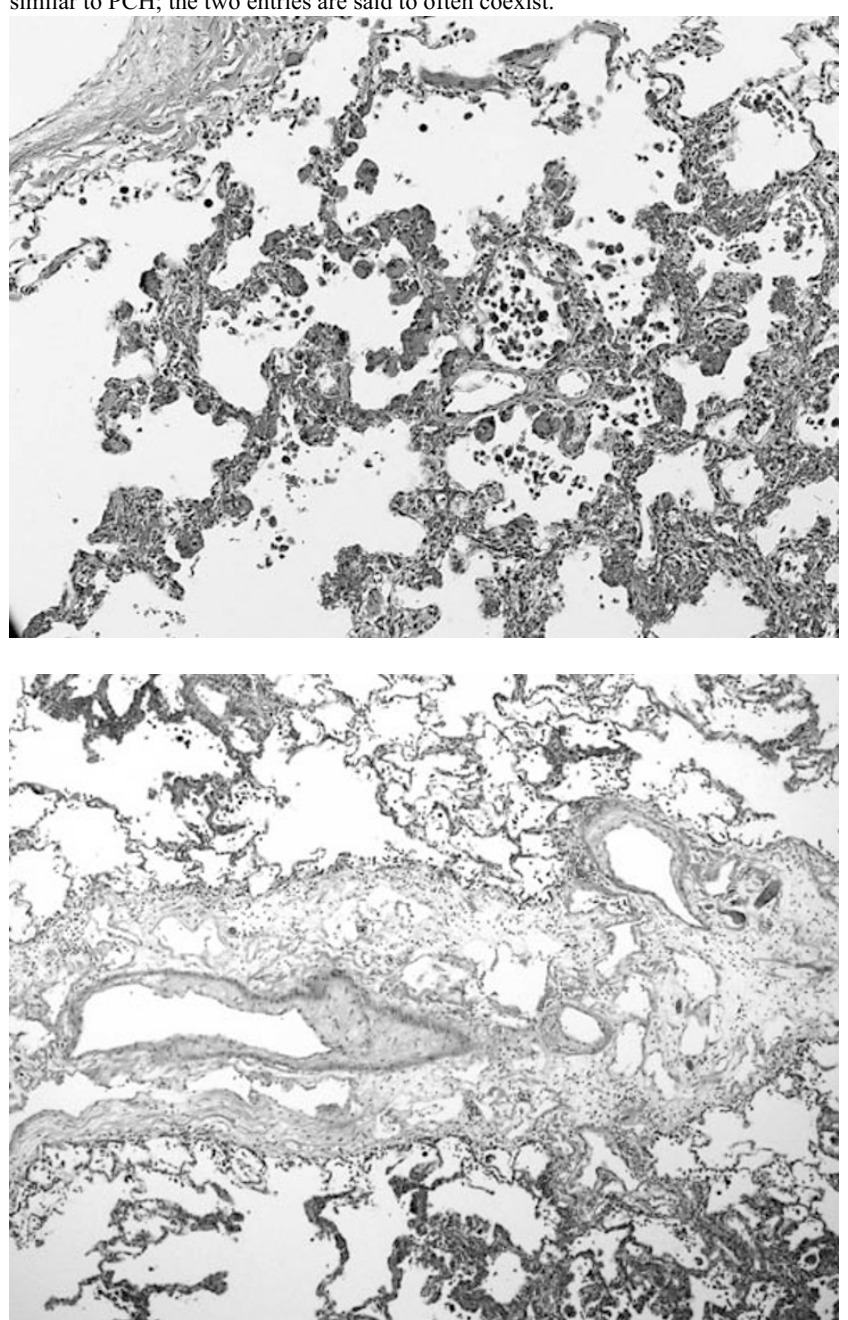

Design: Pathology records from 1997-2013 were reviewed. There were 45 cases with $\mathrm{PCH}, 20$ autopsies and 25 lungs explanted at transplantation. Standard sections of lungs were reviewed. The major patterns of parenchymal and vascular disease and percentage of tissue with $\mathrm{PCH}$, and other abnormalities were documented.

Results: The mean age of the pts was 54 yrs with a wide range from 17-93. PCH occurred with equal frequency in females, $\mathrm{n}=23$, and males, $\mathrm{n}=22$. Collagen vascular disease was seen in $57.8 \%(\mathrm{n}=26)$ of pts with PCH. Systemic or limited sclerosis was diagnosed in 21 pts. The diagnosis of PCH or PVOD was made clinically in only two cases. Clinically, PAH was identified in $86.7 \%(\mathrm{n}=40)(\mathrm{mPAP}>25 \mathrm{mmHg})$. The most common symptom was shortness of breath. Chest $\mathrm{CT}$ in $42 \mathrm{pts}$ showed ground grass opacity in $57.1 \%(\mathrm{n}=24)$. The involvement of lung tissue with $\mathrm{PCH}$ varied from 3.6$90.0 \%$ (mean 34.5 ). PVOD was present in $43.0 \%$ of case $(n=18)$. Over $50 \%$ of pts had interstitial lung disease: usual interstitial pneumonia (UIP) in $32.0 \%(\mathrm{n}=17)$, nonspecific interstitial pneumonia (NSIP) in 4.4\% ( $\mathrm{n}=2)$, and UIP\&NISP in $20.4 \%(\mathrm{n}=13)$. Arterial changes of PAH were seen in $93.3 \%(\mathrm{n}=42)$; Grade I-II of Heath and Edwards, $48.9 \%$ $(\mathrm{n}=22)$; Grade III and IV, $44.4 \%(\mathrm{n}=20)$. No $\mathrm{pt}$ had hemoptysis, yet hemosiderin-laden macrophages a marker of pulmonary hemorrhage was present in $51.1 \%(\mathrm{n}=23)$.

Conclusions: $\mathrm{PCH}$ in the lung is not as rare as reported, and is strongly associated with $\mathrm{PAH}$ and interstitial lung disease, especially scleroderma. PVOD in pts with $\mathrm{PCH}$ is common $(43 \%)$ but not invariably present.
2029 Expression of CD30 Antigen in Human Malignant Mesotheliomas:

\section{A Potential Therapeutic Target}

A Sethi, K Sheibani, MA Vasef. University of New Mexico, Albuquerque, NM; Western Medical Center, Santa Ana, CA.

Background: Malignant mesothelioma (MM) is an aggressive neoplasm with poor response to surgery, chemotherapy, and radiation. Prior studies have failed to identify a targetable genetic abnormality such as BRAF or activating EGFR mutations, or HER2 gene amplifications. Recently, Brentuximab, an FDA-approved anti-CD30 targeted therapy (Brentuximab) has shown improved outcome in patients with Hodgkin lymphoma and anaplastic large cell lymphoma. With availability of Brentuximab, and given the poor response of MM to conventional treatment, determination of CD30 expression status in MM is important to assess as a potential beneficial targeted therapy. Design: Paraffin embedded diagnostic tissue blocks from 20 patients with history of asbestos exposure and immunohistochemically proven pleural MM were selected for this study. A tissue microarray (TMA) was constructed by arranging 4 cores from each selected tissue in seven rows using manually operated tissue puncher. $0.6 \mathrm{~mm}$ in diameter tissue cylinder removed from donor blocks and oriented precisely within the recipient block. Immunohistochemistry was performed using CD30 antibody (DAKO) on $4 \mu \mathrm{m}$-thick microarrays tissue sections.

Results: Six out of 20 (30\%) MM expressed CD30 including 3 epithelioid, 2 sarcomatoid, and 1 biphasic type. The percentage of CD30-positivity ranged from 30 to over $80 \%$ of tumor cells in $\mathrm{CD} 30+\mathrm{MM}$ cases and the intensity ranged from weak $(1+)$ to bright $(3+)$
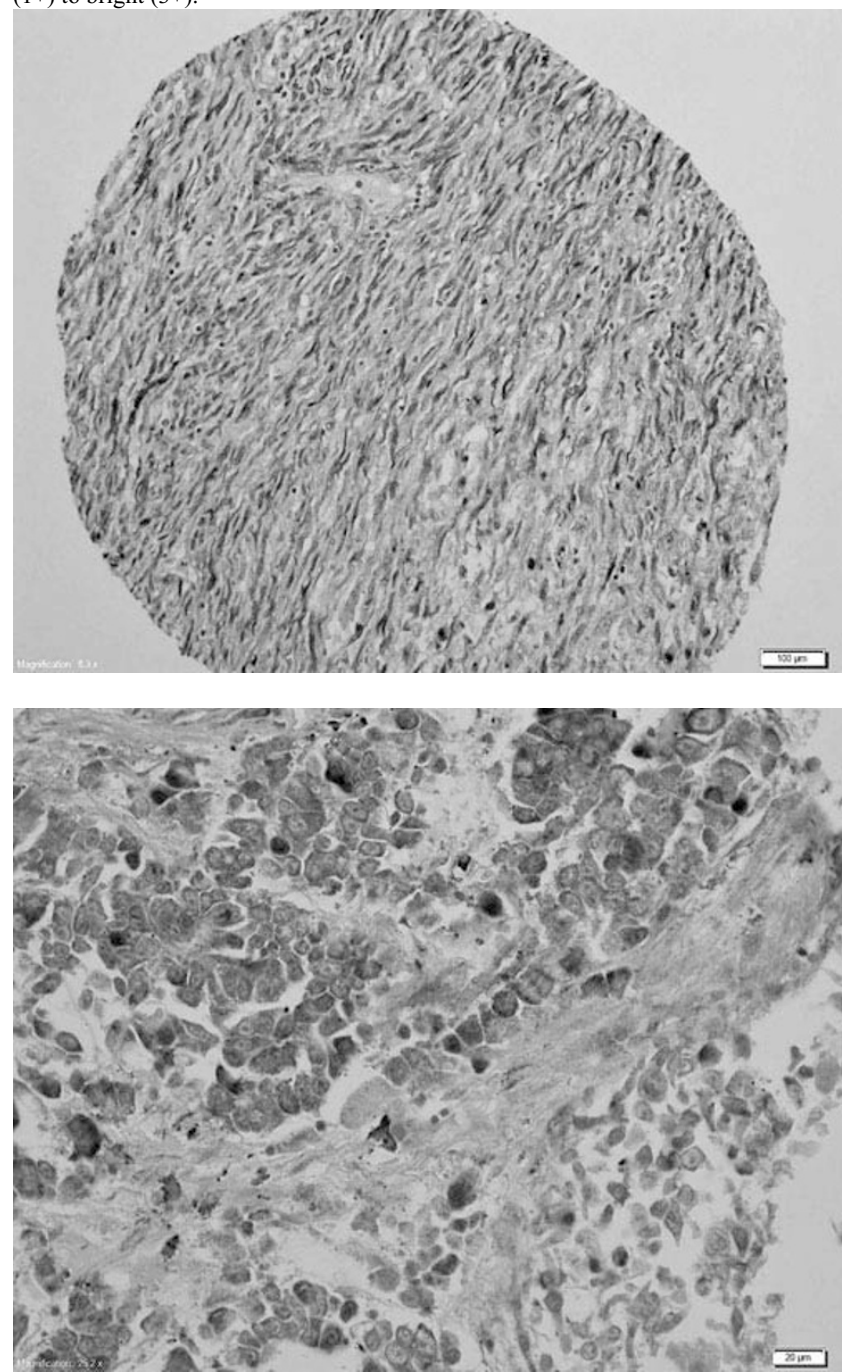

Conclusions: Our study shows that CD30 is expressed in $30 \%$ of MM cases. Given the availability of a monoclonal anti-CD30 antibody (Brentuximab) which is currently approved for treatment of Hodgkin and anaplastic large cell lymphoma, our findings suggest that patients with CD30 expressing MM may benefit from administration of Brentuximab. The potential benefits needs to be further assessed and validation by enrolment of patients with CD30 expressing MM in clinical trials. 
2030 Expression Patterns of FABP5 Are Associated with Grade of Pulmonary Neuroendocrine Tumors

SM Smith, CA Powell, MW Nasser, J Fukuoka, J Jen, TJ Franks, WD Travis, RK Ganju, $K$ Shilo. The Ohio State University, Columbus, OH; Nagasaki University, Nagasaki, Japan; Mayo Clinic, Rochester, MN; Joint Pathology Center, Silver Springs, MD; Memorial Sloan-Kettering Cancer Center, New York, NY.

Background: The transport of fatty acids within the intracellular compartments is dependent on a family of 14-15 kDa proteins referred to as fatty acid binding proteins (FABPs). Epidermal FABP, also known as FABP5, is expressed most abundantly in epidermal cells and also in adipocytes, macrophages and dendritic cells. FABP5 has been found to be over-expressed bladder, breast and prostate carcinomas. Whether FABP5 has a role in tumorigenesis of lung neuroendocrine tumors (LNET) is unknown. This study seeks to delineate FABP5 expression patterns in LNET and potential correlation with clinicopathologic findings.

Design: Tissue microarray based samples from 184 patients with LNET were evaluated for FABP5 expression with a polyclonal goat anti-human antibody $(1: 100, \mathrm{R} \& \mathrm{D}$ Systems, Minneapolis, MN). FABP5 expression was assessed in comparison to normal lung parenchyma as cytoplasmic (negative, low and high) as well as dendritic (nuclear/ cytoplasmic) staining ( $<50 \%$ cells, low), $(\geq 50 \%$ cells, high) and negative. The expression patterns were correlated with clinicopathologic variables (SYSTAT, Chicago, IL).

Results: Fifteen of 35 (42.9\%) typical carcinoid tumors (TC), 12 of 27 (44.4\%) atypical carcinoid tumors (AC), 23 of 42 (54.8\%) small cell lung carcinomas (SCLC) and 7 of $19(36.8 \%)$ large cell neuroendocrine carcinomas (LCNEC) showed high cytoplasmic FABP5. Dendritic pattern of FABP5 expression was seen in 28 of $35(80.0 \%)$ TC, 18 of 27 (66.7\%) of AC, 37 of 42 (88.1\%) SCLC, and 17 of 19 (89.5\%) LCNEC. Both cytoplasmic and dendritic patterns of FABP5 expression were associated with tumor grade (high-grade vs. low-grade LNET) and size $(<2 \mathrm{~cm}$ vs. larger LNET) [p $<0.05]$, but not with patients' age, gender, survival and tumor stage.

Conclusions: Cytoplasmic expression of FABP5 is seen in significant proportion of LNET suggesting its role in their tumorigenesis and association with a more aggressive phenotype. The presence of frequent FABP5-expressing intratumoral dendritic cells warrants further investigation of their role in cell-mediated immunity and development of immunological tolerance in lung tumors.

2031 EGFR Mutation Specific Antibodies Can Detect the Majority of EGFR TKI Sensitizing Mutations in Adenocarcinoma of the Lung

$C C$ Solomides, $R$ Draganova-Tacheva, $M$ Bibbo, $S$ Ren, $Y$ Shi, IE Palazzo, $R$ Birbe, $R$ $O$ 'Neill, SC Peiper, Z-X Wang. Jefferson Medical College, Philadelphia, PA; Jeanes Hospital, TUHS, Philadelphia, PA.

Background: Activating mutations in the tyrosine kinase domain of the EGFR gene that lead to tumor growth and progression have been detected in lung adenocarcinoma. Consequently, EGFR has become a target for anti-cancer drug therapy. The most common mutations associated with sensitivity to EGFR TKIs include exon 19 deletions and the L858R point mutation. These mutations are currently detected by Sanger sequencing in our Molecular Lab. Small biopsies are frequently used for lung cancer diagnosis resulting often in specimens with low tumor content. Microdissection is performed for most specimens to reach $30 \%$ tumor cells to meet the detection sensitivity of sequence based assays. In this study we examine the utilization of new anti-EGFR L858R (SP125) and anti-EGFR E746-A750 del (SP111) rabbit primary monoclonal antibodies in FFPE tissues.

Design: Thirty one (31) cases of lung adenocarcinoma with adequate FFPE tissue previously tested for EGFR mutations in our molecular lab were selected. Ten (10) cases were wild type, nine (9) cases were positive for the EGFR L858R mutation and twelve (12) were positive for the EGFR exon 19 deletions ( 9 had del E746-A750 and 3 contained other deletions). All cases were stained with both anti-EGFR L858R and anti EGFR E746-A750 del antibodies (Ventana Medical Systems, Inc, Tucson AZ), using the Ventana BenchMark Ultra immunohistochemical (IHC) stainer platform. Moderateto-strong staining by IHC was considered positive result; absent or equivocal staining was considered negative. The results were obtained by consensus of four pathologists. Results: All cases ( $\mathrm{n}=9: 100 \%$ ) that were positive for the EGFR L858R mutation by sequencing were also positive by IHC (anti-EGFR L858R antibody); none of these showed positivity for the anti EGFR E746-A750 del antibody. The nine (9) of twelve (12) cases that were positive for the EGFR exon 19 deletion E746-A750 by sequencing were also positive by IHC (anti EGFR E746-A750 del antibody); the (3) specimens with other exon 19 deletions were negative for this antibody. None of these 12 showed positivity for the anti-EGFR L858R antibody. All ten cases that were wild type by sequencing were negative by IHC for both antibodies.

Conclusions: Immunohistochemistry on FFPE tissues with monoclonal antibodies specific for L858R and the most common form of exon 19 deletion may be of clinical efficacy in the initial evaluation of lung adenocarcinomas and serve as a guide to further molecular analysis.

2032 Epithelial-Mesenchymal Plasticity in Malignant Pleural Mesothelioma: The Stem Cell Factors Sox10 and Nestin Are Associated with Its Sarcomatoid End

A Soltermann, S Thies, L Frischknecht, I Opitz, E Felley-Bosco, W Weder, H Moch. University Hospital Zurich, Zurich, Switzerland.

Background: Malignant pleural mesothelioma (MPM) with its epithelioid and sarcomatoid pattern allows for studying intrinsic epithelial-mesenchymal (EMT) transition. Cells undergoing EMT may display stem cell (SC) traits. We hypothesized that the neural crest stem cell markers Sox 10 (transcription factor Sry-box 10) and nestin (intermediate filament protein) may be related to the "M-end", thus to sarcomatoid MPM and to the EMT marker periostin (extracellular matrix). Sox10 regulates the nestin neural enhancer in melanoma.

Design: Tumor tissue of 192 consecutive MPM patients was analysed by IHC on tissue microarrays. 111 patients underwent trimodal therapy including neo-adjuvant chemotherapy (CTX), either cisplatin/gemcitabine or cisplatin/pemetrexed, following extrapleural-pleuropneumonectomy (EPP) and optional adjuvant radiotherapy. Pre-CTX biopsies were available for 88/111 EPP patients. 81 patients had either pleurectomy/ decortication (P/D) or no surgery performed. Immunoreactivity was H-scored (intensity multiplied by frequency) on 2 cores (biopsy) or 4 cores (surgical specimen), respectively, for a total of 926 cores.

Results: The histologic MPM pattern was epithelioid in $112(58 \%)$, biphasic in 69 $(36 \%)$ and sarcomatoid in $11(6 \%)$. High protein expression of nuclear Sox 10 as well as cytosolic nestin and periostin in tumor cells increased towards the sarcomatoid pattern, the 3 proteins also being positively correlated among each other. In particular, nestin distinguished between epithelioid and biphasic but not between biphasic and sarcomatoid in both pre- and post-CTX MPM. Sox10 distinguished between biphasic and sarcomatoid in pre-CTX but between epithelioid and biphasic in post-CTX. Cis/ Gem but not Cis/Pem CTX increased Sox 10 and periostin. Sox 10 also increased in 5 MPM diagnosed as epithelioid on biopsy but biphasic on surgical specimen. Sarcomatoid pattern and high nestin correlated with poor prognosis in all patients. In the EPP subset, high nestin (median overall survival 21.9 versus 25.5 months), high pT, high pN and lung infiltration were adversely prognostic next to histology (all p-values $<0.05$ ).

Conclusions: Next to the loss of diagnostic markers calretinin and D2-40, the sarcomatoid "M" end of MPM is characterized by an increase of stem cell traits. Protein expression of Sox 10 and nestin is further modulated by chemotherapy.

\section{PD-L1 Expression in Stage IV Lung Cancer}

Y Sun, $H$ Ye, $H W u$, C O'Hara. Boston Medical Center, Boston, MA; Beth Israel Deaconess Medical Center, Boston, MA; Children's Hospital, Boston, MA

Background: Tumors have developed multiple mechanisms to shut down the immune pathway for surveillance against cancer. The programmed death-1/programmed death ligand-1 (PD-1/PD-L1) pathway has emerged as an important tumor evasion mechanism. Two recent clinical trials highlighted the therapeutic potential of an agent that targets PD-1/PD-L1 in patients with advanced non-small cell lung cancer (NSCLC). However, the challenge has been the identification of predictive biomarkers that could be used to identify potential candidates for such treatment. In the current study, we use immunohistochemistry (IHC) to evaluate PD-L1 expression in stage IV lung cancer. Design: We retrospectively reviewed 24 patients with stage IV lung squamous cell carcinoma or adenocarcinoma. Sections of formalin-fixed tumor specimens were subjected to IHC to evaluate the patterns of PD-L1 expression.

Results: The tumor specimens included 14 adenocarninomas, six squamous cell carcinoma and four NSCLC. The expression of PD-L1 was demonstrated in the cell membrane, cytoplasm, or both (clone 5H1).

Fig. 1 PD-L1 expression in stage IV lung cancer

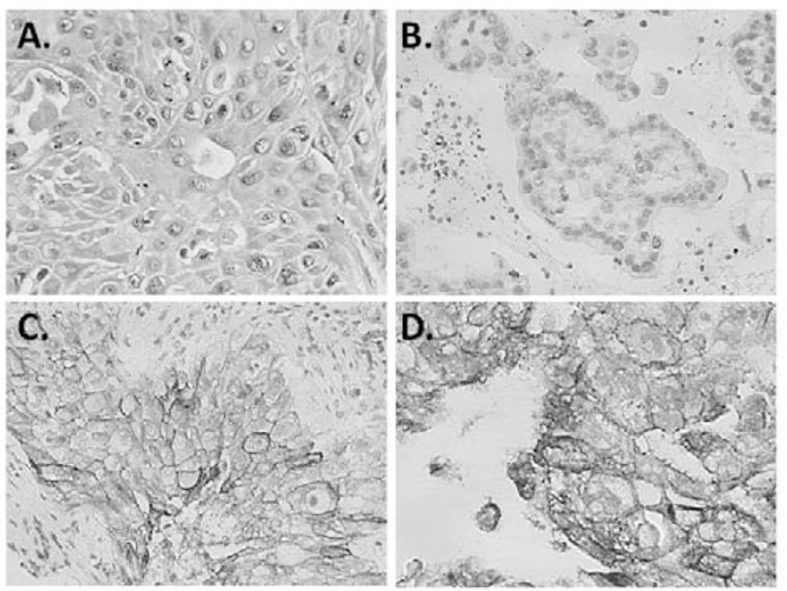

A. H\&E; B. Adenocareinoma; C. Squamous cell eareinoma (low power); D. Squamous cell eareinoms (high power)

Interestingly, $67 \%$ of the stage IV lung squamous cell carcinoma expressed high level of PD-L1. Of the stage IV NSCLC, 25\% expressed PD-L1, (Table 1) whereas none of the stage IV adenocarcinoma expressed PD-L1.

Table I. PD-L1 expression in stage IV lung squamous cell carcinoma (SCC) and NSCLC

\begin{tabular}{|c|c|c|c|c|c|}
\hline Case & Gender & Age & Diagnosis & Survival (yrs) & PD-L1 \\
\hline 1 & $\mathbf{M}$ & 61 & Mod diff SCC & 0.2 & pos \\
\hline 2 & $\mathbf{M}$ & 72 & Mod diff SCC & 4.1 (alive) & pos \\
\hline 3 & $\mathbf{M}$ & 73 & SCC & 0.1 & pos \\
\hline 4 & $\mathbf{F}$ & 49 & SCC & - & pos \\
\hline 5 & $\mathbf{M}$ & 61 & Mod diff SCC & 0.9 & neg \\
\hline 6 & $\mathbf{F}$ & 71 & Poorly diff SCC & 0.5 & neg \\
\hline & & & & & \\
\hline 7 & $\mathbf{F}$ & 61 & Poorly diff NSCLC & 1.5 & neg \\
\hline 8 & $\mathbf{F}$ & 67 & Poorly diff NSCLC & 0.2 & neg \\
\hline 9 & $\mathbf{F}$ & 59 & Poorly diff NSCLC & 0.4 & neg \\
\hline 10 & $\mathbf{M}$ & 59 & Poorly diff NSCLC & 0.3 & pos \\
\hline
\end{tabular}


Table II. PD-L1 expression in stage IV lung adenocarcinoma (Ad)

\begin{tabular}{|l|l|l||l||l||}
\hline Case Number & Gender & Median Age & Diagnosis & $\begin{array}{l}\text { Median Survival } \\
\text { (yrs) }\end{array}$ \\
\hline $\mathbf{n = 1 0}$ & $\mathbf{F}$ & $\mathbf{6 5}$ & Mod-poorly diff Ad & $\mathbf{0 . 6}$ \\
\hline $\mathbf{n = 4}$ & M & $\mathbf{5 6}$ & Mod-poorly diff Ad & $\mathbf{0 . 4}$ \\
\hline
\end{tabular}

Conclusions: Our results suggest that the expression of PD-L1 on stage IV lung cancer is predominantly on squamous cell carcinoma. Further study is ongoing to evaluate PDL1 expression in stage IV lung cancer obtained from a large cohort of patients $(n=80)$.

2034 ALK Immunohistochemical Staining with Anti-ALK Antibody (Clone D5F3) Is a Reliable Method to Detect ALK Rearrangement in Lung Adenocarcinomas: A Study of Consecutive 879 Cases in The Chinese Population

$Y$ Sun, L Zhou, X Huang, Q Kang, L Jia, X Li, D Cao. Peking University Cancer Hospital, Beijing, China.

Background: Recent studies have shown that patients whose lung adenocarcinomas harbor anaplastic lymphoma kinase $(A L K)$ gene rearrangement benefit from ALK tyrosine kinase inhibitor (crizotinib) treatment. Detection of $A L K$ rearrangement by fluorescence in situ hybridization (FISH) is considered to be the standard procedure. However, FISH testing is costly, labor intensive and requires expertise, and for this matter it may be not be available in some laboratories. Using immunohistochemistry (IHC) with an anti-ALK antibody is a potential alternate method to detect $A L K$ rearrangement. Here we evaluated the feasibility of using ALK IHC as a reliable method of detecting $A L K$ rearrangement in a large series of lung adenocarcinomas.

Design: A consecutive series of 879 surgically resected pulmonary adenocarcinomas between 2008 and 2012 were included. Tissue microarrays (TMAs) were constructed from formalin fixed paraffin embedded tissue blocks (1-mm core, 2 cores per case) and were immunohistochemically stained with a rabbit monoclonal antibody to ALK protein (Ventana prediluted, Clone D5F3). To delineate ALK IHC staining pattern, we also stained one tissue block from all ALK IHC-positive cases on TMAs. A case with any tumor cell staining was considered ALK IHC-positive. ALK IHC-positive cases and representative IHC-negative cases were further tested for $A L K$ arrangement using a dual-color break-apart probe specific to $A L K$ locus (Vysis LSI ALK Break Apart FISH Probe, Abott Molecular, USA). More than $15 \%$ tumor cells showing split signals was considered positive for $A L K$ rearrangement.

Results: Among 879 cases tested, 40 (4.6\%) were ALK positive by IHC. The ALK staining was strong and diffuse (nearly $100 \%$ cells) in 39/40 cases on both TMAs and tissue blocks. In the remaining case, ALK staining was diffuse and strong on TMA but ont the tissue block the staining was heterogeneously positive in $60 \%$ cells in both intensity and distribution. All 40 ALK IHC-positive tumors were positive for $A L K$ rearrangement by FISH study. All randomly selected 209 ALK IHC-negative tumors were FISH negative for $A L K$ rearrangement. The sensitivity and specificity of using IHC with Ventana anti-ALK antibody (clone D5F3) to detect $A L K$ rearrangement in our series was $100 \%$ and $100 \%$, respectively.

Conclusions: ALK rearrangement is a relatively uncommon event $(4.6 \%)$ in lung adenocarcinoams in the Chinese population. IHC using a specific antibody to ALK protein (Ventana D5F3) is a sensitive and reliable method to detect $A L K$ rearrangement.

2035 Blastomatoid Carcinosarcoma of the Lung Is a Biphasic Tumor of High-Grade Malignancy That Needs to Be Distinguished from Pulmonary Blastoma: A Clinicopathological and Immunohistochemical Analysis of 13 Cases

M Suzuki, S Ota, Y Inayama, T Yokoi, K Kawachi, K Wakasa, T Iijima, D Mori, Y Tsukamoto, S Yokoyama, T Takemura, Y Honda, R Haba, O Matsubara, EJ Mark, Y Nakatani. Graduate School of Medicine, Chiba University, Chiba, Japan; Chiba University Hospital, Chiba, Japan; Massachusetts General Hospital and Harvard Medical School, Boston, MA.

Background: Pulmonary blastoma (PB) and carcinosarcoma (CS) often pose a difficulty in differential diagnosis. Blastomatoid carcinosarcoma (BCS) is a variant of pulmonary $\mathrm{CS}$ that shows high-grade fetal adenocarcinoma (H-FLAC) as the epithelial component, and has long been confused with PB (Nakatani Y et al. Am J Surg Pathol, 2004). PB frequently harbors mutation of the $\beta$-catenin gene, whereas BCS lacks the mutation. This indicates a different histogenesis of the two tumors. Only a few cases of BCS, however, were reported and its clinicopathological features are yet to be clearly defined. Design: Thirteen cases of BCS were retrieved from the surgical and autopsy files of our departments. We analyzed clinical and pathological findings. We used a panel of antibodies including $\beta$-catenin, $\alpha$-fetoprotein (AFP), thyroid transcription factor- 1 (TTF-1), synaptophysin, chromogranin A, desmin and myogenin.

Results: BCS occurred predominantly in men (12 men, 1 woman). Mean age was 64.9 years (21-87). Nine of 10 patients were heavy smokers. Mean tumor size was $6.4 \mathrm{~cm}(1.8-$ 12.5). TNM staging classified 1 patient as stage I, 5 patients as stage II, and 6 patients as stage 3 or 4 . Nine of 12 patients died (follow-up periods of 1-108 months, average 21.3 months). Histolopathogically, BCSs had H-FLAC as its predominant epithelial component, which formed complex glandular structures lined by glycogen-rich columnar cells resembling endometrioid adenocarcinoma. None of the BCSs displayed morules. The neoplastic mesenchyme in BCSs was composed of oval to spindle cells with various types of differentiation. Rhabdomyosarcomatous and/or chondrosarcomatous differentiation was focally seen in all cases. BCS showed focal differentiation of squamous cell carcinoma in 3 , conventional adenocarcinoma in 1 , small cell carcinoma in 1 , and large cell neuroendocrine carcinoma in 1 case. Immunohistochemically, all BCSs had membranous localization of $\beta$-catenin in the epithelial component. Neoplastic epithelial cells stained for AFP in 100\% (6/6), for chromogranin A and/or synaptophysin in $100 \%(6 / 6)$, and for TTF-1 in $22 \%(2 / 9)$ of cases.
Conclusions: BCS differs from PB in several important ways, including epidemiology, staining patterns and prognosis.

2036 Pleuroparenchymal Fibroelastosis and Non-Specific Interstitial Pneumonia in Pulmonary Graft-Versus-Host-Disease Following Hematopoietic Stem Cell Transplantation

$Y$ Takeuchi, A Miyagawa-Hayashino, F Chen, H Date, H Haga. Kyoto University Hospital, Kyoto, Japan.

Background: Pulmonary manifestations reported as chronic graft-versus-host-disease (cGVHD) after hematopoietic stem cell transplantation (HSCT) include constrictive bronchiolitis obliterans (CBO), lymphocytic bronchiolitis (LB), and veno-occlusive disease (VOD). Recently, pleuroparenchymal fibroelastosis (PPFE) or non-specific interstitial pneumonia (NSIP) have been described in bone marrow transplant recipients, but the histological features of pulmonary disease after HSCT have not been described systematically.

Design: Retrospective review of 21 patients, who underwent lung transplantation or lung biopsy for pulmonary disease after HSCT between April 2004 and June 2013, was conducted.

Results: The patient age at lung transplantation or at the time of biopsy ranged from 8 to 57 years (median, 26 years). Eleven were male and 10 female. Primary disease for HSCT was hematological disease including leukemia or myelodysplastic syndrome in 19 patients, and neuroblastoma in 2 . Sixteen patients had cGVHD in other organs other than lung with 10 involving skin, 6 involving liver, and 6 involving lacrimal or salivary gland. Bilateral lung transplantation was performed in 15 , unilateral lung transplantation in 5 and wedge biopsy in 1. Lung transplantation or biopsy for pulmonary disease after HSCT was performed at a median of 4.3 years (range, 0.9- 14.8 years). Histologically, all 21 cases showed CBO. Concurrent features of CBO included LB in 10 and VOD in 3. We identified PPFE in 16 patients (76\%); subpleural distribution was seen in 16 patients, paraseptal distribution in 11, and centrilobular distribution in 14. NSIP pattern was identified in 16 patients $(76 \%)$; 10 had fibrotic NSIP and 6 had cellular NSIP. NSIP was seen focally in upper lobe in 7 and lower lobe in 13 with one case showing diffusely. Conclusions: PPFE and NSIP were frequently seen in HSCT patients in addition to $\mathrm{CBO}$. The possible causes may include drugs, radiation, or cGVHD. Most patients had cGVHD in other organs concurrently, PPFE and NSIP may represent the manifestation of pulmonary cGVHD.

2037 Revisiting Histological Classification of Congenital Cystic Lung Disease Based on Etiology and Fetal Diagnosis

M Tanaka, R Ijiri, M Yoshida, M Yoshida, K Nozawa, N Aida, M Shinkai, M Kawataki, MOhyama, Y Itani, Y Tanaka. Kanagawa Children's Medical Center, Yokohama, Japan. Background: Congenital cystic lung disease (CCLD) is one of common anomalies seen in neonates. Congenital cystic adenomatoid malformation/congenital pulmonary airway malformation (CCAM/CPAM), one of the major CCLD, is now generally diagnosed according to the classification by Stocker. Recently, newly developed imaging devices and techniques made prenatal diagnosis of CCLD possible in some cases. Many cases of bronchial atresia (BA) or intralobar/extralobar sequestration (ILS/ELS) have been diagnosed prenatally with appropriate devices. Also several new entities of CCLD such as fetal lung interstitial tumor have been recognized. In some cases, diagnosis of these lesions can be confusing because of the overlap in histological findings. The cases with fetal diagnosis often have sufficient clinical information and radiological studies, and are usually suitable for histological examination because of minimal inflammatory changes. In order to clarify histological classification, surgical specimens of CCLD with fetal diagnosis were investigated and reported in this study.

Design: Thirty-eight CCLD cases with fetal diagnosis were submitted for histological examination in our institute; 8 cases were excluded because of poor histological condition due to extensive inflammatory changes and 30 cases were used for the study. Two autopsy cases were included. Histological and radiological reviews were performed in selected cases.

Results: The cases were categorized into 5 groups: group A (cystic lesion with linings containing mucus-producing cells) 13 cases, group B (cystic lesion associated with airway obstruction) 7 cases, group C (ILS/ELS) 7 cases, group D (small cystic lesion mimicking incompletely developed fetal lung) 1 case, and group E (others) 2 cases. Group A included a case with only small cystic lesions and clusters of mucus-producing cells. Group B consisted of 6 cases with cystic lesions with adenomatoid appearance and a case of lobar emphysema. Four cases originally diagnosed as Stocker type 2 CCAM were re-categorized to BA (group B) by reviewing the radiological examination. Conclusions: Majority of CCLD was classified into group A, B, and C. Clear histological classification was possible by excluding severely inflamed cases. Classification of CCLD based on etiology and reflecting radiological and histological findings is desirable.

2038 Cancer Stem Cells Expression in Non Small Cell Lung Cancer

PA Toro, C Galvez, S Ciguenza, E Rojas, E Cecilia, E Andrada, I Aranda. Hospital General Universitario de Elche, Elche, Spain; Hospital General Universitario de Alicante, Alicante, Spain.

Background: To date, there is no single immunohystochemistry (IHC) marker that can be used routinely for prognostic purposes in early stage Non Small Cell Lung Cancer (NSCLC). Over the past years, Cancer Stem Cells (CSC) expression has been a focus of attention due to its potential role as independent prognostic tumour markers in lung tumors otherwise similar. We selected a group of CSC markers that have already been shown to participate in the genesis of NSCLC. We observed their IHC expression in a well described cohort of stage I and II NSCLC patients without neoadjuvant chemotherapy, in order to assess its prognostic value. 
Design: - A total of 90 cases of NSCLC with complete follow-up were retrieved from the Surgical Pathology files: 60 adenocarcinomas (AC) and 30 squamous cell carcinomas (SCC).

- Using tissue microarrays specimens, we investigated the expression of CD24, CD44, D2-40, ALDH-1 and SOX-2.

- The log-rank (Mantel-Cox) test and Kaplan-Meier estimation were used to estimate the probability of overall survival (OS) and disease-free survival (DFS).

Results: - Patients age ranged from 47 to 85 years; 70 men and 20 women.

- SOX-2, CD44, ALDH1 and D2-40 overexpression was predominantly seen in SCC whereas expression of CD24 was most common in $\mathrm{AC}(\mathrm{p}<0.05)$

- Overall, there was a statistically significant relationship between low expression of CD24 and better OS (cut-off value of 50; $\mathrm{p}=0.02$ ). By histologic subtype, similar results were observed in AC (CD24 overexpression related with lower DFS and OS; $\mathrm{p}=0.01$ and 0.06 )

- Globally, CD44 expression was more commonly associated with improved OS $(\mathrm{p}=0.08)$. In a subgroup analysis, a significant association was evident in AC (increased OS and DFS, $\mathrm{p}=0.045$ and 0.12 ; cut-off values of 5)

- SOX-2 overexpression in AC was related to an improvement in both OS and DFS $(\mathrm{p}=0.18$ and 0.07 ; cut off value 50 ) Other markers showed no relation with survival. Conclusions: Our findings suggest that overexpression of CD24 is a significant predictor of poor outcomes in AC. Although it did not reach statistical significance, the relationship observed in AC between SOX-2 and CD44 overexpression and increased survival is interesting, as it suggests their potential use as prognostic markers. There are conflicting results in the literature regarding the expression of CSC in lung tumors. Our results provide new insights in the pathways involved that could be relevant for the selection of patients' treatments.

2039 Peilobular Margination by Elastosis: A Robust Histologic Marker of Usual Interstitial Pneumonia

T Ushigusa, T Tanaka, K Tsuruno, N Tawada, K Tabata, Y Kashima, K Kataoka, Y Kondoh, H Taniguchi, J Fukuoka. Nagasaki University Graduate School of Biomedical Sciences, Nagasaki, Japan; Toyama University Hospital, Toyama, Japan; Tosei General Hospital, Seto, Aichi, Japan.

Background: Accurate distinction of histological usual interstitial pneumonia (UIP) from other patterns, especially non-specific interstitial pneumonia (NSIP), is often challenging for pathologists. We routinely use Elastica van Gieson (EVG) staining to highlight elastic fibers for lung cases and have noticed that perilobular accumulation of elastic fiber is a frequent finding in UIP. We investigated the usefulness of this elastic fiber accumulation in UIP and NSIP cases.

Design: We retrospectively selected surgical lung biopsy from 87 consecutive chronic interstitial pneumonia cases under the diagnosis of either UIP or NSIP. All cases had biopsied samples from segment 5 and 9 . The amount of elastic fiber accumulation in the margin of lobules (Perilobular margination by elastosis: PLME) was semiquantitatively graded with EVG staining: 0 , none; 1 , mild; 2 , moderate; 3 , severe by 2 pathology trainees, and among in several lobules inside the biopsy, 5 highest scores of PLME were obtained for each biopsy (Fig.1). Average and highest PLME score for each biopsy were calculated by the obtained scores. All data were acknowledged as reasonable by senior pathologists.

Figure 1: Perilobular Margination by Elastosis (PLME), 2 lobules showing score 2 .

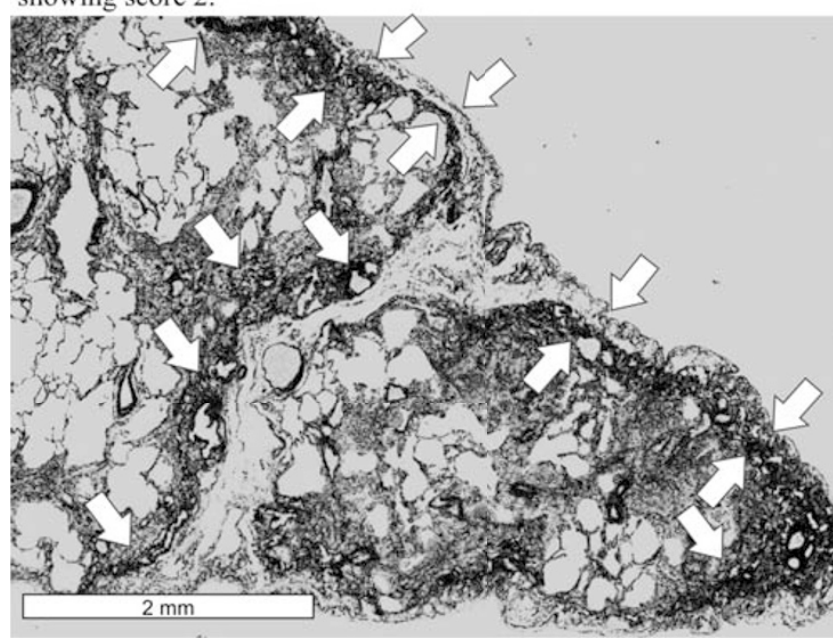

Results: The kappa agreement of the 2 observers was 0.63 (S5) and 0.60 (S9). PLME score was positively correlated with UIP histology in both S5 and S9 for both average and highest scores (all p values $<0.0001$ ). Among them, the receiver operating characteristic (ROC) curve provided that S9 average PLME score, cutoff of 1.6, and S9 highest PLME score, cutoff score of 2.0, as the best (Fig.2). When highest S9 PLME score was applied, the specificity was $100 \%$.
Figure 2: The ROC curve of S9 highest PLME score (UIP positive, NSIP negative).

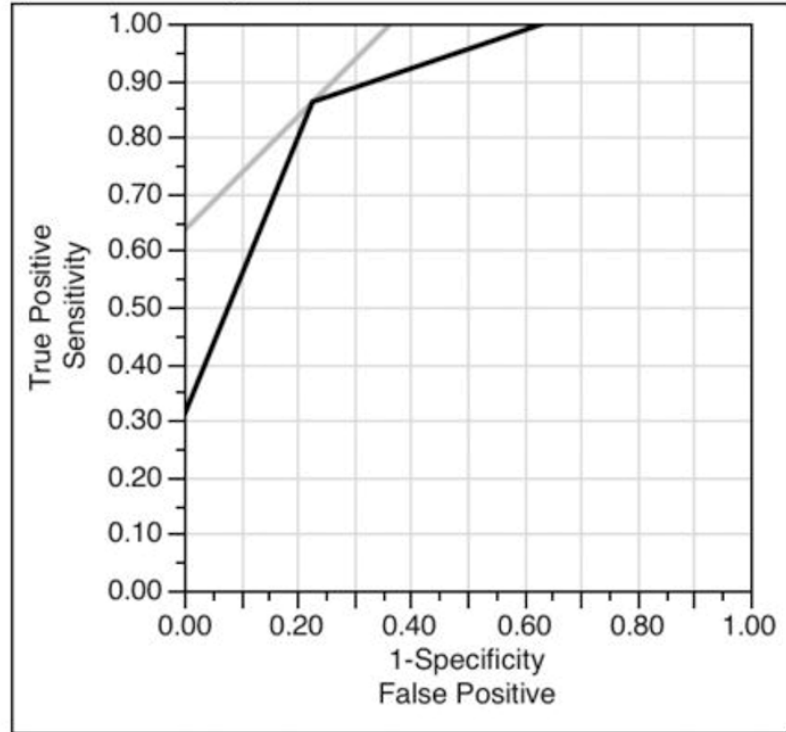

Conclusions: PLME identified by EVG staining was a distinctive, easy, and reproducible marker for UIP. More than moderate level of PLME in lung base can be strong negative indicator of NSIP.

2040 Clinicopathologic Features Associated with Anaplastic Lymphoma Kinase (ALK) Gene Rearrangement in Non-Small Cell Lung Cancer (NSCLC) in Midwestern Population

J Valentine, M Johnson, Y Zhang, R Raj, R Nayar, K Raparia. Northwestern Memorial Hospital, Chicago, IL

Background: ALK gene rearrangement defines a distinct molecular subtype of non-small-cell lung cancer (NSCLC) that responds to ALK tyrosine-kinase inhibitor crizotinib. Defining distinctive clinical and morphological features of ALK-rearranged lung adenocarcinomas is helpful to identify cases that merit molecular testing.

Design: We screened ALK rearrangements in 280 patients with lung adenocarcinomas diagnosed in 2008 to 2013 at our institution using Vysis dual color break-apart ALK probes. FISH was performed on paraffin embedded tissue sections from cytologic and surgical specimens.

Results: ALK rearrangement was seen in 6 males and 10 females (16/280 or 5.7\%). The median age of patients in our cohort was 54 years (range 29-85 years). All except one of our 16 patients were caucasian. Four patients were smokers, 2 were minimal smokers and 10 were never-smokers. Majority of the patients presented with stage 4 disease (12/16). Mean tumor size was $3 \mathrm{~cm}$ (1 to $6.9 \mathrm{~cm}$, SD 1.9). Only two patients underwent resection of the primary tumor. Most of our patients (13/16) had invasive adenocarcinoma with predominant solid type pattern. The most common cytological features were presence of signet ring (6/16) and pleomorphic (6/16) tumor cells. TTF-1 positivity was seen in 13 of 14 cases. Seven of 16 patients developed multiple brain metastases. The percentage of cells with abnormal FISH signals ranged from $19 \%$ to 93\%. Median follow up duration for our cohort was 15 months (range $0-92$ months). One patient had concurrent KRAS mutation (G12C) in pre-crizotinib specimen. Ten of sixteen patients received crizotinib. Patients with ALK rearrangements receiving crizotinib had a significantly higher survival (median survival 25 months with crizotinib versus median survival 10 months without crizotinib; $\mathrm{p}=0.017, \log$ rank)

Conclusions: Compared to previous studies of patients with ALK gene rearrangements, the patients in our cohort were older and more likely to be caucasian females who were never smokers. The predominant morphology was solid pattern, and the most common cytology was that of signet ring cell. Recognizing patients with ALK-rearranged lung adenocarcinoma who should receive crizotinib resulted in improved survival among our patient cohort, compared with those who received conventional chemotherapy.

2041 The Prognostic Value of Architectural Patterns in a Study of 41 Type AB Thymomas

IT Vladislav, Y Gokmen-Polar, KA Kesler, PJ Loehrer, S Badve. Indiana University School of Medicine, Indianapolis, IN.

Background: Spindle cell thymomas with prominent amounts of lymphocytes are classified as WHO type AB tumors. There are at least two architectural patterns that we have observed in these tumors. One of the patterns was associated with a reticular growth of spindle cells entrapping small islands of tumor cells richly admixed with lymphocytes. We investigated the importance of architectural pattern in type AB thymomas in relation to prognostic value using a series of 41 cases.

Design: Archival hematoxylin and eosin-stained slides from these 41 cases were reviewed for the presence (type 1) or absence (type 2) of reticular growth pattern. Reticular growth pattern was defined as the presence of nests of tumor cells admixed with lymphoid cells and surrounded by bland elongated spindle cells. The architectural patterns were correlated with tumor stage at diagnosis and presence or absence of recurrent disease. 
Results: There was no significant difference in gender distribution or age at presentation in the two subtypes. However, patients with type 1 tumors were more likely to have early stage disease. In contrast, type 2 pattern was associated with higher stage at diagnosis $(P<0.001)$ and greater likelihood for recurrence $(P<0.05)$ and metastases.

Conclusions: Architectural features are prognostically relevant in the classification of WHO AB type thymomas. If the results of this study are confirmed in additional datasets, morphological categorization of $\mathrm{AB}$ tumors may constitute a form of personalized medicine.

2042 The Spectrum of Changes in Adults with Pulmonary Neuroendocrine Cell Hyperplasia and/or Tumorlets: What Are the Minimum Pathologic Criteria to Diagnose DIPNECH?

AE Walts, AM Marchevsky. Cedars-Sinai Medical Center, Los Angeles, CA.

Background: Diffuse idiopathic neuroendocrine cell hyperplasia (DIPNECH) is listed as a preneoplastic condition in the 2004 WHO classification of lung tumors but the diagnostic features can be interpreted variably as the criteria for "diffuse" and the minimum number of tumorlets and/or other findings needed for diagnosis are not specifically defined.

Design: We reviewed 70 consecutive lung wedge biopsies and resection specimens diagnosed with $\mathrm{NECH}$ and/or 2 or more tumorlets at our hospital and classified them into 4 groups: (A) NECH only; (B) tumorlets only; (C) NECH + 2 tumorlets; (D) NECH + 3 or more tumorlets. Within each group we evaluated the incidence of pulmonary carcinoid tumor as evidence for pre-neoplastic potential and the incidence of interstitial fibrosis, bronchiectasis, obliterative bronchiolitis, granulomatous pneumonia, emphysema, and/ or cancer as evidence for a condition associated with reactive rather than "idiopathic" NECH. Results were analyzed with chi-square statistics.

Results: Table I shows the distribution of cases by group and the incidence of carcinoid tumor and/or histological features consistent with reactive rather than idiopathic NECH. The incidences of carcinoid were $72.4 \%, 22.9 \%, 0 \%$ and $0 \%$ in groups $\mathrm{D}, \mathrm{B}, \mathrm{A}$, and $\mathrm{C}$, respectively. The proportion of carcinoid tumors was significantly higher in group $\mathrm{D}$ when compared to other groups $(\mathrm{p}<0.001)$. Within group $\mathrm{D}$, the incidence of reactive features was significantly higher in cases without carcinoid tumor than in cases with carcinoid tumor $(87.5 \%$ vs. $14.3 \%$; $<<0.001)$. Cases in group B showed a low incidence of carcinoid tumors $(22.9 \%)$ and a significantly higher incidence of reactive features $(77.1 \% ; \mathrm{p}<0.0001)$

\begin{tabular}{|c|c|c|c|c|}
\hline & \begin{tabular}{|c|} 
A (NECH only) \\
$N=5$
\end{tabular} & \begin{tabular}{|c|} 
B (tumorlets only) \\
$\mathrm{N}=35$
\end{tabular} & \begin{tabular}{|c}
$\mathrm{C}$ (NECH +2 tumorlets) \\
$\mathrm{N}=1$
\end{tabular} & $\begin{array}{c}\text { D (NECH }+\geq 3 \text { tumorlets) } \\
\mathrm{N}=29\end{array}$ \\
\hline $\begin{array}{l}\text { Carcinoid present } \\
\text { Reactive changes' absent } \\
\text { Reactive changes present }\end{array}$ & $\begin{array}{l}0 \\
0\end{array}$ & $\begin{array}{l}7 \\
1\end{array}$ & $\begin{array}{l}0 \\
0\end{array}$ & $\begin{array}{c}18 \\
3\end{array}$ \\
\hline $\begin{array}{l}\text { Carcinoid absent } \\
\text { Reactive changes absent } \\
\text { Reactive changes present }\end{array}$ & $\begin{array}{l}0 \\
5\end{array}$ & ${ }_{26}^{16}$ & $\begin{array}{l}0 \\
1\end{array}$ & $\begin{array}{l}1 \\
7\end{array}$ \\
\hline
\end{tabular}

- Reactive changes: interstitial fibrosis, bronchiectasis, obititerative bronchiolitis, granulomatous pneumonia, emphysema

Conclusions: Our findings support the use of NECH associated with 3 or more tumorlets and absence of conditions associated with reactive $\mathrm{NECH}$ as minimum diagnostic criteria for DIPNECH. Prospective studies are needed to evaluate the natural history and the incidence of synchronous and metachronous pulmonary carcinoids in patients diagnosed with DIPNECH using these proposed criteria.

2043 Argininosuccinate Synthetase (ASS) Deficiency in High-Grade Pulmonary Neuroendocrine Carcinoma: An Opportunity for Personalized Targeted Therapy

AE Walts, JS Bomalaski, S Orsulic. Cedars-Sinai Medical Center, Los Angeles, CA; Polaris Pharmaceuticals, Inc, San Diego, CA.

Background: Cells that express argininosuccinate synthetase (ASS) can synthesize arginine needed for growth and thus do not rely on absorption of arginine from circulating blood. In contrast, cells deficient in ASS must absorb arginine. Treatment with pegylated arginine deiminase (ADI-PEG 20) selectively eliminates arginine from the circulation and has shown efficacy against ASS-deficient tumors including pulmonary small cell carcinomas (SCC). This study was designed to assess ASS immunostaining in a cohort of high-grade pulmonary neuroendocrine carcinomas (PNEC)

Design: 69 PNEC (49 SCC and 20 large cell NEC; LCNEC) diagnosed by current WHO criteria were retrieved from our files. Formalin fixed paraffin embedded sections of the 54 primary tumors, 15 metastases, and controls were stained using an ASS-specific monoclonal antibody (Polaris Pharmaceuticals, Inc. San Diego, CA). Benign bronchial and alveolar lining cells served as internal positive controls. Tumors exhibiting positive staining of any intensity were recorded as ASS-positive. Positive staining in $<30 \%$ of the tumor was scored as weak. Staining in $\geq 30 \%$ of the tumor was scored as strong. Absence of staining was recorded as ASS-negative.

Results: The patients ( 34 males, 35 females) ranged from 45 to 85 years in age (median $68 \mathrm{yrs}$ ) at surgery. The primary tumors included biopsies and resections; the metastases were from regional lymph nodes and various distant sites. $40(58 \%)$ of the PNEC including $30(61.2 \%)$ SCC and $10(50 \%)$ LCNEC were ASS-negative. These ASS-negative tumors included $34(63.0 \%)$ primary and $6(40 \%)$ metastatic lesions. Weak staining was seen in $19(27.5 \%)$ cases including $14(28.6 \%)$ SCC and $5(25 \%)$ LCNEC. Strong staining was seen in only $10(14.5 \%)$ cases including $5(10.2 \%)$ SCC and $5(25 \%)$ LCNEC.

Conclusions: More than $50 \%$ of the high-grade pulmonary neuroendocrine carcinomas (PNEC) tested lack immunohistochemically detectable ASS suggesting they are auxotrophic for arginine and could benefit from arginine deprivation therapy.
Immunostaining for ASS has potential to improve selection of patients with PNEC for arginine-deprivation therapy with ADI-PEG 20.

2044 Clonality Analysis of Pulmonaly Mucocicliated Glandular Tumors

M Watanabe, T Yokose, C Hasegawa, S Matukuma, Y Miyagi. Kanagawa Cancer Center Hospital, Yokohama, Kanagawa, Japan; Kanagawa Cancer Center Research Institute, Yokohama, Kanagawa, Japan.

Background: Although peripheral pulmonary lesions including ciliated cells are deemed non-neoplastic, some were reported as neoplastic and termed as mucociliated papillary tumors and very well differentiated adenocarcinomas. We examined 6 ciliated lesions for 14 years and have reported cases of pulmonary mucociliated glandular tumors (PMGT); however, the lesion neoplasticity was not identified. We developed a new method for clonality analysis using the human androgen receptor (HUMARA) that identifies if a lesion is a neoplasia or not. Here, we report the results of PMGT.

Design: All resected PMGT specimens were obtained from 2 male and 4 female patients who underwent lesion resection at the Kanagawa Cancer Center Hospital from 2000 to 2013. A well-defined round or oval mass in the peripheral lung parenchyma was a representative microscopic feature of PMGT. Stratified epithelial cells lining the surfaces of the bronchioles and alveoli grow papillary and include ciliated and goblet cells. Mucin often fills the airspaces. We conducted clonal analysis using an improved method for HUMARA gene amplification. Its strategy involves random X chromosome inactivation by methylation (lyonization) and methylated gene-specific polymerase chain reaction amplification after sample DNA digestion with the methylation-sensitive restriction enzyme, HpaII. This method is used only for female patients. Further, identifying small differences in the number of CAG trinucleotide repeats in the gene is difficult using the conventional method; therefore, $<50 \%$ of female patients were available for the clonality analysis. We established an improved HUMARA assay combined with a DNA heteroduplex assay called the loop-hybrid mobility shift assay, which could identify 1 CAG repeat number difference; this increased the rate of informative female patients to $70 \%$.

Results: We analyzed 2 out of 4 female patients. One case was not informative. Another case was divided into 4 areas and analyzed. Three areas had a monoclonal pattern. However, we noted a different clonality pattern between 1 and 2 areas in 1 tumor and a monoclonal pattern in 1 area. We reviewed the histology again, but no histopathological differences were found between these 4 areas.

Conclusions: Our results suggest that this tumor was a non-neoplastic lesion; however, the possibility of neoplasm cannot be denied completely because some areas had a monoclonal pattern. Further studies with more cases are needed to clarify the presence or absence of clonality in PMGT.

2045 Primary Mediastinal Seminomas: A Comprehensive Immunohistochemical Study Using Novel Markers

A Weissferdt, NKalhor, H Liu, J Rodriguez, J Fujimoto, X Tang, II Wistuba, CA Moran. MD Anderson Cancer Center, Houston, TX.

Background: Primary mediastinal seminomas are unusual tumors that can present in a pure form or as a component of a mixed germ cell tumor. Whereas numerous studies have been performed to characterize the immunohistochemical phenotype of testicular seminomas using novel antibodies, relatively little is known about the expression of such markers in primary mediastinal seminomas. This study investigates the immunohistochemical features of these tumors using a comprehensive panel of novel markers in this context.

Design: Thirty-two cases of pure primary mediastinal seminomas were reviewed and representative whole tissue sections were selected for immunohistochemical studies using antibodies directed against high molecular weight cytokeratin (CK5/6), low molecular weight cytokeratin (CAM5.2), OCT3/4, SALL4, GATA3, SOX2, SOX17, TCL1, glypican3, MAGEC2, and Pax8. The percentage of positive tumor cells as well as the intensity of staining were evaluated and scored.

Results: Thirty-one cases (97\%) of primary mediastinal seminoma expressed SOX17 while 29 cases $(91 \%)$ were positive for OCT3/4 and SALL4, respectively . Twentyeight cases (88\%) expressed MAGEC2 and CAM5.2 (perinuclear dot-like pattern), respectively. Two cases $(6 \%)$ were positive for Pax 8 and a single case $(3 \%)$ was positive for TCL1. None of the cases stained with CK5/6, GATA3, SOX2, and glypican3. Conclusions: Similar to their testicular counterparts, primary mediastinal seminomas show consistent expression of OCT3/4, SALL4. SOX17 and MAGEC2 and are negative for SOX2, glypican3, GATA3 and CK5/6. In a similar manner, Pax 8 positivity is only inconsistently identified in mediastinal seminomas. Contrary to testicular seminomas, however, mediastinal tumors show diffuse expression of low molecular weight cytokeratin in up to $90 \%$ of cases and are commonly negative for TCL1. Although there is some immunohistochemical overlap between testicular and mediastinal seminomas, differences also exist and need to be taken into account when dealing with these tumors.

2046 Mediastinal Seminomas with Florid Follicular Hyperplasia: A Clinicopathological and Immunohistochemical Study of 6 Cases

A Weissferdt, CA Moran. MD Anderson Cancer Center, Houston, TX.

Background: Thymic seminomas are unusual primary tumors of the anterior mediastinum. Morphologically they are usually indistinguishable from their testicular counterparts, however, in the mediastinum, the occurrence of cystic changes in association with seminoma is not uncommon. During a recent review of thymic seminomas, 6 cases associated with florid follicular hyperplasia were identified the details of which are described herein.

Design: During a recent review of 45 mediastinal seminomas, 6 cases with florid follicular hyperplasia were identified and clinical information including presentation, treatment and follow up was analyzed. In order to characterize the lymphoid component, 
representative whole tissue sections were selected for immunohistochemical studies using antibodies directed against S-100, CD1a, fascin, CD3, CD4, CD8, CD20, CD99, kappa and lambda. The distribution of the lymphoid elements as well as clonality were assessed and recorded.

Results: The patients were male with an age range from 21 to 33 years. Symptoms included cough, dyspnea and chest pain. All patients underwent thymectomy. Macroscopically, all tumors had a fleshy, lobulated cut surface. Histologically, the tumors were composed of round to polygonal cells with indistinct cell borders, clear cytoplasm and prominent nucleoli. All tumors were accompanied by small lymphocytes infiltrating fibrovascular septa and 3 showed granuloma formation. Striking follicular hyperplasia was evident in all cases, with tumor interspersed between prominent follicles with germinal centers. While these germinal centers were largely composed of mature B lymphocytes, mature $T$ lymphocytes were the predominant cell component of the interfollicular areas. Immature T cells were not identified. Scattered Langerhans cells were noted in between tumor cells and dendritic cells were seen in the mantle zones. All cases failed to show light chain restriction. Clinically, 3 patient received adjuvant chemotherapy. Follow-up showed that all patients were alive 12 to 48 months after diagnosis.

Conclusions: In addition to cystic changes, thymic seminomas can be associated with striking lymphoid follicular hyperplasia to a degree whereby the tumor may be masked by the lymphoid component. The lymphoid infiltrate is composed of a mix of mature B and T cells of a polyclonal nature. Familiarity with the possibility of thymic seminoma associated with florid follicular hyperplasia is important in order not to misdiagnose these cases for pure thymic follicular hyperplasia, which would require a different treatment approach.

\section{Paraganglioma Versus Thymic Carcinoid Tumor: An Immunohistochemical Study of 46 Cases}

A Weissferdt, N Kalhor, H Liu, J Rodriguez, J Fujimoto, X Tang, II Wistuba, CA Moran. MD Anderson Cancer Center, Houston, TX.

Background: Paragangliomas and primary carcinoid tumors of the mediastinum are lesions that belong to the neuroendocrine family of neoplasms. These tumors can share significant histological and immunohistochemical overlap. Correct diagnosis, however, is of utmost importance as paragangliomas most often run a benign clinical course whereas primary carcinoid tumors of the mediastinum exhibit more aggressive biologic behavior. To this end, we performed a comparative immunohistochemical analysis using traditional and novel markers in 22 cases of paraganglioma and 24 cases of primary mediastinal carcinoid tumors.

Design: Twenty-two cases of paraganglioma and 24 cases of primary mediastinal carcinoid tumors were reviewed and representative whole tissue sections were selected for immunohistochemical studies using antibodies directed against pancytokeratin (OSCAR), thyroid transcription factor-1 (TTF-1), napsin A, chromogranin A, synaptophysin and GATA-3. The percentage of positive tumor cells as well as the intensity of staining were evaluated and scored and the results compared between the two tumor types.

Results: All cases of paraganglioma were positive for chromogranin A and synaptophysin and negative for pancytokeratin, TTF-1 and napsin A. GATA-3 showed expression in $12 / 22$ tumors $(55 \%)$. Contrary to that, the carcinoid tumors were uniformely positive for pancytokeratin, and negative for napsin A and GATA-3. Synaptophysin and chromogranin A were expressed in $79 \%$ of cases, respectively. TTF-1 was positive in $4 / 24$ of the carcinoid tumors ( $17 \%)$.

Conclusions: Although pancytokeratin is confirmed to be the single most useful marker in the separation of paragangliomas from primary mediastinal carcinoid tumors, two other markers are shown to be useful antibodies in this context. GATA-3, a novel marker commonly used in the diagnosis of breast and urothelial cell carcinomas, was shown to be expressed in $55 \%$ of paragangliomas but none of the carcinoid tumors. Reversely, TTF-1 can be positive in up to $17 \%$ of primary mediastinal carcinoid tumors but was not identified in any of the paragangliomas.

\section{8 c-Met Immunohistochemistry in EGFR Mutant Adenocarcinoma:} Comparison with Fluorescence In-Situ Hybridization

BC Willis, DR Martin, D Saxe, SS Ramalingam, F Khuri, KE Fisher, MR Rossi, C Cohen, G Sica. Emory University, Atlanta, GA; Winship Cancer Center, Atlanta, GA. Background: Targeted therapy of activating EGFR mutations in lung adenocarcinomas with tyrosine kinase inhibitors (TKI) results in decreased tumor burden that is often a transient response due to the presumed outgrowth of TKI resistant clones. c-Met amplification accounts for $5-20 \%$ of EGFR TKI resistance. Cytogenetic methods are traditionally used to detect c-Met amplification. However, immunohistochemistry (IHC) offers a cost-effective alternative or adjunctive method for detection. We compared c-Met IHC to fluorescence in-situ hybridization (FISH) as the gold standard. Design: Five micron sections of 18 cases with c-Met FISH assays were immunostained for c-Met monoclonal clone SP44 (1:100) (Spring BioScience, Pleasanton, CA). Results of expression and amplification were compared.

Results: In 18 adenocarcinoma cases, c-Met IHC identified $100 \%$ of c-Met FISH amplified tumors with $3+$ staining in greater than $80 \%$ of tumor cells $(n=2) .6$ cases showed 2-3+ positive IHC but no amplification was detected by FISH. 10 cases were negative for both IHC and FISH. False positives observed with IHC occurred only in cytologic specimens $(n=6)$. One case produced positive c-Met IHC and negative FISH in a cytologic specimen, but was negative by IHC and FISH in the resection specimen of the same lesion.

\begin{tabular}{|l|l|l|l|}
\hline & FLSH Positive & FISH Ncgative & \\
\hline IHC Positive (8) & 2 & 6 & PPV. 0.25 \\
\hline IHC Neqative (10) & 0 & 10 & NPV.1 \\
\hline & Sensitrity- $100 \%$ & Spscificity- $63 \%$ & \\
\hline
\end{tabular}

Conclusions: c-Met IHC is a quick, economical and sensitive screening test for c-Met expression. Significant false positives limit the tests specificity when compared to FISH as gold standard. Differences in fixation techniques in cytologic preparations may account for some false positive cases. In summary, IHC screening with subsequent FISH of positive cases should exclude false positives and limit costs for this infrequently amplified gene.

\section{Disparities in Non-Small Cell Lung Cancer Outcomes}

F Yamani, W Zhao, R-A Monde, I Reis, J Hu, B Lally. University of Miami Miller School of Medicine/Jackson Memorial Hospital, Miami, FL.

Background: Racial and ethnic disparities in non-small cell lung cancer (NSCLC) have been reported; the significance is the import given the increasing diversity of the US population. Identifying the etiologies leading to NSCLC disparities would improve the quality of life for millions of Americans, reduce health care costs, and improve health care delivery on a national scale. We investigated NSCLC disparities in a tri-racial/ ethnic population with a high proportion of underserved minorities.

Design: We used a retrospective study design to retrieve clinical data on NSCLC patients (AJCC stages I-III) who were diagnosed and treated during 2000-2010 at two medical centers. Selection criteria included race/ethnicity of African American (AA), Hispanic White (HW), and non-Hispanic White (NHW) with confirmed pathology records. Differences of patient characteristics/pathology/survival among AA/HW/NHW were analyzed by Chi-square test. Overall survival (OS) was analyzed using Kaplan-Meier, log-rank test and Cox's proportional hazard regression.

Results: 845 patients (118 AA, $269 \mathrm{HW}, 458 \mathrm{NHW}$ ) included in this analysis have an overall median survival of 4 years $(y r)$. There was racial/ethnic difference $(p<0.001)$ in OS rates at 4 years: $37.8 \%$ for AA, $37.2 \%$ for HW, and $60.3 \%$ for NHW. A larger percentage of the AA $(71.2 \%)$ and the HW $(61.3 \%)$ were males; the NHW group had more females $(55.7 \%)(\mathrm{p}<0.001)$. The mean age for AA $(60.9 \mathrm{yr})$ and HW $(61.6 \mathrm{yr})$ was younger than for NHW $(67.9 \mathrm{yr})(\mathrm{p}<0.001)$. AA and HW had more advanced stage disease $(\mathrm{p}<0.001)$ and experienced a different profile of NSCLC histologies $(\mathrm{p}<0.001)$. The utilization rate of both surgery $(\mathrm{AA}=46.6 \%, \mathrm{HW}=52.8 \%$, and $\mathrm{NHW}=84.1 \%$; $\mathrm{p}<0.001)$ and radiotherapy $(\mathrm{AA}=25.4 \%, \mathrm{HW}=32.3 \%, \mathrm{NHW}=14.8 \% ; \mathrm{p}<0.001)$ showed significant difference $(\mathrm{p}<0.001)$. The racial/ethnic difference in OS was significant in univariate analysis. In multivariate analysis, worse OS was significantly associated with increasing age, male gender, increasing stage, adenocarcinoma histology (vs. bronchioloalveolar carcinoma as the referent group), and non-surgical-based therapy. Conclusions: In this population, underserved minorities have worse OS. The contributing factors are likely multifocal in nature. We plan to further characterize NSCLC disparities within this cohort by investing smoking history/status, comorbidities, health care delivery, and somatic mutations.

2050 Increased Epithelial-to-Mesenchymal Transition in Non-Small Cell Lung Cancers of Diabetic Patients

$X$ Yang, H Mani, D Zander, G Clawson, J Varlotto, N Rassaei. PSHMC, Hershey, PA; UMSS, Worcester, MA.

Background: Early-stage non-small cell lung carcinoma (NSCLC) is primarily treated with surgery. Local recurrence of resected NSCLC has been reported to be more frequent in diabetics in our patient cohort. Epithelial-to-mesenchymal transition (EMT) plays a key role in local tumor recurrence and metastasis. Acquisition of mesenchymal phenotype during EMT results in increased expression of vimentin. Further, the tumor suppressor HtrA1 has recently been identified as a major regulator of EMT, reportedly playing a role in lung cancer metastasis. HtrAl regulates the availability of insulinlike growth factors (IGFs) by cleaving IGF-binding proteins. We investigated whether diabetes is associated with alteration in expression of HtrA1, IGF-1 receptor (IGF-1R), and vimentin in our cohort of NSCLC patients.

Design: 40 resected early-stage NSCLCs [20 adenocarcinomas (ADCs) and 20 squamous carcinomas (SqCCs); 10 of each from diabetic and non-diabetic patients] were included for study. Immunohistochemistry (IHC) was performed for vimentin, HtrA1, and IGF-1R. Extent of staining was scored as 0 (no staining), $1+(<25 \%$ positive), $2+$ $(25-49 \%$ positive $), 3+(50-75 \%$ positive $)$ and $4+(>75 \%$ positive $)$. Staining intensity was scored as 0 (negative), 1 (mild), 2 (moderate) and 3 (intense). Vimentin staining was scored in stromal cells; HtrA1 and IGF-1 staining were scored in tumor cells. An IHC score was calculated by multiplying extent of staining with intensity. Mean IHC scores between diabetic and non-diabetic groups were compared by t-test using SigmaPlot. Results: NSCLCs in diabetic patients showed significantly lower expression of HtrA1 $(0.22$ vs. $2.00, p<0.05)$ and higher expression of vimentin $(4.74$ vs. $2.05, p<0.05)$, suggesting a greater degree of EMT. Tumors in diabetics also showed greater degree of IGF-1R expression ( 1.59 vs. $0.58, \mathrm{p}<0.05)$. This significance was maintained in both ADCs and SqCCs for HtrAl and vimentin. Mean IHC scores in ADCs of diabetics vs. non-diabetics were 0.40 vs 2.45 (HtrA1) and 4.36 vs. 2.2 (vimentin). Mean IHC scores in SqCC of diabetics vs. non-diabetics were 0 vs. 1.5 (HtrA1) and 5.25 vs 1.91 (vimentin). Although IGF-1R expression was higher in diabetics in both SqCCs (1.75 vs 0.50$)$ and ADCs (1.44 vs. 0.75), it attained statistical significance only for SqCC. Conclusions: Our pilot data suggests that the biology of EMT in NSCLCs is different in diabetics than in patients without diabetes. Suppression of EMT regulator HtrA1 and exaggerated EMT in tumors of diabetics may be responsible for the increased local recurrence seen in diabetics. 
2051 Synchronous Lung Adenocarcinoma and Primary Pulmonary MALT Lymphoma: An Underdiagnosed Entity Associated with KRAS Mutations $J$ Yao, NRehktman, K Nafa, A Dogan, M Ladanyi, ME Arcila. Memorial Sloan-Kettering Cancer Center, New York, NY.

Background: Pulmonary extranodal marginal zone lymphoma (MZL / MALT) is a rare entity accounting for less than $0.5 \%$ of primary pulmonary malignancies. The occurrence of lung adenocarcinoma (AD) and primary pulmonary MALT lymphoma as collision tumors have only been rarely reported. We investigated the concurrent incidence of these two entities in a large cohort of lung $\mathrm{AD}$ cases submitted for routine molecular diagnostic testing.

Design: Consecutive lung AD cases were reviewed and categorized based on the presence or absence of a chronic inflammatory lymphoid infiltrate and scored as 0 absent/minimal infiltration, 1+ moderate or 2+ intense. Results of molecular testing for recurrent mutations in EGFR, KRAS, BRAF, HER2, PIK3CA, AKT, MEK1 and fusions involving ALK were recorded. IgH rearrangement studies and immunohistochemical stains for CD3, CD20, Kappa and lambda were performed in cases with intense (2+) lymphoid infiltrates.

Results: A total of 600 lung adenocarcinoma cases were reviewed. None of the patients had prior history of a lymphoproliferative disorder. Lung adenocarcinoma driver mutations were identified in 389 cases $(65 \%)$. In $319(53 \%)$ tumors the lymphoid infiltrate was scored as $0,264(44 \%)$ as $1+$ and $17(3 \%)$ as $2+$. A score of $2+$ was most commonly associated with a KRAS mutation $(10 / 17,59 \%, \mathrm{p}=0.03)$. Among the cases with intense lymphoid infiltrates, 4 (24\%) had clonal IgH rearrangement as well as morphologic and immunophenotypic features consistent with MALT lymphoma (including a nodular distribution, predominance of CD20+ B cells and light chain restriction). In all cases, the $\mathrm{AD}$ was intimately associated with the lymphoma component and there was no clinical evidence of a systemic lymphoproliferative disorder. A KRAS mutation was identified in 3 cases, 1 case was wild type. The incidence of MALT lymphoma associated with lung $\mathrm{AD}$ is $0.7 \%$ in this series.

Conclusions: The synchronous occurrence of lung $\mathrm{AD}$ with an intimately associated MALT lymphoma is higher than previously reported. The distinction between an exuberant reactive lymphocytic infiltration and a MALT lymphoma, in the setting of lung $\mathrm{AD}$, can be challenging and may be often underdiagnosed if not fully investigated. While the etiology is uncertain, the presence of a KRAS mutation in 3 out of the 4 synchronous lesions is an interesting finding which warrants further investigation in terms of its implications for tumor immunology and newer immunotherapy approaches.

\section{Quality Assurance}

2052 Factors That Determine Patient Consent for Research BioBanking and BioSpecimen Research: Lessons for Optimizing Specimen-Driven Pathology Research

A Alam, A Ali, N Amin, D Chadwick, MHA Roehrl. University Health Network, University of Toronto, Toronto, ON, Canada.

Background: With rapidly increasing interest in understanding, measuring, and optimizing the patient consent process for research biobanking, the BioSpecimen Sciences Program (BSP) set out to study its current research consent process. We attempted to establish comprehensive baseline consent rates for all disease site groups in a multi-campus academic health system. We set out to discover any variability among disease site groups as well as to determine whether consents obtained through direct patient encounters of BSP staff were superior over traditional consent mechanisms.

Design: During a one-year period, all surgical patients were reviewed for individual research consent status as one of the four categories: 'Signed' (acceptance or refusal), 'Not signed' (no declaration), 'No consent' (no form in clinical chart), or 'No chart' (chart unavailable). Patients were further divided according to clinical subspecialty group. In addition, daily pre-admission schedules were screened for patients who were eligible for specific institutional research studies, and BSP coordinators approached these patients in person.

Results: 4007 patients had research consent forms that could be evaluated. Of these, $2740(68.4 \%)$ consents were signed. $2730(68.1 \%)$ patients agreed to biobanking for research while only $10(0.2 \%)$ refused explicitly. The highest response rates of consent came from patients seen by the genitourinary $(91.6 \%)$ and gynecological site groups $(91.4 \%)$, whereas dermatology $(45.5 \%)$ and plastic surgery $(9.2 \%)$ were lowest. 246 patients were approached in person by BSP coordinators. This produced a response rate of $98.8 \%$ with 234 patients $(95.1 \%)$ agreeing to biobanking, 8 (3.3\%) refusing, 1 $(0.4 \%)$ withdrawing, and $3(1.2 \%)$ undecided. BSP staff increased the number of patients who agreed to biobanking rom 116 (previously consented by non-BSP staff) to 234 . Conclusions: The surgical preadmission package is an effective mechanism for research consenting. However, there was significant variability between different clinical site groups (9.2-91.6\%). Reasons include staff buy-in, consent logistics, competing research consents, and heterogeneity of research volume and clinical trial activities. Pathology/ BSP research coordinators are highly effective in optimizing research consent rates (98.8\% response, $95.1 \%$ acceptance). Personal involvement of BSP staff more than doubled enrollment of our "highest-value" patients. Personal involvement of both pathologists and biobank staff is key for achieving optimal consent rates.

2053 Temperature-Based Antigenicity Preservation Methods for Tissue Microarrays: Longitudinal Prospective Study

NK Andeen, JM Brooks, $R$ Bowman, T Baullinger, MS Tretiakova. University of Washington, Seattle, WA.

Background: Studies have shown time-dependent loss of antigenicity for a variety of diagnostic, prognostic and predictive antibodies used on precut paraffin slides. However, there are no established guidelines for storage methods of precut controls, patient tissue, or microarrays (TMAs). We sought to determine loss of antigenicity and potential for preservation by refrigeration in a longitudinal prospective study.

Design: Selected diagnostic or prognostic antibodies included p53, IDH-1, Ki67, synaptophysin, and androgen receptor (AR). TMA with 22 cores from small cell carcinomas, prostatic adenocarcinomas, and gliomas was constructed; 125 slides were cut at 4 microns at time 0 . Slides were stored exposed to air at room temperature (RT), $4 \mathrm{C}$, or -20C; IHC was performed on the Leica Bond III at time 0 , weeks $1,2,4$, and 6 . Each tissue core was scored for overall intensity ( 0 to $3+)$ and \% of cells staining (100 cells within hotspot). Loss of antigenicity was defined as a decrease of staining intensity by one order and/or loss of $\geq 10 \%$ of positive cells compared to time 0 .

Results: By 6 weeks, Ki67, p53 and AR antibodies had progressive loss of detectable immunoreactivity, which was most prominent for those stored at RT $(\mathrm{p}<0.05)$. For Ki67, significant loss of antigenicity was first detected at 1 week on slides stored at RT; storage at $-20 \mathrm{C}$ preserved $\%$ positive cells until 4 weeks; at 6 weeks, storage at all 3 temperatures had loss of expression. p53 had sustained loss of antigenicity at 4 weeks $(\mathrm{p}<0.05)$ in slides stored at RT and 4C, which manifested as decreased intensity rather than $\%$ of reactive cells. AR had loss of $\%$ staining at 6 weeks at RT only. Synaptophysin and IDH-1 had no loss of reactivity regardless of storage temperatures.

Loss of $\geq 10 \%$ cells staining positively in hotspots, and/or loss of intensity of staining for Ki67 (A) and p53 (B), * $(p<0.05)$
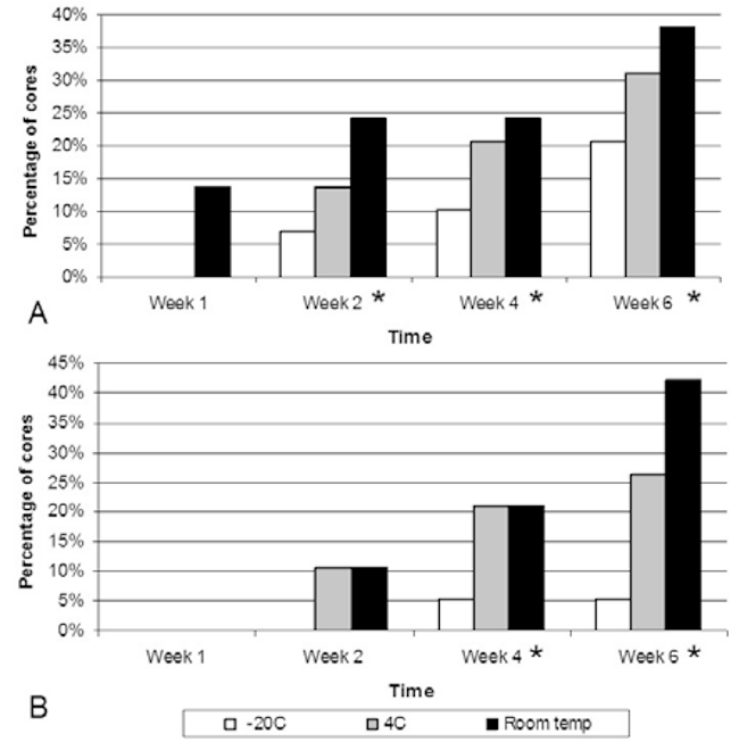

Conclusions: Loss of antigenicity is antibody dependent and most notable for slides stored at RT. Refrigeration of slides significantly delayed loss of antigenicity for all affected antibodies, especially at $-20 \mathrm{C}$. Progressive decrease in Ki67 and p53 $\%$ positivity and staining intensity could affect reporting of prognostic results; thus refrigeration should be considered for long-term storage. Monitoring of antigenicity loss at longer time-periods as an ongoing part of the study will define optimal storage recommendations.

2054 Flocculant Artifact Caused by Sterile Lubricant in Fine Needle Aspirates (FNAs)

KJ Brister, JM Paulson, EB O'Neill, DW Cohen, JV Hennessey. Beth Israel Deaconess Medical Center, Boston, MA; Tampa General Hospital, Tampa, FL; Ohio State University College of Medicine, Columbus, $\mathrm{OH}$.

Background: The use of sterile lubricant in place of conducting gel while performing ultrasound guided FNA of the thyroid is common practice. As a result of a new purchasing policy at our institution, our usual sterile lubricant (Surgilube ${ }^{\circledR}$ ) was replaced with an alternative gel (Allegiance $(\mathbb{R})$. Soon thereafter, reports of non-diagnostic thyroid FNA cytology results due to obscuring artifacts became evident. The purpose of this study is to test three brands of lubricating gel to determine their propensities for causing artifacts in FNA specimens.

Design: A pannus, removed for obesity, was identified. Four $3 \times 3 \mathrm{~cm}$ areas, labeled A,

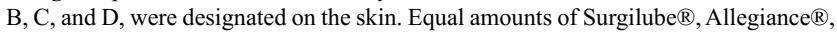
and E-Z Lubricating Jelly ${ }^{\circledR}$ were applied to areas A, B, and C, respectively. No gel was applied to area D. Three containers of CytoLyt ${ }^{\circledR}$ were designated for each area. A 25 gauge needle was used to aspirate the pannus through the gel, one needle per pass. Three passes were rinsed into each container. In total, 12 ThinPrep ${ }^{\circledR}$ slides were prepared. The randomized and de-identified slides were evaluated by a cytopathologist for amount of flocculant artifact $(0=$ none, $1=$ mild, $2=$ severe $)$ and number of tissue fragments. 